\title{
Synaptic Physiology of the Cochlear Sound Encoding
}

\author{
Dissertation \\ for the award of the degree \\ "Dr. rerum naturalium" (Dr. rer. nat.) \\ Division of Mathematics and Natural Sciences \\ of the Georg-August-Universität Göttingen \\ submitted by \\ Tzu-Lun Wang \\ from Kaohsiung, Taiwan
}

Göttingen, 2012 
Members of the thesis committee:

Prof. Dr. Tobias Moser

InnerEarLab, Dept. of Otolaryngology, UMG

Prof. Dr. Martin Göpfert

Dept. of Cellular Neurobiology, Johann-Friedrich-Blumenbach-Institute for Zoology und Anthropology

Prof. Dr. Fred Wolf

Dept. of Nonlinear Dynamics, Max-Planck-Institute for Dynamics and Self-Organization

Date of the oral examination: 


\section{Declaration}

This thesis has been written independently and with no other sources and aids than quoted.

Tzu-Lun Wang

Göttingen, $19^{\text {th }}$ of December, 2012 


\section{Content}

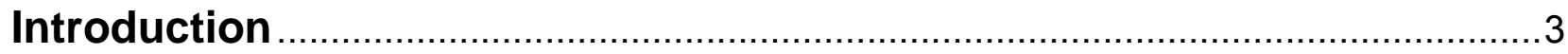

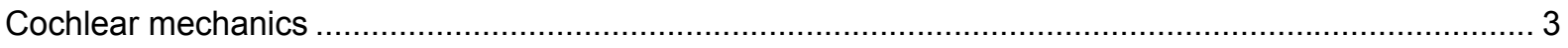

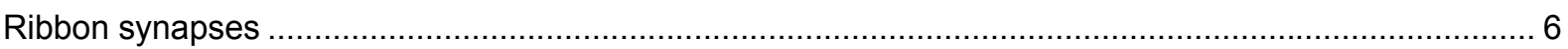

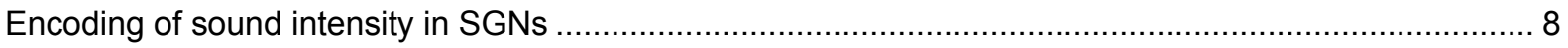

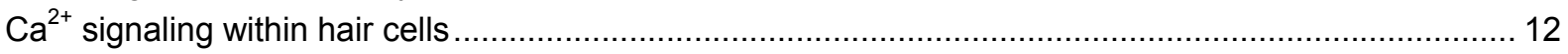

Aims of this work

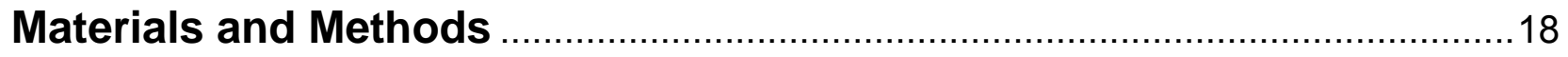

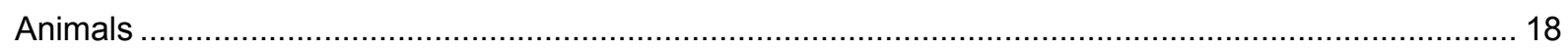

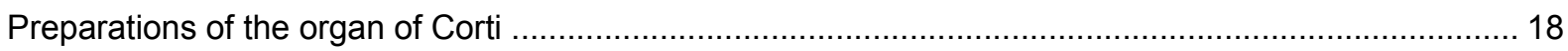

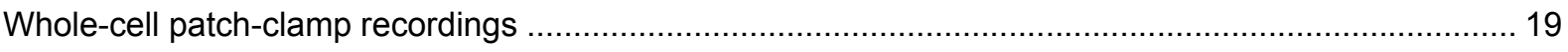

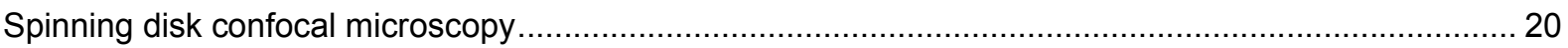

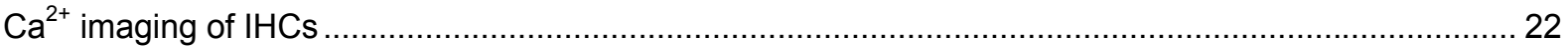

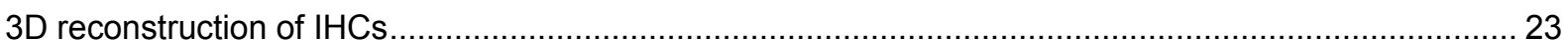

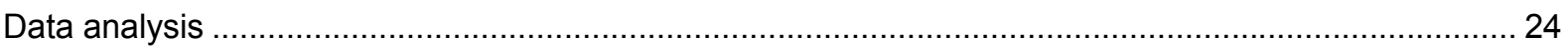

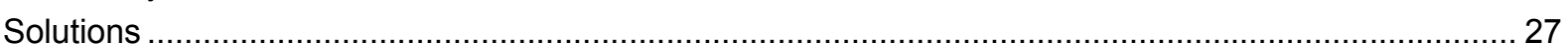

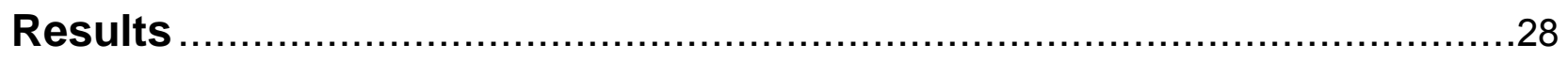

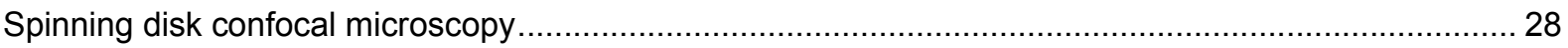

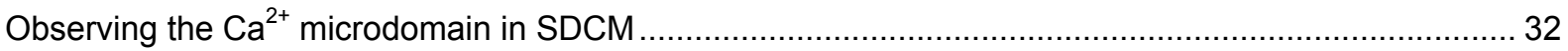

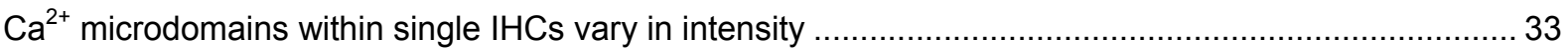

Voltage dependence of activation varies among different $\mathrm{Ca}^{2+}$ microdomains ....................................... 39

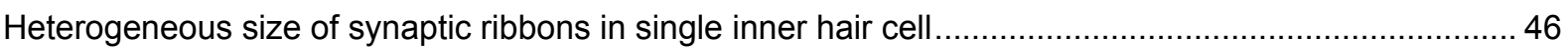

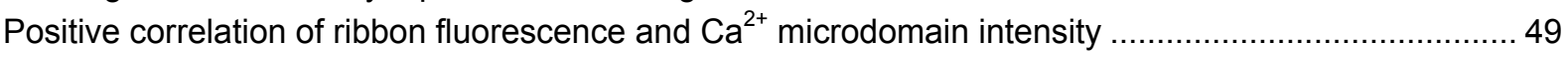

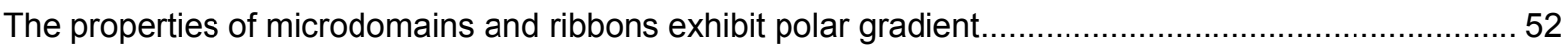

Longitudinal properties of microdomains and ribbons do not show differences.................................... 60

Discussion

Heterogeneity of presynaptic $\mathrm{Ca}^{2+}$ signals within and across IHCs 
Mechanisms for the heterogeneity of voltage-dependent activation of $\mathrm{Ca}^{2+}$ microdomains

The sizes of synaptic ribbons correlate with the intensities of $\mathrm{Ca}^{2+}$ microdomains...................................68

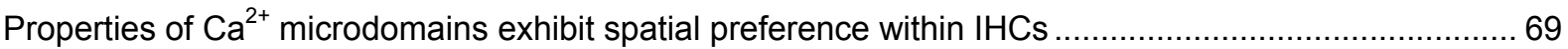

Presynaptic and postsynaptic mechanisms of diverse spontaneous rate in SGNs ............................... 71

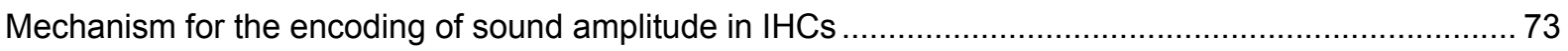

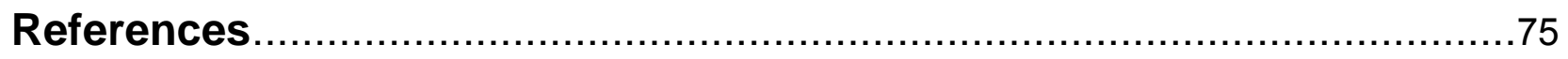

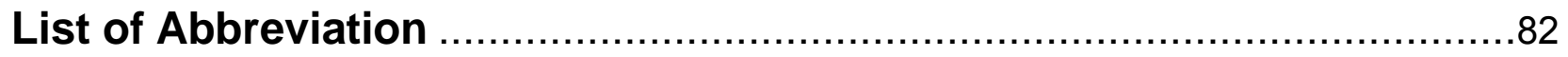

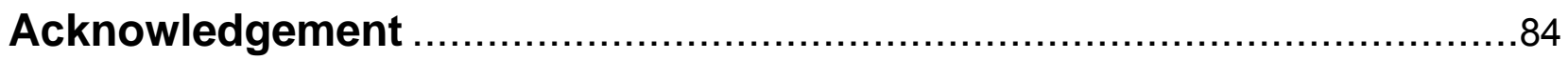

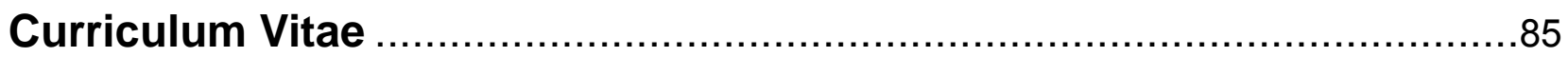




\section{Abstract}

Sound intensity is encoded as action potential firing rates by spiral ganglion neurons. These neurons show different rate-level functions with different auditory thresholds, and are thought to collectively encode the large dynamic range of the auditory stimuli. Hair cell ribbon synapses drive the action potential firing in spiral ganglion neurons through release of glutamate that is triggered by voltage-gated $\mathrm{Ca}^{2+}$ channels. Therefore, the investigation of presynaptic $\mathrm{Ca}^{2+}$ signaling and its relationship to synaptic ribbons might help to explain how inner hair cells (IHCs) decompose auditory information at their heterogeneous ribbon synapses thereby driving neurons with different rate-level functions.

In this study, patch-clamp and $\mathrm{Ca}^{2+}$ imaging by spinning disk confocal microscopy were performed to investigate the presynaptic $\mathrm{Ca}^{2+}$ signaling at all active zones of a given IHC. Presynaptic $\mathrm{Ca}^{2+}$ microdomains and ribbons varied in their properties within and among cells. Moreover, their fluorescence intensities were positively correlated. Additionally, we assessed the spatial distribution of the properties of synaptic ribbon and $\mathrm{Ca}^{2+}$ microdomain relative to the circumferential position of the inner hair cell. Larger ribbons and low voltage-sensitivity $\mathrm{Ca}^{2+}$ microdomain exhibit the preference to localize to the modiolar (neural) side.

Our results imply that larger synaptic ribbons are associated with more $\mathrm{Ca}^{2+}$ channels, which is expected to enhance neurotransmitter release at those synapses. Interestingly, previous studies (Merchan-Perez \& Liberman, 1996) on cat cochlea suggested that high spontaneous rate auditory nerve fibers mainly innervate the pillar (abneural) face of inner hair cells. If conserved among species, our finding of high voltage-sensitivity $\mathrm{Ca}^{2+}$ microdomains on the 
pillar face seems support the high spontaneous-rate neuron innervating this face. Therefore, the hair cell ribbon synapses might utilize different voltage-sensitivities of their $\mathrm{Ca}^{2+}$ channels to determine the sound coding by the postsynaptic spiral ganglion neurons. 


\section{Introduction}

\section{Cochlear mechanics}

The mammalian cochlea is a very elaborate organ containing several active mechanisms for processing the incoming sensory stimuli conferring it with sensitivity. The ear can process acoustic stimuli that largely range in frequency and amplitude. The human cochlea, for example, can identify sound frequencies from 0.1 to $20 \mathrm{kHz}$ (Fettiplace and Hackney, 2006) and, surprisingly, distinguish between 2 tones with only a $0.5 \%$ difference of frequency (Hudspeth, 2008). Likewise, mammals can encode sounds with six orders of difference, which have a trillion-fold difference in energy (Hudspeth, 2008).

Several ingenious mechanical designs are utilized in the cochlea to accomplish these tasks. The organ of Corti is the core structure for sound processing in the cochlea. In the organ of Corti, the sensory cells, inner (IHCs) and outer (OHCs) hair cells are sandwiched between two accellular membranes, i.e., the basilar and tectorial membranes, whereby the stereocilia of OHCs are attached to the tectorial membrane. The propagating sound wave in the perilymph of the scala tympani vibrates the basilar membrane, thereby leading to relative movements of the tectorial membrane and the hair cells resulting in the deflection of the stereocilia of IHCs and OHCs.

The micromechanical properties of the cochlea enable a decomposition of the frequency components of an acoustic stimulus along the basilar membrane, which is narrow and stiff at the base and wide and soft at the apex of the cochlea. These anatomical characteristics determine the inertia $(\mathrm{M})$ and stiffness $(\kappa)$ of different cochlea partitions thereby defining the impedance of them (Geisler, 1998; Hudspeth, 2008). The characteristic frequency (CF) $\omega_{0}$ which equals the $\sqrt{ }(\kappa / M)$ of the specific partition manifest the lowest impedance for this 
partition and results in the resonance of the basilar membrane, thereby amplifying the intensity of this CF. The tonotopic map of the cochlea is established according to the increase of $\kappa$ and decrease of $M$ along the cochlear axis from base to apex.

In addition to the basilar membrane, the active movement of bundles of stereocilia and the somatic electromotility of OHCs furthermore amplify the vibration synergistically. The stereocilia of both $\mathrm{IHCs}$ and $\mathrm{OHCs}$ contain mechanoelectrotransduction (MET) channels, which are gated by the movement of the stereocilia. The gating spring model was proposed to explain the gating of MET channels (Howard and Hudspeth, 1988). The tip links between stereocilia were suggested to work as springs ruled by Hook's law. When the stereocilia are deflected, this force compresses or extends the springs, which then push or pull the gate of MET channels, resulting in channel gating. Indeed, the displacement of stereocilia causes the MET channels to open; however, it also causes the gating springs to relax. This relaxation greatly reduces the stiffness of the stereocilia, and causes the opening of additional MET channels (Fettiplace, 2006). This active mechanical mechanism thereby amplifies mechanoelectrical transduction when the stimulus amplitude is weak. The electromotility of OHCs serves as a further means of mechanical amplification. OHCs contain a particular motor protein, prestin that is arrayed in high density in the lateral plasmalemma (Zheng et al., 2000). Prestin adjusts the length of OHCs in response to the fluctuation of membrane potential that result from mechanotransduction (Cheatham, 2004). Finally, the synergistic action of active bundle movements and electromotility in a specific cochlear partition greatly amplifies the vibration of the basilar membrane for soft sounds with high frequency selectivity.

The dynamic range for sound-intensity encoding spans over six orders of magnitude. However, the velocity of the vibration of the basilar membrane exhibits a nonlinear 
relationship with sound amplitude (Robles and Ruggero, 2001) and thereby compresses the range of inputs to MET. The velocity increases monotonically for weak sounds until a certain level, whereafter, the increase slows down (Ruggero et al., 1997). The reduction of moving velocity compresses the intensity of the sound by means of reducing the displacement of the basilar membrane to three orders of magnitude. This nonlinear compression is thought to enable the auditory system to grade the output of the cochlea (spike rate) for changes in input despite the limited dynamic range of sensory and neural mechanisms. The impeding force is considered to arise from the tectorial membrane, which is located above the $\mathrm{OHCs}$ and IHCs and behaves as the roof of the organ of Corti. Knocking out the key molecules composing the tectorial membrane, i.e., $\alpha$ - and $\beta$-tectorin, deteriorates the nonlinearity of the basilar membrane, in which the velocity of movement rises monotonically and linearly with the sound pressure level (Legan et al., 2000). Furthermore, removal of the tectorial membrane also attenuates amplification by OHCs (Legan et al., 2000). These observations demonstrate the mechanical function of the tectorial membrane in nonlinear compression.

After this complex processing of the mechanical signal the frequency is selected, loud sounds are compressed whereas weak sounds are amplified and finally, the IHCs are stimulated and form a receptor potential. IHCs innervate 10-30 spiral ganglion neurons (SGNs) (Matthews and Fuchs, 2010), which compose the auditory nerve. These SGNs are the first afferent neurons of the auditory system, and convey the signals via action potentials. Unlike SGNs, mature IHCs do not generate an action potential (AP). Instead, the fluctuating receptor potential of IHCs manifests the voltage changes caused by mechanotransduction. The graded receptor potential governs the $\mathrm{Ca}^{2+}$ dependent release of neurotransmitter from hair cells that in turn drives spiking of the SGNs. In other words, the function of afferent synapse between IHC and SGN is similar to an analog-digital converter, in which an analog sensory signal is encoded as a digital code. Interestingly, the synaptic ribbon, a special 
presynaptic structure dominates the hair cell afferent synapse, i.e., hence given the name "ribbon synapse."

\section{Ribbon synapses}

Ribbon synapses are structurally distinct and exist in cells that process environmental signals into the nervous system. These cells, including retinal photoreceptors and bipolar cells, electroreceptor cells, and cochlear hair cells, share a very important functional feature: release is controlled by a graded potential instead of an action potential (Juusola et al., 1996). These sensory cells must detect instantaneous changes in the environment and, most importantly, convey the signal faithfully to their downstream neurons. Moreover, compared with active zones of conventional synapses, ribbon-type active zones, both in retina and cochlea, possess more synaptic vesicles than conventional synapses (Sterling and Matthews, 2005). Likewise, vesicle recycling is necessarily efficient and indefatigable to support the incessant stimulation of these receptors by the environment (Moser and Beutner, 2000; Griesinger et al., 2005). Nevertheless, the detailed mechanism by which ribbon synapses organize this process is not clear.

Ribbon synapses have multiple shapes in different organs. In mouse IHCs, the synaptic ribbon is identified as a spherical, ellipsoidal, or bar-shaped electron-dense body under electron microscopy (Moser et al., 2006a; Nouvian et al., 2006). The size of these electron-dense bodies varies within and among the cell types. In addition, the size of the synaptic vesicle pool was reported to be also varied in different synapses (Moser et al., 2006a; Graydon et al., 2011) where larger ribbons tend to hold a larger pool of vesicles. There is great morphological heterogeneity in ribbon synapses, even within a single hair cell. The synaptic vesicles are either tethered to plasma membrane or to the synaptic ribbons via a 20-nm filament-like structure (Lenzi et al., 1999). Different from conventional synapses, 
the presence of synaptic ribbons sorts the docked vesicles into three populations. In addition to the vesicles docked directly onto or out of the active zone (AZ), one exceptional population of vesicles is sandwiched between the plasma membrane and the synaptic ribbons, and these are considered to be docked onto the plasma membrane (Lenzi et al., 2002; Sterling and Matthews, 2005). In frog saccular hair cells, the docked vesicles tethered to the synaptic ribbon are depleted before the other docked vesicles (Lenzi et al., 2002). This observation suggests that synaptic ribbons might play an important role in facilitating the release of synaptic vesicles through the interaction between the ribbons and the vesicles; however, how the ribbons promote vesicle recycling is still not clear.

RIBEYE, an alternative splice variant of the transcriptional repressor of dehydrogenase, C terminal binding protein 2 (CtBP2), is the main molecular component of the ribbon and is only expressed in ribbon synapses (Schmitz et al., 2000; Khimich et al., 2005). The homologous polymerization of RIBEYE constructs the body of the ribbon (Magupalli et al., 2008). RIBEYE has two different domains, i.e., an N-terminal A domain and a C-terminal B domain. The A domain is thought to have a structural role, whereas the $B$ domain is probably exposed in the cytosol, and is therefore suggested to have a metabolic function (Schwarz et al., 2011). With respect to vesicle tethering, Rab3-interacting molecule (RIM) was also identified on synaptic ribbons with its counterpart molecule, the synaptic vesicle protein Rab3 (Sterling and Matthews, 2005; Uthaiah and Hudspeth, 2010a). The interaction of RIM and Rab3 is supposed to facilitate the docking of vesicles to the ribbon (Sterling and Matthews, 2005). Two isoforms of RIM, RIM1 and RIM2, were identified in the chick cochlea; however, in mouse IHCs, it was recently suggested that RIM1 and RIM2 are only expressed before hearing onset (Gebhart et al., 2010). The movement of tethered and docked synaptic vesicles requires some motor proteins to drive their motion on the ribbons. KIF3a, a kinesin motor, is also reportedly a component of both retinal and cochlear ribbons (Muresan et al., 
1999). However, interrupting the polymerization of microtubules using low temperatures did not change ribbon morphology (Usukura and Yamada, 1987). Recently, myosin VI (myo6), an actin-based protein expressed on the synaptic ribbons and active zones of IHCs, was found to affect the maturation of synaptic ribbons as well as $\mathrm{Ca}^{2+}$ signaling (Roux et al., 2009). Therefore, myo6 could be a candidate motor protein, although there is still no evidence to indicate that it can function as a molecular motor.

Four scaffold proteins, Bassoon, Piccolo, CAST and ELKS have also been identified in synaptic ribbons (Dick et al., 2001; Khimich et al., 2005; tom Dieck et al., 2005; Uthaiah and Hudspeth, 2010a). Disruption of Bassoon impairs the auditory function and exocytosis of IHCs; the number of anchored ribbons is reduced in the mutant mouse, and floating ribbons were also observed in the cytosol, indicating the essential anchoring function of Bassoon (Khimich et al., 2005). Moreover, the fast component of exocytosis was also reduced in mutant hair cells in terms of membrane capacitance measurement. Whether the reduction of the exocytosis is directly cause by the disruption of Bassoon or caused by the loss of ribbon still needs to be clarified to understand the regulation of the ribbon on the exocytosis. By virtue of superresolution stimulating emission depletion microscopy, we can observe the misalignment of $\mathrm{Ca}^{2+}$ channel clusters, even in the ribbon-anchored synapses of Bassoon mutant mice. The results from this technique are consistent with observations made using freeze-fracture of frog saccular cells. In both cases, the line-like alignment of $\mathrm{Ca}^{2+}$ channels was observed (Roberts et al., 1990).

\section{Encoding of sound intensity in SGNs}

Sound is encoded as the rate and timing of firing of SGNs in response to hair cell transmitter release. Synaptic transmission at the hair cell ribbon synapse is very special. Changing the release probability modulates the rate of release and the EPSC amplitude at conventional 
synapses while the EPSC amplitude in SGNs remains nearly unchanged (Glowatzki and Fuchs, 2002; Goutman and Glowatzki, 2007; Grant et al., 2010) and its distribution matches that for spontaneous release. EPSCs vary dramatically in size which has been interpreted to reflect different extents of synchronized multivesicular release (Glowatzki and Fuchs, 2002; Grant et al., 2010). The mean of the EPSC is comparably much larger than that of the conventional synapse such as the auditory synapse in the brainstem e.g. calyx of Held (Meyer et al., 2001). The mechanism underlying the release process of the hair cell synapse is still not yet resolved.

In the postsynaptic end, the SGNs also have several specific features to transmit the information from IHCs. SGNs have a low threshold for generate an action potential and show a phasic response (Rutherford et al., 2012). Comparing with conventional central neurons, some SGNs were shown to exhibit higher spontaneous firing rate, which could even reach $100 \mathrm{~Hz}$ (Sachs and Abbas, 1974; Winter et al., 1990). Surprisingly, more than $80 \%$ of the spontaneous excitatory postsynaptic potential (EPSP) can generate an action potential (Siegel, 1992; Rutherford et al., 2012), which generally does not happen in conventional neurons. Voltage-clamp recording from the postsynaptic boutons and the immunostaining about spike generators reveals several important inherent features of SGNs. The first heminode of SGNs resides just beneath the IHCs (Hossain et al., 2005), suggesting that the action potential is triggered in close proximity of the postsynaptic bouton. In addition, the small size of the bouton lowers the membrane time constant, which can shortens the duration of membrane charging to the threshold of spiking (Rutherford et al., 2012). These characteristics ensure that SGNs can respond to the presynaptic stimulus with high accuracy. The other important feature of SGNs is their phasic excitability. SGNs only spike few times, mainly once in respond to long-duration stimulation, such as step-depolarization. This allows SGNs to filter out some other EPSP which is not elicited by the well-timed 
presynaptic releasing of neurotransmitters, thereby enhancing the precision of signal transferring in SGNs (Rutherford et al., 2012).

The spike rate of SGNs increases up to hundreds of $\mathrm{Hz}$ in response to sound stimuli and is essentially limited by the neuron's refractoriness. However, the mammalian auditory system can signal sound pressures that differ by 6 orders of magnitude. Even despite the nonlinear compression of basilar membranes, SGN still need to be able to respond to sound pressures that vary over thousand fold. In mammalian cochlea, SGNs show different acoustic thresholds. In vivo recordings from SGNs revealed heterogeneous response patterns (Sachs and Abbas, 1974). Therefore, SGNs work as different channels to the brain to enable the response of wide ranges of sound pressure. Different SGNs respond to sound intensity differently. Three response patterns for sound levels of the SGNs were identified: "saturating," "sloping-saturation," and "straight" modes (Figure I.1) (Winter et al., 1990). The SGNs for the saturating mode have the lowest acoustic threshold $(\sim 10-30 \mathrm{~dB})$ to fire action potentials but their firing rate does not increase after a certain level. The fibers for sloping-saturation and straight mode have higher thresholds; however, their firing rate does not saturate, even for very loud sounds. The firing rate increases monotonically in the straight mode, whereas it becomes slower in the sloping-saturation mode when the sound is louder than a certain level. Different types of auditory nerve fibers work together to cover different ranges of sound with different sensitivities; thereby broadening the total dynamic range of amplitude encoding in the auditory system.

Abbas and Sachs first tried to explain these patterns through the acoustic threshold of each individual fiber from mathematical modeling. They concluded that the pattern changes as a function of the threshold; in other words, the threshold determines which type of response 

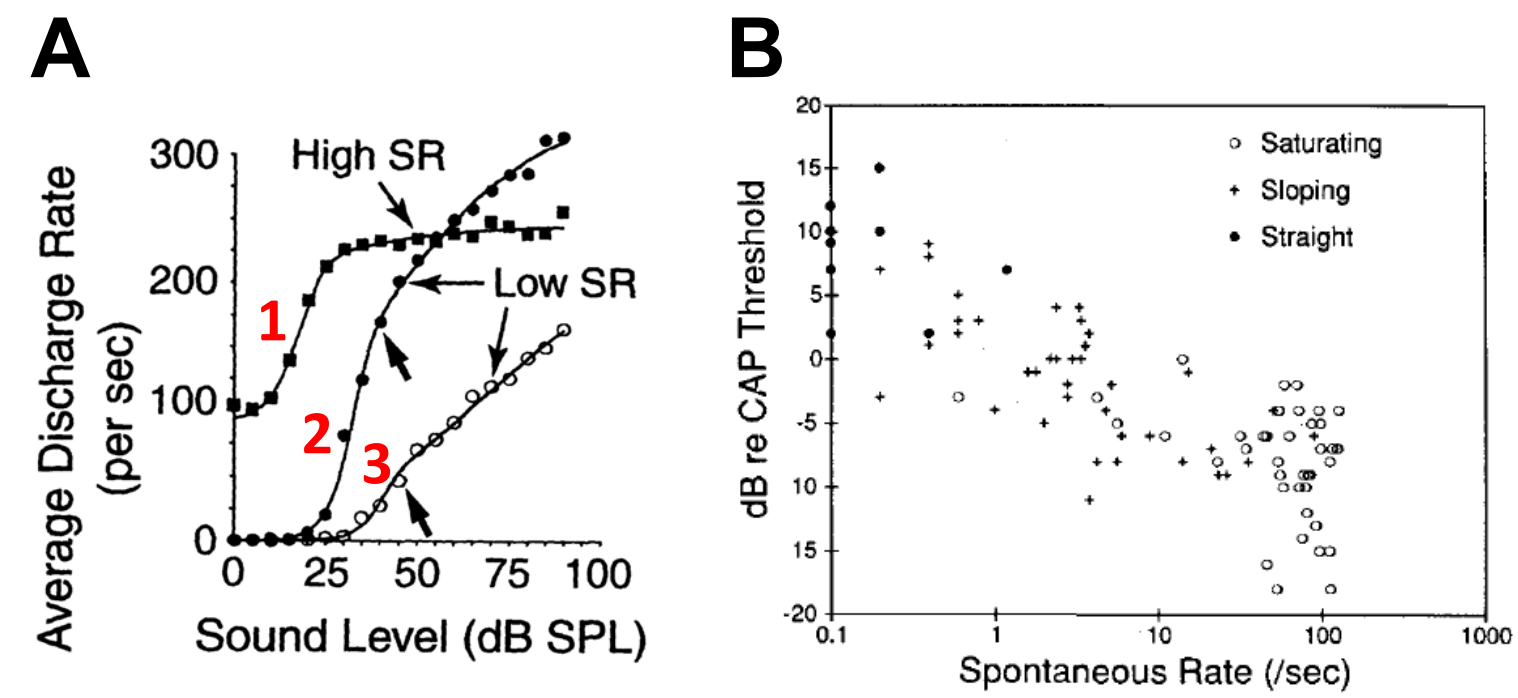

\section{Figure $\mathbf{l . 1}$}

The relationship between rate-level functions, spontaneous rates and auditory thresholds

(A) Different rate-level functions measured from different SGNs. Line 1,2 and 3 display different modes of rate-level functions: "saturating", "slope-saturation" and "straight" respectively. (from Geisler, 1998) (B) The SGNs with high spontaneous rates tend to have lower auditory thresholds and vice versa. Notice that the different symbols represent for different rate-level functions. This figure also indicates the "saturating" SGNs tend to have low auditory threshold and high SR and the "straight" SGNs tend to have higher threshold with low SR (figure from Winter et al. 1990).

patterns the auditory nerve generates (Sachs and Abbas, 1974). This hypothesis points out that the auditory threshold of a SGN is important in the determination of the dynamic range it can respond to; namely, the sensitivity of the SGNs. Interestingly, the auditory nerve fibers of nearby CF regions have quite diverse thresholds, from 10 to $100 \mathrm{~dB}$. These SGNs innervate neighboring IHCs, and probably some of them even innervate the same $\mathrm{IHC}$.

What determines the acoustic threshold of SGNs? Current-clamped recording from the postsynaptic bouton of SGNs reported neurons with lower spontaneous EPSP rate, however with high possibility to cause to the action potential successfully (Rutherford et al., 2012). The electric properties of SGNs, which is also mentioned above, are developed to be efficient to respond to the stimulus (Grant et al., 2010). The other possibility might arise from the presynaptic regulation. Voltage-gated $\mathrm{Ca}^{2+}$ channels expressed in IHCs respond to the 
graded potential and thereby control the neurotransmitter release by means of $\mathrm{Ca}^{2+}$ dependent exocytosis. $\mathrm{Ca}^{2+}$ imaging studies demonstrated both the heterogeneous intensity of synaptic $\mathrm{Ca}^{2+}$ influx and their voltage-dependent activation (Frank et al., 2009). Such heterogeneity might contribute to diversities of presynaptic regulation about acoustic threshold.

\section{$\mathrm{Ca}^{2+}$ signaling within hair cells}

$\mathrm{Ca}^{2+}$ plays many important roles in cells (Berridge et al., 2003). Neurons rely on the influx of $\mathrm{Ca}^{2+}$ to trigger the release of neurotransmitter that results in information transfer in the nervous system. The hair cell afferent synapse, which is the first synapse to transfer sound information, is equipped with a sophisticated mechanism to regulate the $\mathrm{Ca}^{2+}$-triggered release of neurotransmitter. Unlike conventional synapses, the ribbon synapses in hair cells must process a graded potential and transduce it faithfully into neurotransmission by means of the precise regulation of voltage-gated $\mathrm{Ca}^{2+}$ channels (Moser et al., 2006b). The major molecules to form $\mathrm{Ca}^{2+}$ conductance in IHCs is the low voltage-activated L-type $\mathrm{Ca}^{2+}$ channel $\mathrm{Ca}_{v} 1.3$ (Platzer et al., 2000). Hair cell $\mathrm{Ca}_{v} 1.3$ exhibits some particular characteristics, i.e., it has weak $\mathrm{Ca}^{2+}$-dependent inactivation (CDI) (Yang et al., 2006; Cui et al., 2007), a more negative activation threshold (Koschak, 2001), and fast activation and deactivation (Zampini et al., 2010). Some of these features are not observed when studying $\mathrm{Ca}_{v} 1.3$ channels in heterologous expression systems and, hence, the $\mathrm{Ca}_{v} 1.3$ channels must be subject to specific regulation in hair cells.

Acquiring the synaptic $\mathrm{Ca}^{2+}$ influx by means of the confocal $\mathrm{Ca}^{2+}$ imaging technique revealed submicrometer-size, spot-like fluorescence hotspots, which were colocalized with the fluorescently stained presynaptic ribbons. The intensity, spatial spread and voltage-dependence of the $\mathrm{Ca}^{2+}$ hotspots have been observed to exhibit great heterogeneity 
(Frank et al., 2009). The heterogeneity is reminiscent of the diverse rate-level functions of the different SGNs, which can originate from different ribbon synapses as well as their $\mathrm{Ca}^{2+}$ channels. Hence, understanding the mechanisms of the $\mathrm{Ca}^{2+}$ heterogeneity might be the key to explain the different patterns of the rate-level functions. Several presynaptic mechanisms can cause the heterogeneity. The $\mathrm{Ca}^{2+}$-induced $\mathrm{Ca}^{2+}$ release appeared not to contribute much to the heterogeneity of $\mathrm{Ca}^{2+}$ hotspots (Frank et al., 2009). The most promising mechanisms of $\mathrm{Ca}^{2+}$ hotspot heterogeneity include varying $\mathrm{Ca}^{2+}$ channel numbers, different modulatory impact of auxiliary subunits of $\mathrm{Ca}^{2+}$ channels, different splice variants of Cav1.3 $\alpha_{1 \mathrm{D}}$ or the varying regulatory function of other proteins or lipid.

A number of proteins have been shown to interact with $\mathrm{Ca}^{2+}$ channels and to manipulate their function. Increasing the $\mathrm{Ca}^{2+}$ concentration can enhance the interaction of $\mathrm{Ca}^{2+}$ channels with numerous $\mathrm{Ca}^{2+}$ binding proteins and result in $\mathrm{CDI}$ or facilitation (CDF) (Calin-Jageman and Lee, 2008). The mechanisms by which calmodulin generates CDI and CDF have been well investigated. Calmodulin attaches to the IQ domain of the C-terminal of L-type calcium channels after binding $\mathrm{Ca}^{2+}$ to its $\mathrm{C}$ lobe, resulting in CDI (Peterson et al., 1999). Deletion of the IQ domain of $\mathrm{Ca}_{v} 1.3$ reportedly hampers $C D I$ (Shen et al., 2006). Ca ${ }^{2+}$ and calmodulin-dependent inactivation has been reported in cochlea hair cells (Yang et al., 2006; Cui et al., 2007). In addition to calmodulin, members of the calmodulin-like $\mathrm{Ca}^{2+}$-binding protein (CaBP) family also affect $\mathrm{CDI}$; however, in the opposite way. The overexpression of CaBP1 and CaBP4 in human embryonic kidney (HEK) cells eliminates CDI (Yang et al., 2006; Cui et al., 2007). It is supposed that CaBPs might perturb the normal binding of calmodulin to $\mathrm{Ca}_{\sqrt{ }} 1.3$, probably by competing with binding to the IQ domain and causing the attenuation of CDI (Yang et al., 2006). Such a phenomenon also occurs in IHCs. IHCs display considerably weak CDI in comparison with neurons or cell lines. In mouse IHCs, multiple CaBPs have been detected, including CaBP1, CaBP2, CaBP4, and CaBP5. 
However, knocking out CaBP4 did not impair auditory function; instead, CaBP1 has been shown to colocalize with synaptic ribbons and might be a potential interacting partner with Cav1.3 in IHCs (Cui et al., 2007). Besides CaBP1 and CaBP4, CaBP2 was proven to be a deafness gene (Schrauwen et al., 2012); therefore, it might also contribute to the inhibition of $\mathrm{CDI}$ in IHCs. Other molecules such as $\mathrm{Ca}^{2+} /$ calmodulin-dependent protein kinase II (CaMKII) (Jenkins et al., 2010) and A-kinase anchoring protein (AKAP79/150) (Oliveria et al., 2007) reportedly regulate the activity of L-type $\mathrm{Ca}^{2+}$ channels through binding to the channels, but their effect on cochlear hair cells has not yet been examined.

The behavior of the Cav1.3 $\mathrm{Ca}^{2+}$ channels is also determined by the choice of the specific splice variant of their pore-forming $\alpha$ subunit and the precise composition of the channel complex (auxiliary subunits). An intrinsic C-terminal modulator (CTM) of the $\mathrm{Ca}_{\mathrm{v}} \alpha 1.3$ subunit interacts intermolecularly and intramolecularly to induce the modulation of gating and the inactivation of $\mathrm{Ca}_{v} 1.3$ (Koschak, 2010; Tan et al., 2011a). The C-terminus of $\mathrm{Ca}_{\mathrm{v}} \alpha 1.3$ constitutes approximately $25 \%$ of the total channel protein, and is encoded by 11 exons from exons 39 to 49 . Interestingly, numerous alternative splice variants of the Cav1.3 1 subunit have been identified in the nervous system. Alternative splicing of these exons can vary the intermolecular or intramolecular interactions of the $\alpha$ subunit (Lipscombe et al., 2002). For example, a frame-shift occurring between exons 41 and 42 causes the termination of transcription and generates a short isoform, $\mathrm{Ca}_{\mathrm{v}} 1.3_{42 \mathrm{~A}}$. In HEK cells, Ca $\mathrm{V}_{\mathrm{v}} 1.3_{42 \mathrm{~A}}$ expression shows a negative shift of activation voltage and enhances CDI (Singh et al., 2008). Alternative splicing of exon $43\left(\mathrm{Ca}_{\mathrm{v}} 1.3_{43 \mathrm{~s}}\right)$ caused a similar effect to $\mathrm{Ca}_{\mathrm{v}} 1.3_{42 \mathrm{~A}}$ (Tan et al., 2011a); however, the phenotype of the full-length isoform could be restored with both splice variants by the additional expression of the most distal 116 amino acids $\left(\Delta \mathrm{C}_{116}\right)$ (Singh et al., 2008; Tan et al., 2011a). Moreover, experiments using FRET (fluorescence resonance emission transfer) further proved the interaction of $\Delta \mathrm{C}_{116}$ to the PCRD (proximal C-terminal 
regulator domain), in which several amino acids in the most distal C-terminus of $\mathrm{Ca}_{v} 1.3_{42 \mathrm{~A}}$ and $\mathrm{Ca}_{\mathrm{v}} 1.3_{43 \mathrm{~S}}$ are included (Singh et al., 2008). This result implicated intramolecular interactions in $\mathrm{Ca}_{\mathrm{v}} 1.3$. Besides, alternative splice variants of the IQ domain at the C-terminus of $\alpha 1 \mathrm{D}$ have been identified in the cochlea, especially in OHCs (Shen et al., 2006). The endogenous expression of this alternative splice variant is expected to impact on CDI in OHCs. In addition to the numerous splice variants in the C-terminus, other alternative splice variants of domain linkers have been found in chick cochlear hair cells (Kollmar et al., 1997). These variants were also shown to affect the activation kinetics of the channels (Koschak, 2001; Xu and Lipscombe, 2001); however, the function of these three splicing variants has not been examined in hair cells.

Beside the $\alpha$ subunit, the $\beta$ subunit reportedly governs the trafficking and function of L-type $\mathrm{Ca}^{2+}$ channels. Knocking out the $\mathrm{Ca}_{\mathrm{v}} \beta 2$ subunit in IHCs greatly attenuated the $\mathrm{Ca}^{2+}$ current, and a reduced intensity of $\mathrm{Ca}_{\mathrm{v}} 1.3$ staining was observed (Neef et al., 2009). The reduction of $\mathrm{Ca}^{2+}$ influx might be attributed to the degradation of $\mathrm{Ca}^{2+}$ channels because the $\beta 2$ subunit could prevent the ubiquitination of L-type $\mathrm{Ca}^{2+}$ channels (Altier et al., 2010). Other auxiliary subunits, such as the $\alpha 2 \delta$ subunit, also regulate the abundance of $\mathrm{Ca}^{2+}$ channels (Hoppa et al., 2012).

$\mathrm{Ca}_{v} 1.3$ colocalizes at the submicrometer range with synaptic ribbons in mature hair cells indicating their clustering at the presynaptic active zone (Brandt et al., 2005; Frank et al., 2009). This close association greatly reduces the distance that incoming $\mathrm{Ca}^{2+}$ has to travel to the $\mathrm{Ca}^{2+}$ sensors on the synaptic vesicles (Naraghi and Neher, 1997), and thus allows for high sensitivity to changes in membrane potential. Distinct vesicle pools can be separated by $\mathrm{Ca}^{2+}$ chelators with different binding kinetics. Confining the spreading ranges of $\mathrm{Ca}^{2+}$ influx by fast kinetic $\mathrm{Ca}^{2+}$ chelator, i.e. BAPTA, can cause stronger attenuation of membrane 
capacitance increase than that of EGTA, which suggests a fast pool exists in several tens of nanometer proximity to $\mathrm{Ca}^{2+}$ channels (Moser and Beutner, 2000; Graydon et al., 2011). Similar phenomenon was observed in the pair recording of IHCs and the boutons of SGNs where application of BAPTA can cause more reduction of EPSC rate than that of EGTA (Goutman and Glowatzki, 2007). Furthermore, altering the opening numbers of $\mathrm{Ca}^{2+}$ channels by the application of dihydropyridine revealed a positive linear relationship between charges of $\mathrm{Ca}^{2+}$ influx and the exocytic increase of membrane capacitance. In contrast, altering the single channel conductance revealed a nonlinear relationship while the enhancement of exocytosis is saturated after certain level of influx $\mathrm{Ca}^{2+}$ (Brandt et al., 2005). This result further implicated that few opening $\mathrm{Ca}^{2+}$ channels can govern the release of near synaptic vesicles whereby support the regulation of $\mathrm{Ca}^{2+}$ nanodomains instead of microdomains. In physiological conditions, the abundant endogenous buffers calbindin-28k and parvalbumin- $\alpha$ have roles in the control of $\mathrm{Ca}^{2+}$ diffusion from the open mouths of the channels which is analog to the effect of applying $0.1 \sim 0.5 \mathrm{mM}$ EGTA, and thereby separate the different releasing characteristics and pool recycling (Hackney et al., 2005).

\section{Aims of this work}

To understand the synaptic mechanisms of sound amplitude encoding, it is crucial to understand the origin of the heterogeneous firing properties of SGNs. As mentioned above, the diversity of the $\mathrm{Ca}_{v} 1.3$ can cause different functional properties of $\mathrm{Ca}^{2+}$ signaling. Hence, this work aims to characterize the heterogeneity of synaptic $\mathrm{Ca}^{2+}$ signaling in IHCs. The intensity of $\mathrm{Ca}^{2+}$ influx among different synapses will be examined and compared. Besides

that, the voltage-dependent properties of $\mathrm{Ca}^{2+}$ channels activation are also an interesting hypothesis to explain this heterogeneity, and will be investigated.

As a relationship between site of insertion at the IHC and function of a given SGN was 
indicated previously, I will also analyze the spatial dependency of the properties of the synaptic $\mathrm{Ca}^{2+}$ microdomains and relate the results to in vivo recordings of SGNs. 


\section{Materials and Methods}

\section{Animals}

C57BL/6 mice (ages 14-18 days) were used for experiments.

\section{Preparations of the organ of Corti}

Mice were euthanized and the heads were transferred onto ices. After removing the skin, the skull was cut sagitally in half and transferred into a Petri dish containing ice-cold HEPES-buffered Hanks' balanced salt solution (see "Solutions" in Material \& Method). The brain tissue was removed and the cochlea together with the rest of the bony labyrinth was gently pulled out of the skull with a pair of No.3 forceps. After the bony walls of the cochlea were carefully peeled off piece by piece from the apex, the first-half apical turn of the organ of Corti was explanted from the modiolus and transferred to the next step by a fire-polished Pasteur's pipette.

After isolating the organ of Corti as described above, the whole-mount preparation was transferred to a recording chamber and fixed with a grid of nylon threads, with the basilar membrane down and the tectorial membrane up. During recordings, modified Ringer's solution (see "Solutions") was constantly perfused at a rate of at least $0.5 \mathrm{ml} / \mathrm{min}$. The tectorial membrane was first removed from the spiral limbus with a large-opening $(\varnothing \sim 20-40$ $\mu \mathrm{m})$ pipette and thereby revealed the IHCs and other tissues. To access the IHCs from the side of modiolus, the other cleaning pipettes with medium opening size $(\varnothing \sim 3-5 \mu \mathrm{m})$ were used to clean the supporting cells and compress the spiral limbus. The supporting cells, i.e. inner border cells, inner sulcus cells and phalangeal cells, were deteriorated by removing their nucleus. After a minute, a gentle negative pressure was applied to drain their debris. Then, to obtain a clean, patchable membrane at the IHC, another cleaning pipette with small 
opening $(\varnothing \sim 1-2 \mu \mathrm{m})$ was used to clean the surface of IHCs by either sucking or blowing gently, and finally revealed the patchable IHCs.

\section{Whole-cell patch-clamp recordings}

Patch pipettes were pulled from GB150-8P borosilicate glass capillaries (Science Products, Hofheim, Germany) with a Sutter P-2000 laser pipette puller (Sutter Instrument Company, Novato, USA). The opening size of the pull pipette was around $1 \mu \mathrm{m}$. The pulled pipettes were then polished by the custom-made microforge to smooth the tip of the pipettes thereby to improve the quality of "giga-seal".

The patch-clamp setup was shielded by custom-made Faraday cage and assembled on hydraulic air table for vibration isolation (TMC, Peabody, USA). Experiments were performed with a Zeiss Axio microscope (Carl Zeiss microscopy GmbH, Göttingen, Germany) equipped with Zeiss $63 x$ water immersion objective "W Plan-Apochromat 63x/1,0 M27" (1.0 NA). The objective was mounted on a piezoelectric focusing motor (MIPOS 100 PL, Piezosystem Jena, Jena, Germany), and was controlled by the piezo controller (NV 40/1 CLE, Piezosystem Jena, Jena, Germany). Patch pipettes were approached towards the preparation with a motorized micromanipulator (MP-285, Sutter Instrument, Novato, USA). Patch-clamp recordings were performed with EPC-10 USB amplifiers (HEKA, Lambrecht/Pfalz, Germany) operated by PatchMaster software (HEKA).

Patch pipettes approached the IHCs with a gentle positive pressure around $5 \mathrm{~cm} \mathrm{H}_{2} \mathrm{O}$. Right after the appearance of a small dimple on IHCs, the positive pressure was released thereby resulting in an increasing seal resistance $\left(R_{\text {seal }}\right)$. After the sealing resistance rose above 5 $\mathrm{G} \Omega$, the clamping voltage was tuned to $-70 \mathrm{mV}$ (before the correction of liquid junction potential), and the fast capacitance $\left(\mathrm{C}_{\text {fast }}\right)$ caused from patch pipette was compensated by 
the automatic routines in PatchMaster and EPC-10 USB. Afterwards, a series of gentle suction pulses applied by mouth were used to rupture the patch membranes. After successful rupture, the series resistance $\left(R_{\text {series }}\right)$ from the junction between the pipette and the cell was cancelled together with the cell membrane capacitance $\left(\mathrm{C}_{\text {slow }}\right)$ simultaneously by the automatic routines.

The quality of patched cells was judged by the $R_{\text {seal }}$ and $R_{\text {series. }}$. The $R_{\text {seal }}$ was mostly above 1 $\mathrm{G} \Omega$, and $\mathrm{R}_{\text {series }}$ was always below $15 \mathrm{M} \Omega$ before the compensation of series resistance. Liquid junction potential of patch pipettes was calculated by Patcher's Power Tools macros in Igor Pro (Wavematrics, Lake Oswego, USA), and the value was around $-17 \mathrm{mV}$. The voltage shift caused by the ion current flowing through the $R_{\text {series }}$ was corrected together with the liquid junction potential to obtain the real clamped voltage of cell membranes.

\section{Spinning disk confocal microscopy}

Fluorescently labeled IHCs were imaged using spinning disk confocal scanning. Detailed function and specification is described in the review (Gräf et al., 2005). The spinning disk scanner (CSU22, Yokogawa Electric Cooperation, Tokyo, Japan) was mounted on the Zeiss Axio microscope, and fixed mechanically with custom-made metal bar (fig III.2) to reduce the vibration from the disk spinning.

Fluorescence images were acquired by a back-illuminated CCD camera with $80 \times 80$ pixels (NeuroCCD, Redshirt Imaging, Decatur, GA, USA ) of which acquired images were magnified by an 2.5x after-magnification tube (VM Lens C-2.5x, Nikon, Tokyo, Japan). The tube and camera were mounted on top of the CSU22 accordingly. The pixel-resolution of the acquired image was $164 \mathrm{~nm}$, and the lateral and axial resolution of the optics were determined by $100 \mathrm{~nm}$ fluorescence beads (Fluospheres, Invitrogen, Grand Island, NY, 
USA), and were $\sim 300 \mathrm{~nm}$ and $1.6 \mu \mathrm{m}$ respectively. Image data were acquired and processing by the Neuroplex software (Redshirt Imaging).

Images were acquired in $10 \mathrm{~ms} / \mathrm{frame}, 5 \mathrm{~ms} /$ frame and $50 \mathrm{~ms} /$ frame depending on the experiment requirements. Synchronizing the disk spinning speed to the camera frame rate is necessary to avoid the uneven illumination (fig III.1). In these conditions, the spinning speed was set to be $2000 \mathrm{rpm}$.

The green fluorescence from the $\mathrm{Ca}^{2+}$ indicator Fluo-8FF was excited by the $491 \mathrm{~nm}$ diode-pump solid-state laser (Calypso, Cobolt AB, Solna, Sweden), and the red fluorescence from TAMRA was excited by the $561 \mathrm{~nm}$ diode-pump solid-state laser (Jive, Cobolt $A B$ ). Gating of the laser was operated by the electro-programmable shutter system (LS6 and VCM-D1, Uniblitz Co., Rochester, NY, USA). The laser was guided into the single-mode optic fiber (Oz optics, Ottawa, Ontario, Canada) through the coupler (KineFLEX, Qioptiq, Luxembourg).The pathway of laser in the scanner is demonstrated as fig II.1. The

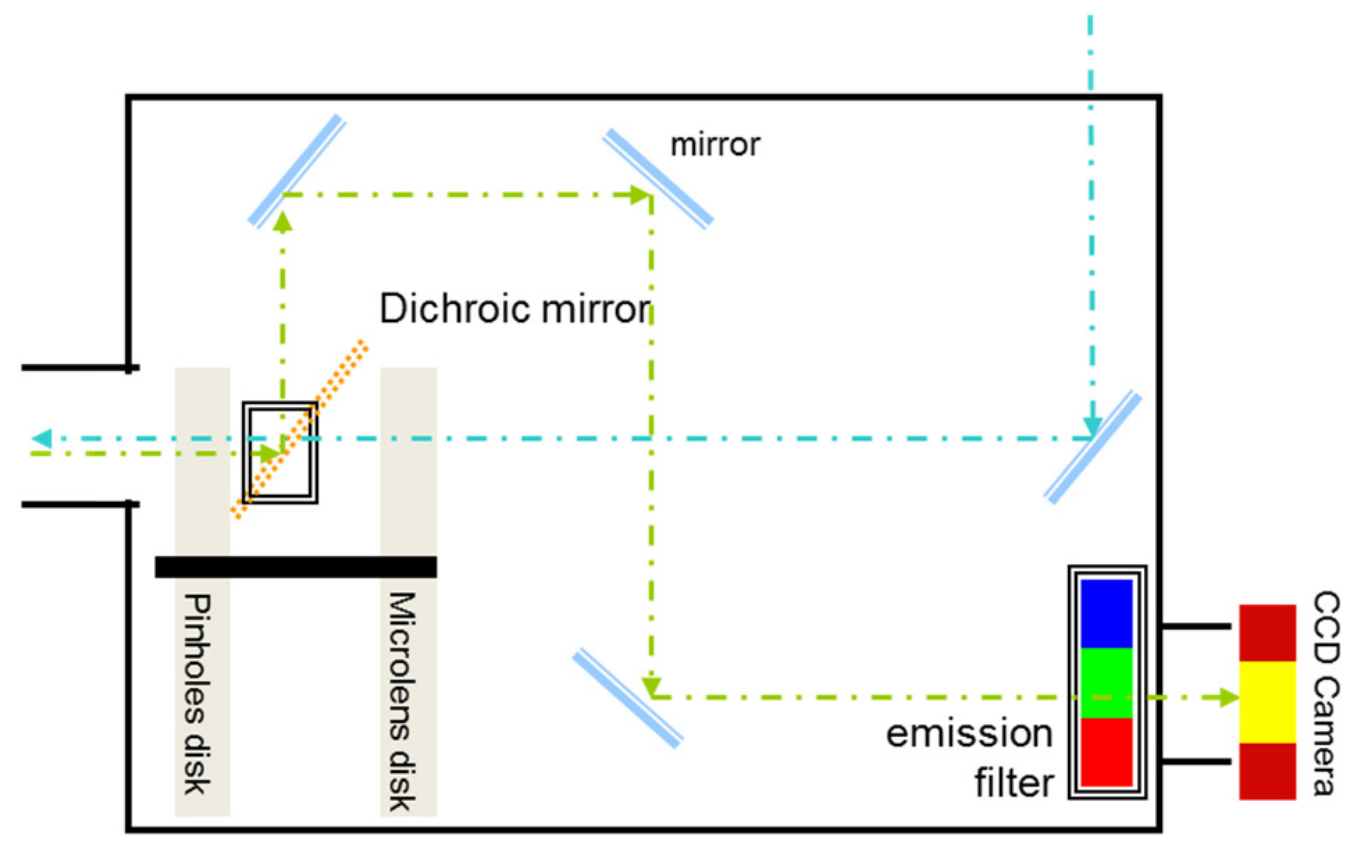

Figure II.1 Pathway of light in the spinning disk scanner

The blue dash line is the excitation beam, and green dash line is the emission light. Other units are represented as the labels. 
$491 \mathrm{~nm}$ excitation was transmitted by a short pass dichroic mirror (Di01-T488, Semrock Inc, Rochester, NY, USA) and the resulting green emission was reflected by this dichroic mirror to the CCD camera. An additional long-pass emission filter (BLP01-488R-25, Semrock Inc.) was used to clean up the remaining 491 excitations light in front of the camera. The 561 nm excitation was transmitted by a multi band-pass dichroic mirror (Di01-T405/488/568/647, Semrock Inc) and resulting red fluorescence was reflected by it. The other additional band-pass emission filter (HQ608/60m BP, Chroma, Bellows Falls, VT, USA) was used to pass the light of the wavelength of $578-638 \mathrm{~nm}$ and block the $561 \mathrm{~nm}$ laser.

\section{$\mathrm{Ca}^{2+}$ imaging of $\mathrm{IHCs}$}

After the formation of whole-cell patch and break-in, the $800 \mu \mathrm{M} \mathrm{Ca}^{2+}$ indicator Fluo-8FF (AAT Bioquest, Sunnyvale, CA, USA) was subsequently dialyzed into the cell with the $\mathrm{Cs}^{+}$-based intracellular solution (for the recipe see "Solutions") for more than 4 minutes to reach a steady-state concentration. In addition to Fluo-8FF, $20 \mu \mathrm{M}$ TAMRA-conjugated C-terminal binding protein 2 (CtBP2) binding peptide was also contained in the intracellular solution and diffused into the IHCs to visualize the synaptic ribbons. This dimeric peptide was described by the Zenisek lab (Francis et al., 2011). The sequence of this peptide is as follows:

(TAMRA)-EQTVPVDLSARPR-(PEG6-PEG6)-EQTVPVDLSARPK-(TAMRA)-amide

After 5 minutes of perfusion, the position and fluorescence intensity of the labeled ribbons were first scanned from the basal end of IHCs to the top of nucleus, the step size of each movement was $0.5 \mu \mathrm{m}$. $\mathrm{Ca}^{2+}$ imaging was subsequently performed in the range determined by the first scanning of ribbons. To avoid the errors caused by bleaching of fluorescence in the later sections, imaging acquisitions were applied in jumping order among sections. 
Patched IHCs were first voltage clamped in $-87 \mathrm{mV}$. To evoke the fluorescent $\mathrm{Ca}^{2+}$ hotspots, $50 \mathrm{~ms}$ depolarizations of square pulse to $-7 \mathrm{mV}$ were applied in every scanned section during acquisitions. The interval between 2 sequential sections was $2 \mathrm{~s}$ to eliminate the effect of CDI. After the first scanning of $\mathrm{Ca}^{2+}$ imaging, ramp depolarizations from $-70 \mathrm{mV}$ to $+30 \mathrm{mV}$ in $100 \mathrm{~ms}$ were applied to investigate the activation of $\mathrm{Ca}^{2+}$ hotspots in every section, and the images were acquired with $5 \mathrm{~ms}$ per frame. This protocol was run at most 3 times depending on the stability of the $\mathrm{Ca}^{2+}$ current and the viability of the cells.

\section{$3 D$ reconstruction of $I H C s$}

After all the $\mathrm{Ca}^{2+}$ imaging experiments, IHCs were then scanned in the red channel to acquire the morphology. Scanning began in the section which was $1 \mu \mathrm{m}$ below the fluorescence-visible basal end to ensure full scanning of IHCs, and then images were acquired in the direction toward the apical end of IHCs. However, owing to the small size of CCD camera, scanning in fixed field of view was usually not sufficient to include an entire cell. The field of view was therefore shifted in-between to include all the portion of the given IHC. The acquired image stacks were then processed in Igor Pro software (Wavemetrics) to assemble into an entire 3D image of IHC.

To transfer the images from canonical Cartesian coordinates into self-defined cylindrical coordinates, 3 orthogonal reference axes and one reference point are required to be defined. In the previous study (Meyer et al., 2009), the information of the cochlear axial axis, "tonotopical" axis, was taken from confocal images of immunostained IHCs. However in the current patch-clamp and $\mathrm{Ca}^{2+}$ imaging experiments, this information could not be reliably obtained because of inevitable pipette-manipulation of cells and the small image frame. Instead of tonotopical axis, we adopt the inherent characteristics of cell shape, the plane of 
symmetry and its vector $\left(\mathrm{V}_{\text {sym }}\right)$, as a surrogate of tonotopical axis (fig III.8). $\mathrm{V}_{\text {sym }}$ was obtained from the cross product of 2 vectors. These 2 vectors were determined by the center of mass (C.M.) of the IHC cytosolic fluorescence in confocal sections. The first vector connected the C.M. of 2 sections along the Cartesian $Z$ axis. One of them was close to the basal end, and the other is close to the nucleus. The second vector also connected the C.M. of 2 sections along the $y$ axis. Cross products of these 2 vectors produce the $V_{\text {sym }}$, the quality of $V_{\text {sym }}$ was then judged by eye such as in the visualized cell of fig III.8a. to exclude obviously unsymmetrical cases

After obtaining the plane of symmetry, the second axis and the reference point were specified on this plane. I used the pillar cell contact of the IHC as second axis. Because this line lay on the plane of symmetry, it was orthogonal to $V_{\text {sym. }}$. Next step the cell was resampled the along the axis of this pillar line, $V_{z}$, by self-made macros in Igor Pro. Then I specified the center of mass of the largest cross-section of the $\mathrm{IHC}$ in the resampled images as the center point (reference point) of the cell (fig III.8b). The third axis $V_{m p}$ was the cross product of $\mathrm{V}_{\text {sym }}$ and $\mathrm{V}_{\mathrm{z}}$. By $\mathrm{V}_{\mathrm{z}}, \mathrm{V}_{\mathrm{sym}}, \mathrm{V}_{\mathrm{mp}}$, and the center, the Cartesian $(\mathrm{x}, \mathrm{y}, \mathrm{z})$ coordinate can be transferred to cylindrical $(r, \theta, H)$ coordinate. The $r$ represents as the distance between the spot and the center of mass (green dot with red ring in fig III.8c) of the section that contains the spot. " $\theta$ " is the angle from $V_{\text {sym }}$ to $r$, and $H$ is the distance from spot-containing section to the center point section. In the polar chart of results, $\theta$ from 0 to 180 degrees represents the modiolar face of the IHC, and 180 to 360 degrees the pillar face of the IHC. 90 to 270 degrees are apical 270 to 90 degrees are basal with respect to tonotopic axis.

\section{Data analysis}

The $\mathrm{Ca}^{2+}$ imaging and patch-clamp data were analyzed in Igor Pro with numerous custom macros. The 3D images of IHCs were visualized by Fiji software (open source software). 
6 images preceding the depolarization were averaged to be the reference images for the resting conditions. When using square pulse depolarizations, the 4 frames between $10 \mathrm{~ms}$ to $50 \mathrm{~ms}$ after the onset of depolarization were averaged as an image of the stimulation. Subtracting the resting from the stimulated image produced the $\Delta \mathrm{F}$ image, where the evoked $\mathrm{Ca}^{2+}$ indicator fluorescence change can be seen by eye. In $\Delta \mathrm{F}$ images, intensities of the 8 pixels surrounding the central pixel of the fluorescence punctum were averaged with that of the central pixel, the mean of this calculation was taken as the measured intensities $\left(\Delta \mathrm{F}_{\text {avg }}\right)$ of the fluorescent $\mathrm{Ca}^{2+}$ hotspots.

To obtain the fluorescence intensity of the stained ribbons, 9 images excluding the $1^{\text {st }}$ image from the same scanning section were averaged then subsequent estimation of the fluorescent-ribbon intensity was performed on this averaged image. The intensities of ribbon stains were the intensities of the central pixel of visible fluorescence puncta from this averaged image.

Fluorescence-voltage (FV) relationships were estimated also from ramp depolarization experiments. In those experiments, the region to estimate the intensity of each $\mathrm{Ca}^{2+}$ microdomain was similar to the method described in the previous paragraph. The intensity was measured in every frame and the resulting values were then related to their corresponding voltages to produce the FV curves.

The raw FV curves were afflicted with noise such as readout noise or shot noise from the CCD camera. Hence, the following equation was used to fit then to optimize the raw traces:

$$
F(V)=F_{0}+\frac{g_{\max }\left(V-V_{r}\right)}{1+e^{\left(V_{0.5}-V\right) / k}}
$$

The fitting result is demonstrated in figure II.2. All the fitting parameters were automatically generated by the Igor Pro software, which were only used for obtaining a good fitting result. The fits to the FV curves (FV-fits) were then used for further analysis. The goodness of fitting 
was judged by overlapping the raw trace to the fitting curve. Few curves were not fit properly, for example, the gray curve in figure II.2. The ill-fitted data were not used for further analysis.

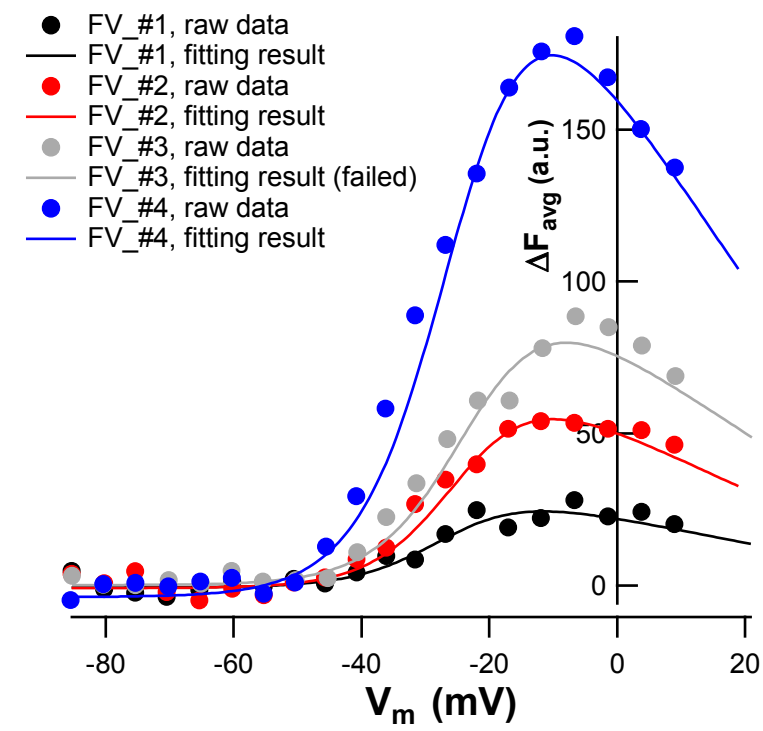

Figure II.2 Refining raw FV curves

The dots represent for the raw data points and the lines represent for the corresponding fitting result. 4 FV curves obtained from different $\mathrm{Ca}^{2+}$ microdomains are listed in this figure in different colors. Notice that the gray curve, FV_\#3 was fitted poorly and thereby was not used for subsequent analysis.

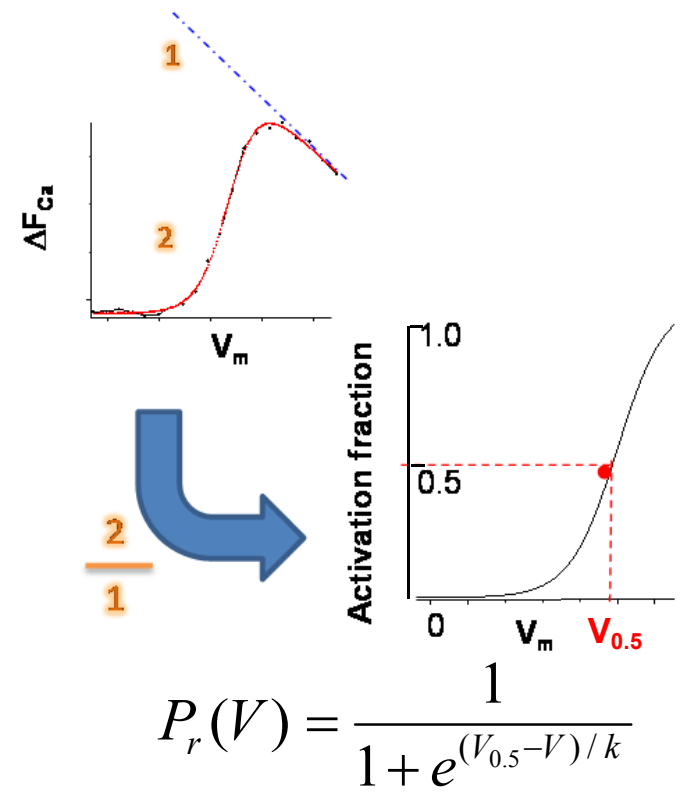

Figure II.3

Analysis routine of the voltage-dependent activation of fluorescent $\mathrm{Ca}^{2+}$ indicator hotspots.

The fractional activation curve was obtained by dividing the FV trace ("2" in the figure) by the full-activation line (" 1 " in the figure).The resulting fractional activation curve was then fitted by the Boltzmann function as the equation in this figure to obtain the fitting parameter $\mathrm{V}_{0.5}$ and $\mathrm{k}$.

The FV-fits were subsequently divided by a line, which approximated the decline of fluorescence at depolarized voltages which results from the declining driving force despite full activation (Line "1" in figure II.3). These lines were obtained by connecting the intensity of $+10 \mathrm{mV}$ (before the correction of LJ potential) from the FV-fits to the reversal potential of $\mathrm{Ca}^{2+}$ measured by the IV of the patch-clamp data. Then these lines were extended to all the voltage of the corresponding FV traces to estimate the assumed fluorescence intensity of 
every voltage in the full activation condition. The resulting curves were the fractional activation curves. The fractional activation curves were then fitted by the Boltzmann function to obtain the parameters (Figure II.3): voltages for half activation $\left(\mathrm{V}_{0.5}\right)$ and slope-factor $(\mathrm{k})$.

\section{Solutions}

Modified Ringer's solution with $5 \mathrm{mM} \mathrm{Ca^{2+ }}$

$\mathrm{NaCl} 102.2 \mathrm{mM}, \mathrm{KCl} 2.8 \mathrm{mM}, \mathrm{MgCl}_{2} 1.0 \mathrm{mM}, \mathrm{CaCl}_{2} 5.0 \mathrm{mM}, \mathrm{CsCl} 1.0 \mathrm{mM}$, TEA-Cl $35.0 \mathrm{mM}$, HEPES $10.0 \mathrm{mM}$, glucose $2 \mathrm{~g} / \ell$. $\mathrm{pH}$ was adjusted to $7.2-7.3$ by $1 \mathrm{M} \mathrm{NaOH}$ solution. The osmolarity was around $300 \mathrm{mmol} / \ell$.

\section{HEPES-buffered Hank's balanced salt solution}

$\mathrm{NaCl} 141.7 \mathrm{mM}, \mathrm{KCl} 5.36 \mathrm{mM}, \mathrm{MgCl}_{2} 1.0 \mathrm{mM}, \mathrm{CaCl}_{2} 0.1 \mathrm{mM}, \mathrm{MgSO}_{4} \cdot 6 \mathrm{H}_{2} \mathrm{O} 0.5 \mathrm{mM}$, HEPES $10.0 \mathrm{mM}$, glucose $1 \mathrm{~g} / \ell$, L-Glutamine $500 \mathrm{mg} / \ell$. $\mathrm{pH}$ was adjusted to $7.2-7.3$ by $1 \mathrm{M} \mathrm{NaOH}$ solution. The osmolarity was around $300 \mathrm{mmol} / \ell$.

\section{$\underline{C s^{+} \text {-based Intracellular solution }}$}

L-glutamate $123.0 \mathrm{mM}, \mathrm{MgCl}_{2} 1.0 \mathrm{mM}, \mathrm{CaCl}_{2} 1.0 \mathrm{mM}$, EGTA $10.0 \mathrm{mM}$, TEA-Cl $13.0 \mathrm{mM}$, HEPES $20.0 \mathrm{mM}$, Mg-ATP $2.0 \mathrm{mM}$, Na-GTP $0.3 \mathrm{mM}$. pH was adjusted to $7.2-7.3$ by $1 \mathrm{M} \mathrm{NaOH}$ solution. The osmolarity was around $310 \mathrm{mmol} / \ell$. Right before every experiment, the stocked solution of $10 \mathrm{mM}$ Fluo-8FF was diluted into the intracellular solution to be $0.8 \mathrm{mM}$. The stock solution of $10 \mathrm{mM}$ TAMRA-conjugated CtBP2 peptide was diluted as well, and the final concentration was $20 \mu \mathrm{M}$. After the dilution of Fluo-8FF and peptide, the osmolarity was reduced to be around $300 \mathrm{mmol} / \ell$. 


\section{$\underline{\text { Results }}$}

\section{Spinning disk confocal microscopy}

During our physiological experiments, IHCs were embedded in the organ of Corti. When observing the fluorescence of indicators, the depth of the tissue and cell would cause blur images because the fluorescence of the non-focal plane were also sampled. Therefore, confocal microscopy was employed to reduce the out-of-focus fluorescence and to better resolve individual synapses in an IHC. In my PhD project, I aimed examine the behavior of several evoked fluorescent $\mathrm{Ca}^{2+}$ indicator hotspots simultaneously in several ten to hundred milliseconds. To study synaptic functions, acquiring a frame in very short duration such as 10 ms or shorter is necessary. However, the conventional point scanning confocal microscopy (PSCM) needs longer duration to acquire a full section. Moreover, the laser scans on the sample point-by-point and one-by-one, thereby result in time difference between different pixels in an image. This much reduces the temporal precision for fast events, i.e., the evoked fluorescent $\mathrm{Ca}^{2+}$ indicator hotspots in this experiment. Therefore, time resolved analysis with laser-scanning microscopes typically employs line and/or spot scans (Frank et al., 2009), thereby sacrificing spatial information. In order obtain spatial and temporal information on IHC synapses I chose to acquire the full-frame confocal images using spinning disk confocal microscopy (SDCM).

The spinning disk confocal scanner contains a Nipkow disk where has thousands of pinholes. The laser beam passes through these pinholes and accomplishes multi-pinhole scanning. The emitted fluorescence also traversed through the same pinholes and thereby confocal images can be obtained at an attached camera. The Yokogawa CSU22 which was used in this study even contains 2 Nipkow disks where the pinholes of them can almost perfectly map to each other. The pinholes of upper disk were mounted by microlens which can focus 
more laser onto the pinhole of the second disk thereby enhance the efficiency of laser passing.

While the disk is spinning, the pinholes sweep over the sample. Every 30 degrees of the scanning composes a full scanning on the samples and hence completes a full-frame image (Gräf et al., 2005). How fast the disk can spin determines the temporal resolution of the sampling. The spinning disk confocal scanner CSU22 can spin up to 5000 rounds per minutes (rpm). As mentioned, a full scanning requires the disk to spin 30 degrees, in the other words, turning one round of the disk can form 12 full-frame images. $5000 \mathrm{rpm}$ therefore can reach 1 frame per $\mathrm{ms}(1000 \mathrm{~Hz})$.

To realize the high speed acquisition, a camera with fast readout speed and low noise is necessary. Long readout time between frames would limit the sampling rate. In addition, since short exposure and imaging periods reduce the total amount of collected fluorescent light decreasing the signal, reducing the noise is critical for enabling fast imaging at acceptable signal to noise ratio. Hence the camera should have low noise, high quantum efficiency, and short readout time. The quad-chip CCD camera "NeuroCCD" consists of 4 chips, each of which composed of 20 by 20 pixels. The quantum efficiency of these chips is more than $80 \%$ at $550 \mathrm{~nm}$. The readout time can reach $40 \mu \mathrm{s}$, which only decrease the acquisition rate from $200 \mathrm{~Hz}$ to $198.4 \mathrm{~Hz}$ for the sequential images with $5 \mathrm{~ms}$ frame interval.

To complete a full scanning of a spinning disk confocal image, one pinhole should move to the position of the next pinhole, thereby ensures the complete illumination/ scanning of the samples. Therefore the frame interval of the camera should be certain constant values of time which are determined by the spinning speeds. If the frame interval is shorter than these constant durations, inhomogenous illumination can result with several parallel dark stripes in 
the image (Figure III.1). In contrast, if the frame intervals are larger than the fixed duration, it can causes parallel bright stripes because these areas are scanned one more time than others.
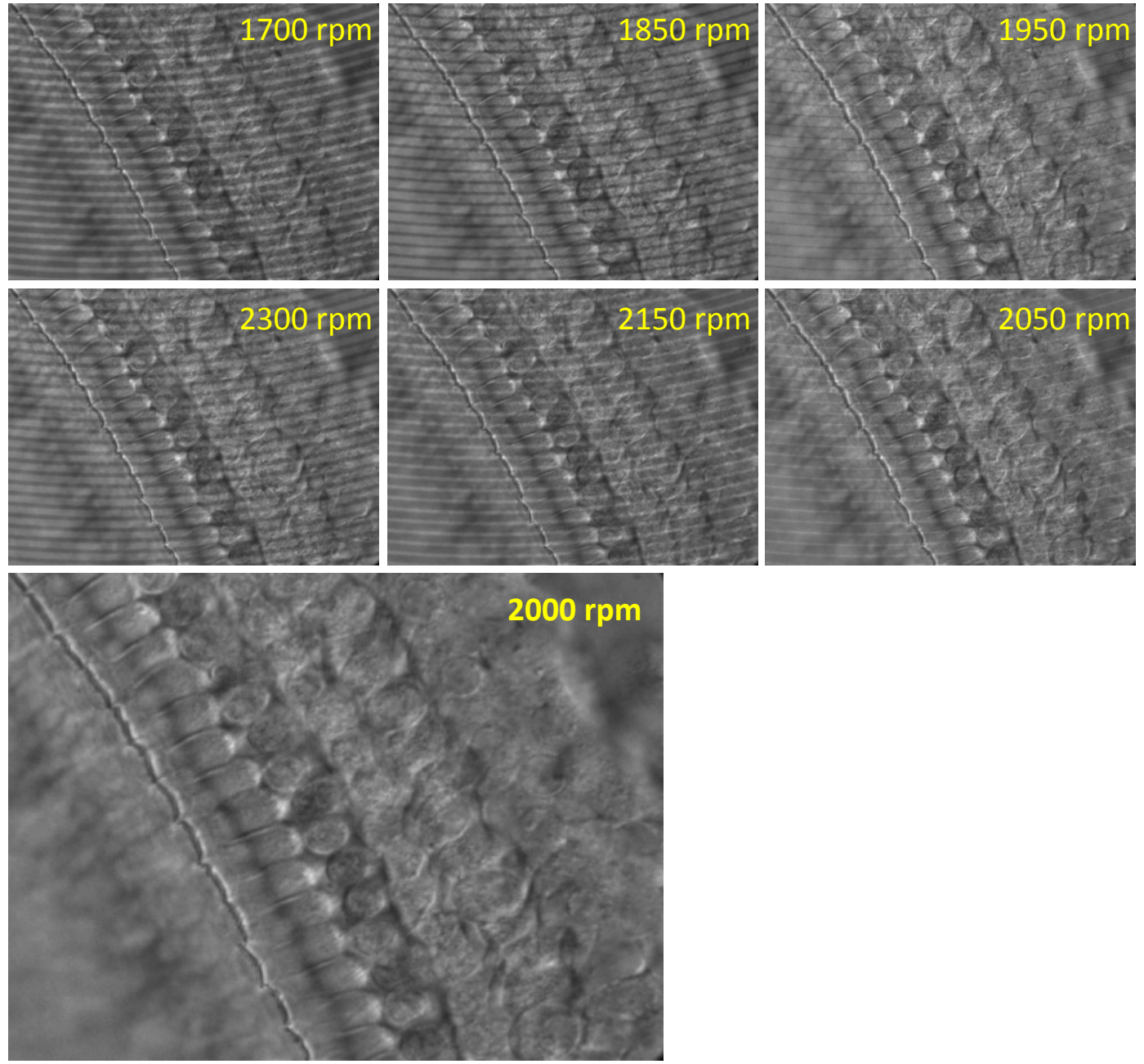

\section{Figure III.1}

\section{Non-synchronized images and the synchronized image}

This figure shows the stripes caused by the non-synchronizing of the spinning speed and the acquisition rate of the camera. In this figure, the camera acquisition rate was $5 \mathrm{~ms}$ which can be synchronized by setting the spinning speed to $2000 \mathrm{rpm}$. Top three pictures show the stripes when the spinning speed is slower than 2000 rpm, middle three pictures show the stripes when the spinning speed is faster than $2000 \mathrm{rpm}$. The bottom picture is the synchronized image. The background of these pictures is an organ of Corti.

Besides the synchronization of the camera frame rate to the spinning speed, the physical position of camera relative to the spinning disk apparatus is also crucial. In the architecture and operation of a frame-transfer CCD, the acquired image, which is integrated in the image 
array, is shifted vertically, row-by-row, to the masked storage array. However, pixels still keep on collecting photons during the vertical shift. When the movement of pinhole is parallel to this vertically, row-by-row shifting, some columns of the chip always pass through the same moving pinholes and the pixels of the column would collect photons from the same pinholes. In the end, these columns collect more photons from the pinholes than others, and result in bright stripes. In contrast, when the movement of pinholes is perpendicular to the frame shifting, pinholes sweep through several columns during frame transfer. This can also reduce the duration for a pixel to collect photons from the moving pinholes. Hence, the orientation of the frame transfer of a CCD camera should be perpendicular to the movement of pinhole. In the NeuroCCD, the $40 \mu \mathrm{s}$ frame-transfer duration is sufficiently short compared to $5 \mathrm{~ms}$ frame interval, such that we did not have to take actions towards a specific orientation.

The high speed spinning can introduce vibrations into the entire microscope and patch-clamp apparatus, compromising the image quality and potentially interfering with the patch-clamp recordings. In my experiments, I assessed the vibration by measuring the laser intensity at the output port of spinning disk scanner. Comparing to the analysis of images, the signal of the output laser does not contain the noise from camera readout, fluorescence and synchronization, which can report the noise of the disk spinning more straightly. The frequency spectrum of the output power exhibited peak frequencies in consistent with the frequencies of disk spinning (the red trace, Figure III.2B). I tried to reduce this vibration by back-mounting the scanner to the custom-made metal framework (Figure III.2A) which was fixed on the table together with the microscope. This construction mostly eliminated the oscillating signal when the spinning speed was lower than $2500 \mathrm{rpm}$ (the black trace, Figure III.2B). In figure III.2B, little coherent noise was left after the fixation. This remaining oscillating noise might arise from imperfect alignment or manufacture of these 2 spinning 
disks. A small deviation of mapping of the pinholes between two disks can result in the coherent noise of spinning frequencies because each specified mapping of pinholes only passes once by spinning per round. In spite of the fixation, it cannot totally eliminate the vibrating noise when spinning faster than $2500 \mathrm{rpm}$. The vibration even can be observed in the live imaging of samples. Therefore, in order to get the lowest noise images, I decided to use the $2000 \mathrm{rpm}$ spinning speed, which equals $400 \mathrm{~Hz}$ frame rate to acquire images.
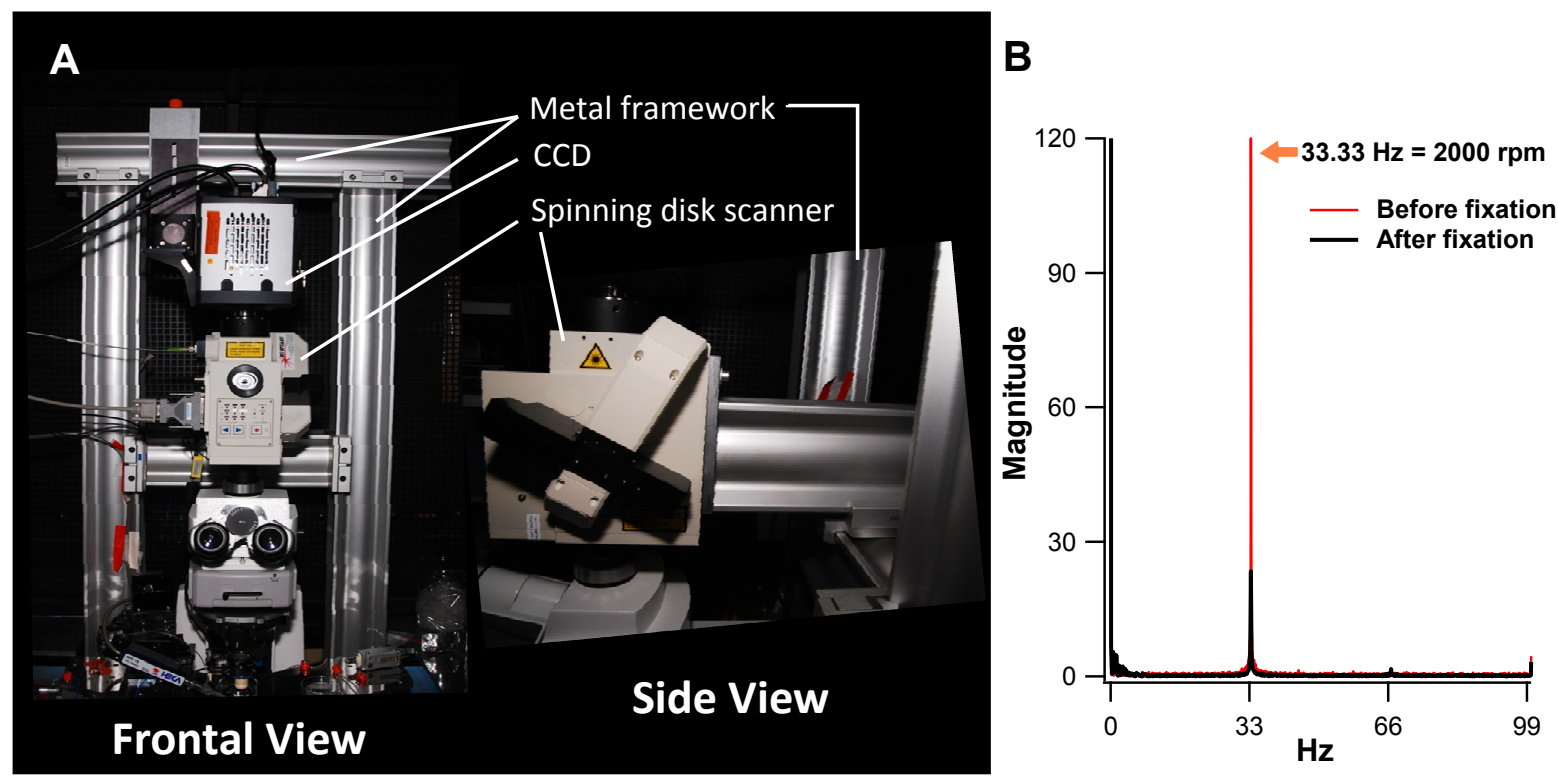

\section{Figure III.2}

\section{Mechanical construction of the microscope equipped with spinning disk scanner}

(A) shows how the setup was fixed. The left picture: frontal view of the setup. The scanner was mounted on the microscope. The right picture: side view of the setup. The scanner was back-fixed by the metal bar clamped onto the metal framework behind the setup. (B) The magnitude of fast Fourier transform (FFT) of the signal which measured the laser intensity at the output of the scanner. The spinning speed was $2000 \mathrm{rpm}$, which equals $33.33 \mathrm{~Hz}$. A peak of $33.33 \mathrm{~Hz}$ was observed in the result of FFT. The red line is the signal before fixing the setup onto the frame work. The black line represents the signal after fixation. The magnitude of the 33.33 $\mathrm{Hz}$ was greatly reduced.

\section{Observing the $\mathrm{Ca}^{2+}$ microdomain in SDCM}

In this experiment, confocal imaging was conducted simultaneously with whole-cell voltage-clamp recording. After forming a giga-seal, the IHCs patch was ruptured and the 
recording was subsequently performed. $\mathrm{Ca}^{2+}$ indicators fluo-8FF, TAMRA-conjugated, ribbon-binding peptide, and $10 \mathrm{mM}$ EGTA were perfused into the cells. The membrane potential was then clamped at certain voltages to activate the voltage-gated $\mathrm{Ca}^{2+}$ channels $\mathrm{Ca}_{v} 1.3$, thereby revealed the evoked fluorescent $\mathrm{Ca}^{2+}$ indicator hotspots. These voltage-evoked $\mathrm{Ca}^{2+}$ hotspots appeared immediately after the depolarization of the membrane potential. The diffusion distance of the incoming $\mathrm{Ca}^{2+}$ was restricted by the $\mathrm{Ca}^{2+}$ chelator EGTA. Therefore the $\mathrm{Ca}^{2+}$ indicator fluorescence should be only observed in the vicinity of $\mathrm{Ca}^{2+}$ channels. The observed $\mathrm{Ca}^{2+}$ hotspots were around 1 micrometer (Figure III.3B), therefore these $\mathrm{Ca}^{2+}$ hotspots were also called " $\mathrm{Ca}^{2+}$ microdomains". The $\mathrm{Ca}^{2+}$ microdomains almost colocalized to the labeled ribbons in my experiments (Figure III.3C). Rarely, there were very few $\mathrm{Ca}^{2+}$ hotpots observed at the places without the labeled ribbon. These result supported the previous experiments on both $\mathrm{Ca}^{2+}$ imaging and immunostaining of Ribeye and $\mathrm{Ca}_{v} 1.3 \alpha_{1 \mathrm{D}}$ (Brandt et al., 2005; Frank et al., 2009) arguing that several $\mathrm{Ca}_{v} 1.3$ were clustered nearby synaptic ribbons.

\section{$\mathrm{Ca}^{2+}$ microdomains within single IHCs vary in intensity}

Regulation of neurotransmission relies on sophisticated control of vesicular exocytosis by spatially coupled $\mathrm{Ca}^{2+}$ channels at presynaptic active zones. Presynaptic active zones are electron dense structures containing many molecules such as SNARE to regulate and operate presynaptic function (Südhof, 2012). However, functional and structural characteristics vary even for the same kind of synapses. In auditory hair cells, several kinds of the presynaptic heterogeneity have been reported. There, the size of synaptic ribbons has been shown to be positively correlated to the vesicle number of these synapses in cats (Merchan-Perez and Liberman, 1996) and frogs (Graydon et al., 2011). Additionally, $\mathrm{Ca}^{2+}$ imaging studies in the mouse cochlea have shown that the intensity of presynaptic $\mathrm{Ca}^{2+}$ 

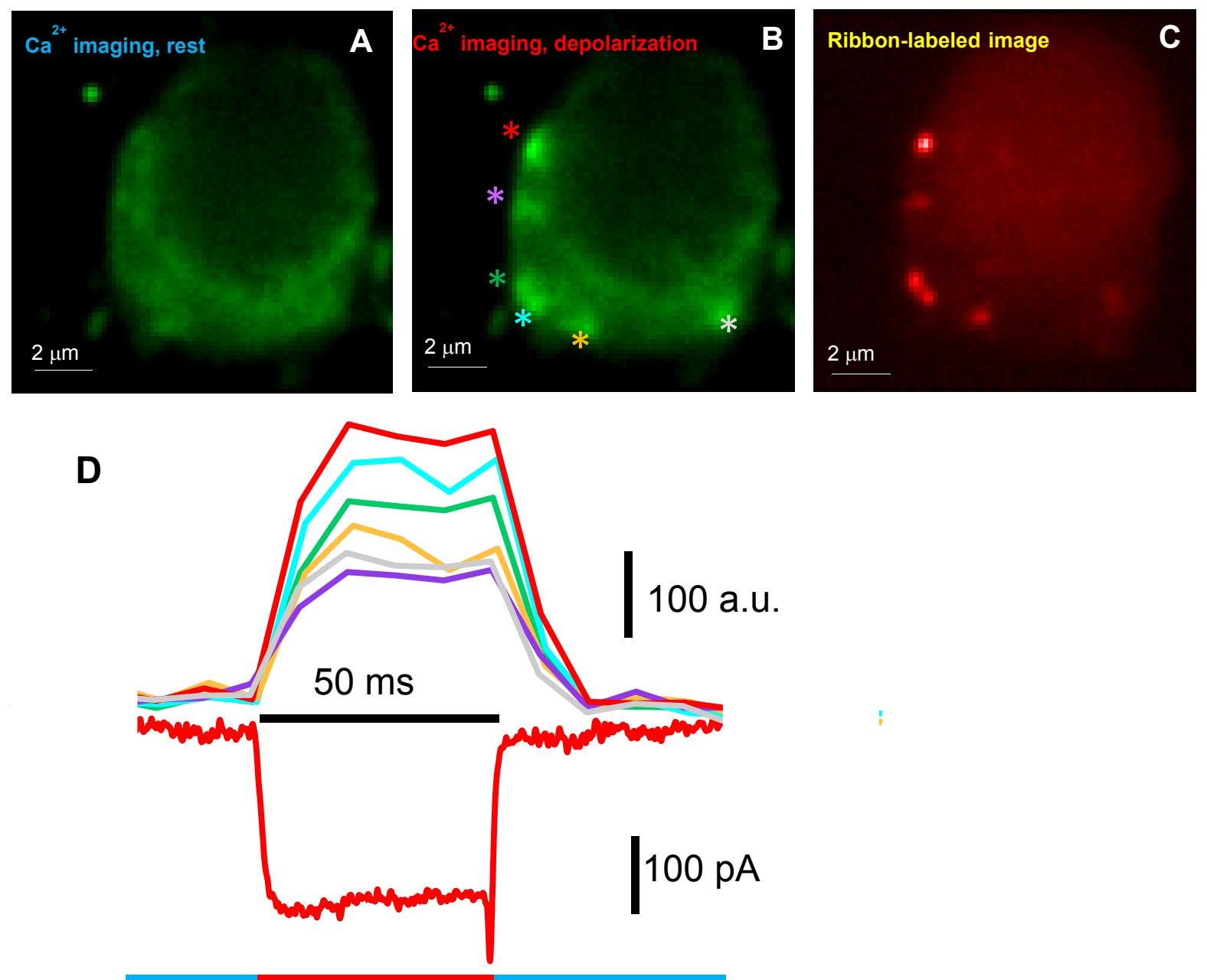

Rest Depolarization

Rest

\section{Figure III.3}

\section{Depolarization evoked $\mathrm{Ca}^{2+}$ microdomains colocalize with synaptic ribbons}

(A) A section of an IHC contained the fluorescence of $\mathrm{Ca}^{2+}$ indicators. This cell was voltage-clamped at $-87 \mathrm{mV}$. (B) The cell was then depolarized to $-7 \mathrm{mV}$. The $\mathrm{Ca}^{2+}$ hotspots were then evoked which are labeled by asterisks. Different colors of the asterisks refer to the traces in the upper half part of (D). The sizes of these hotspots range from sub-micrometer to 1 micrometer. (C) The corresponding image of (A) and $(B)$ in the channel display the stained ribbons. Notice that every $\mathrm{Ca}^{2+}$ hotspot in (B) can be assigned to a fluorescent puctum in (C). These pucta are supposed to be synaptic ribbons. (D) Upper traces demonstrate the temporal changes of the $\mathrm{Ca}^{2+}$ hotspot fluorescence. Different colors refer to different hotspots labeled in $(B)$. The lower trace shows the corresponding whole-cell ion current.

microdomains in IHCs as reported by fluorescent $\mathrm{Ca}^{2+}$ indicators display a high degree of heterogeneity (Frank et al., 2009; Meyer et al., 2009).

Interestingly, patterns of rate-level functions in different auditory neurons as well as their SR 
and acoustic threshold are quite distinctive. It is interesting to investigate whether the properties of presynaptic evoked-Ca ${ }^{2+}$ elevation determine the acoustic threshold of postsynaptic auditory neurons. Here, I applied SDCM to record the depolarization-evoked $\mathrm{Ca}^{2+}$ influx and resulting fluorescent intensity of $\mathrm{Ca}^{2+}$ microdomains in patch-clamped IHCs. In comparison with a previous study (Frank et al., 2009) which acquired images of $\mathrm{Ca}^{2+}$ microdomains by means of the PSCM, SDCM is able to acquire a whole image of a confocal section in shorter time (10 ms and shorter vs. typically $100 \mathrm{~ms}$ per frame in SDCM). The SDCM enabled us to characterize all synapses in less time and with shorter duration of stimulation and therefore to assess $\mathrm{AZ}$ size, $\mathrm{Ca}^{2+}$ microdomain maximal intensity and voltage-dependence. The $\mathrm{Ca}^{2+}$ microdomains usually resided in 10 15 $\mu \mathrm{m}$ of the basal part of IHCs. To reveal these $\mathrm{Ca}^{2+}$ microdomains, images were acquired in every confocal section in the basal part and in each section the cells were depolarized and fluorescence changes were recorded. The scanned regions were first determined by scanning the labeled ribbons by searching for the upper and lower bound of the ribbon-exhibiting sections. The $\mathrm{Ca}^{2+}$ imaging was subsequently performed. Maximal presynaptic $\mathrm{Ca}^{2+}$ influx was evoked by $50 \mathrm{~ms}$ long depolarization from $-87 \mathrm{mV}$ to $-7 \mathrm{mV}$ (Figure III.3D). The fluorescence change of the central strongest pixel $\left(\Delta \mathrm{F}_{\max }\right)$ in $\mathrm{x}, \mathrm{y}$, and $\mathrm{z}$, and of the mean of 9 pixels in $\mathrm{x}$ and $\mathrm{y}$ surrounding the central strongest pixel $\left(\Delta \mathrm{F}_{\mathrm{avg}}\right)$, were used to assess the strength of $\mathrm{Ca}^{2+}$ microdomains. Normalized intensities of fluorescence increase, $R_{\max }\left(\Delta F_{\max } / F_{0}\right)$ and $R_{\text {avg }}$ $\left(\Delta \mathrm{F}_{\mathrm{avg}} / \mathrm{F}_{0}\right)$, were also used to avoid errors of fluorescent intensity caused by variance of dye concentration or incomplete loading of the cell.

The distribution of the fluorescence intensities of all $\mathrm{Ca}^{2+}$ hotspots from 28 cells showed large variability (figure III.4A). The coefficients of variation (C.V.) of $\Delta F_{\text {avg }}$ and $R_{\text {avg }}$ were 0.70 and 0.59 , respectively. The C.V. of $\Delta \mathrm{F}_{\mathrm{avg}}$ is in accordance with previous findings (Frank et al., 2009). However, the distribution of $F_{0}$ of each spot also showed considerable variability (C.V. 
$=0.37$, figure $1.1 \mathrm{~B}$ ) and the variability of $F_{0}$ existed within individual cells too (figure III.4D). Hence, instead of $\Delta F_{\text {avg }}, R_{\text {avg }}$ was preferred to estimate the intensity of $\mathrm{Ca}^{2+}$ hotspots.

The heterogeneity of $\mathrm{F}_{0}$ might result from variance in the loading of dye or in washing out of endogenous $\mathrm{Ca}^{2+}$ buffers among hair cells. Therefore, I also inspected the mean and standard deviation (S.D.) of every hair cell (Figure III.4C, D). The C.V. of $\Delta \mathrm{F}_{\text {avg }}$ and $\mathrm{R}_{\text {avg }}$ in individual cells ranged from 0.14 to 1.0 for the $\Delta F_{\text {avg }}$ and 0.15 to 0.81 for the $R_{\text {avg }}$ (table III.1). The grand averages of the mean $\Delta \mathrm{F}_{\text {avg }}$ and $R_{\text {avg }}$ of all examined cells were $124.4 \pm 58.9$ a.u. and $0.72 \pm 0.25$, respectively. These results further confirmed that $\mathrm{Ca}^{2+}$ microdomains exhibit a heterogeneous intensity both within individual hair cells as well as between different hair cells. 

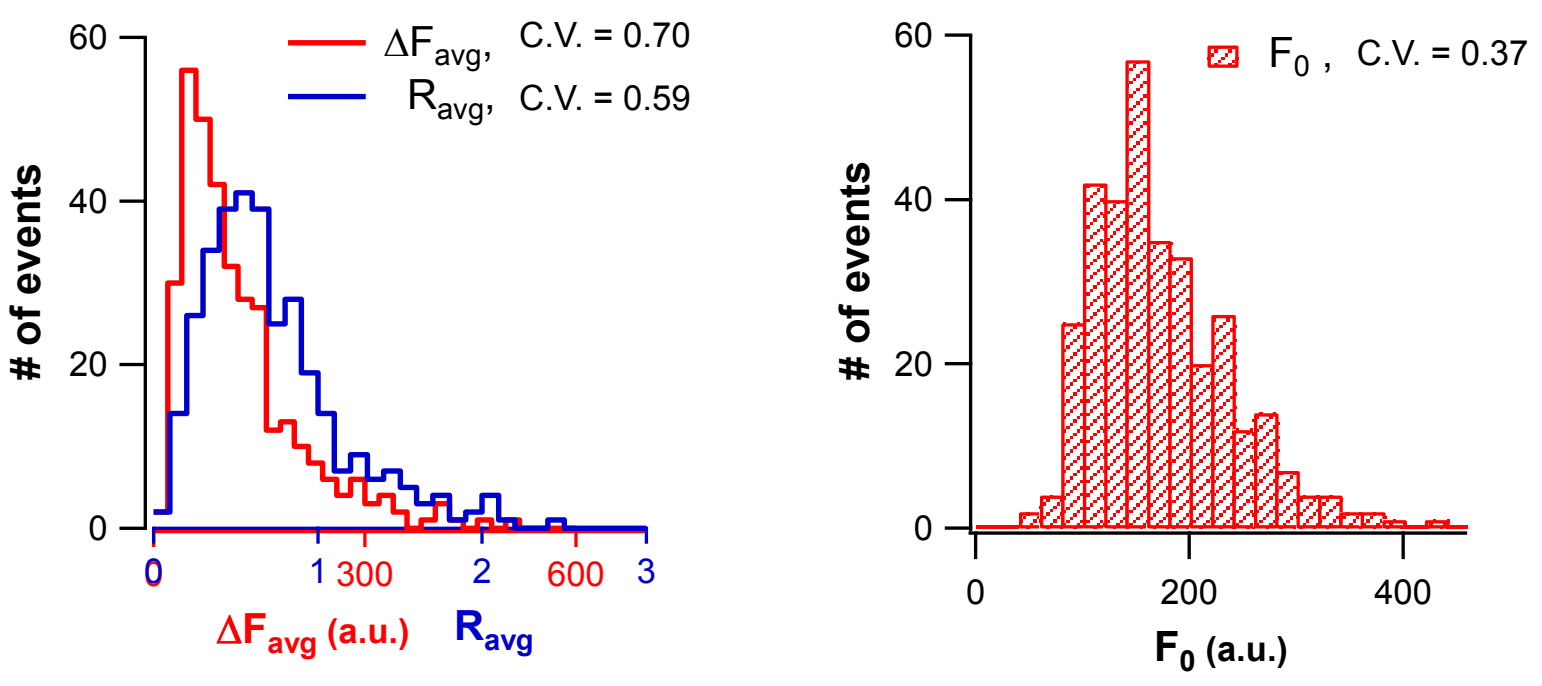

C
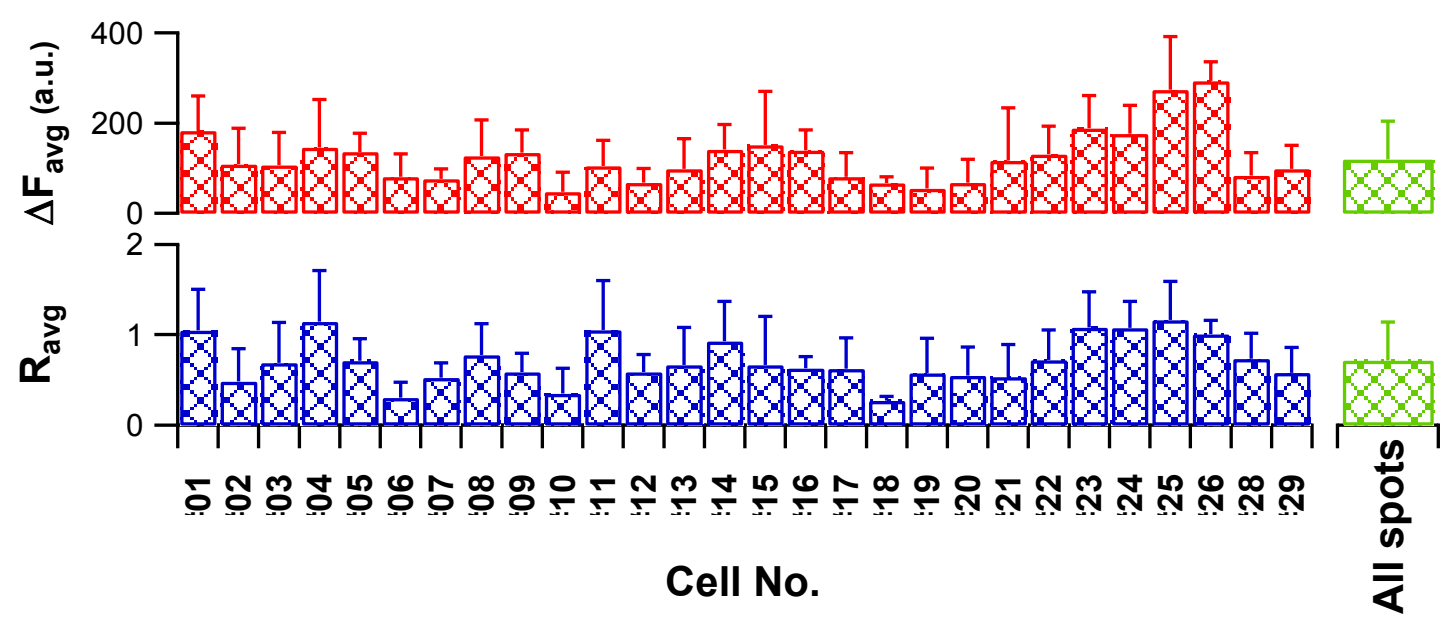

D

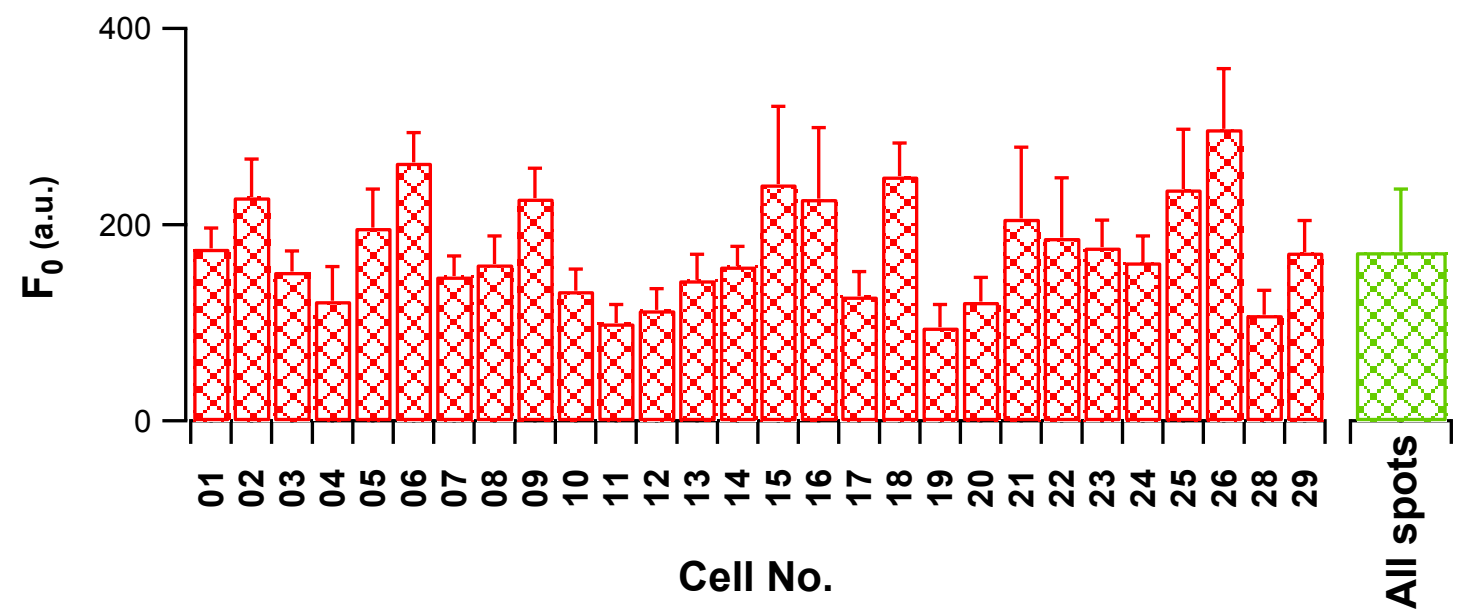




\section{Figure III.4}

Variable intensity of hotspots of $\mathrm{Ca}^{2+}$ indicator fluorescence

(A) Distribution of $\Delta \mathrm{F}_{\text {avg }}$ and $\mathrm{R}_{\text {avg }}$ of all examined $\mathrm{Ca}^{2+}$ microdomains. Both variables show a wide distribution with a C.V. of 0.70 and 0.59 , respectively. (B) A certain heterogeneity is also apparent in the distribution of the individual microdomains' background fluorescence at resting potential $F_{0}$ (C.V. of 0.37). (C), (D) Those histograms display the mean $+S D$ of individual cells' $\Delta F_{\text {avg }}$ (red bars, $C$ ) and $R_{\text {avg }}$ (blue bars, $C$ ) as well as $F_{0}$ (D). The green bars show the average of all examined $\mathrm{Ca}^{2+}$ microdomains.

\section{Table III.1}

Summary of the average intensities of $\mathrm{Ca}^{2+}$ microdomains in all measured cells

\begin{tabular}{|c|c|c|c|c|c|c|}
\hline Cell & Nr. of spots & $\mathrm{I}_{\mathrm{Ca}}(\mathrm{pA})$ & $\Delta \mathrm{F}_{\mathrm{avg}}$ & C.V. of $\Delta F_{\text {avg }}$ & $\mathrm{R}_{\mathrm{avg}}$ & C.V. of $R_{\text {avg }}$ \\
\hline \#01 & 14 & "-214.2 & "182.61 & 0.42 & 1.05 & 0.44 \\
\hline \#02 & 14 & -394.6 & 108.33 & 0.74 & 0.48 & 0.75 \\
\hline \#03 & 13 & -297.7 & 106.81 & 0.68 & 0.69 & 0.64 \\
\hline \#04 & 15 & -149.6 & 145.79 & 0.72 & 1.14 & 0.49 \\
\hline \#05 & 7 & -104.4 & 135.72 & 0.31 & 0.71 & 0.34 \\
\hline \#06 & 14 & -340.7 & 81.22 & 0.62 & 0.30 & 0.56 \\
\hline \#07 & 14 & -243.9 & 76.40 & 0.29 & 0.53 & 0.32 \\
\hline \#08 & 12 & -178 & 126.92 & 0.63 & 0.78 & 0.44 \\
\hline \#09 & 13 & -170.5 & 134.51 & 0.37 & 0.59 & 0.35 \\
\hline \#10 & 10 & -151.6 & 48.24 & 0.89 & 0.35 & 0.81 \\
\hline$\# 11$ & 15 & -233.6 & 104.80 & 0.54 & 1.05 & 0.52 \\
\hline \#12 & 12 & -187.2 & 67.92 & 0.46 & 0.59 & 0.33 \\
\hline$\# 13$ & 16 & -202.3 & 98.20 & 0.69 & 0.67 & 0.62 \\
\hline \#14 & 12 & -166.5 & 141.51 & 0.39 & 0.93 & 0.47 \\
\hline \#15 & 10 & -142.3 & 151.89 & 0.78 & 0.66 & 0.81 \\
\hline$\# 16$ & 13 & -174.9 & 139.97 & 0.32 & 0.63 & 0.20 \\
\hline \#17 & 9 & -163.9 & 80.58 & 0.66 & 0.63 & 0.54 \\
\hline \#18 & 6 & -130.2 & 66.96 & 0.21 & 0.27 & 0.18 \\
\hline \#19 & 11 & -168.6 & 54.77 & 0.82 & 0.58 & 0.67 \\
\hline \#20 & 12 & -203 & 68.16 & 0.75 & 0.55 & 0.58 \\
\hline \#21 & 14 & -187.6 & 116.48 & 1.00 & 0.53 & 0.67 \\
\hline \#22 & 11 & -157.8 & 130.30 & 0.48 & 0.72 & 0.45 \\
\hline \#23 & 11 & -231.9 & 188.67 & 0.39 & 1.08 & 0.37 \\
\hline \#24 & 6 & -110.1 & 176.24 & 0.35 & 1.08 & 0.27 \\
\hline \#25 & 12 & -205.4 & 274.34 & 0.43 & 1.16 & 0.37 \\
\hline$\# 26$ & 7 & -161 & 293.51 & 0.14 & 1.00 & 0.15 \\
\hline \#28 & 14 & -180.2 & 83.96 & 0.61 & 0.74 & 0.38 \\
\hline$\# 29$ & 14 & -223.6 & 98.74 & 0.53 & 0.58 & 0.49 \\
\hline Mean & 12 & -195.55 & 124.41 & 0.54 & 0.72 & 0.47 \\
\hline
\end{tabular}




\section{Voltage dependence of activation varies among different $\mathrm{Ca}^{2+}$ microdomains}

In addition to the $\mathrm{Ca}^{2+}$ microdomain intensity, in this study I also investigated the voltage-activation properties of $\mathrm{Ca}^{2+}$ microdomains. $\mathrm{Ca}^{2+}$ currents mediated by different alternative splice variants of the $\alpha_{1 D}$ subunit of $\mathrm{Ca}_{v} 1.3$ have been shown to vary in their voltage dependence of activation (Singh et al., 2008; Juhasz-Vedres et al., 2011; Tan et al., 2011b). If an IHC synapse contains an isoform of $\mathrm{Ca}_{v} 1.3$ that activates at more negative voltages, weaker stimulation/sound intensity is capable to evoke neurotransmission from this synapse. I argue that the voltage-dependence of synaptic $\mathrm{Ca}^{2+}$ influx determines the rate-level function of the postsynaptic spiral ganglion neurons. Heterogeneity in the voltage dependence of $\mathrm{Ca}^{2+}$ influx between the synapses of a given $\mathrm{IHC}$ is then a plausible candidate mechanism for causing the different spontaneous rates and acoustic sensitivities of SGNs, which are evident from comparing their rate-level functions. To investigate the heterogeneity of the voltage-dependent activation among the presynaptic $\mathrm{Ca}_{v} 1.3$ channel clusters the voltage-dependent activation of all individual $\mathrm{Ca}^{2+}$ microdomains was examined by fast confocal 3D Ca ${ }^{2+}$-imaging in IHCs. Since numerous repeated depolarizations of IHCs can deteriorate the health of IHCs, it is problematic to apply a complete step-IV protocol on every confocal section through a cell. Instead, I adopted a ramp-IV protocol to evoke the $\mathrm{Ca}^{2+}$ influx instead of the step-IV. Unlike the step-IV protocols, in the ramp-IV protocol, full activation of the $\mathrm{Ca}^{2+}$ microdomains could be acquired in $100 \mathrm{~ms}$ within a single depolarization protocol, which greatly reduced the overall amount of stimulation.

The membrane potential was ramped from $-87 \mathrm{mV}$ to $+13 \mathrm{mV}$ within $100 \mathrm{~ms}$ (Figure III.5A). One drawback of the ramp-IV protocol is the requirement for the ramping speed to accommodate the time needed for the gating of ion channels. Therefore, it is necessary to confirm that the used ramping speed is adequate to achieve activation like that obtained in 
the steady state current during depolarizing steps. If the ramping speed is too fast, the number of opening channels at a given voltage would be underestimated, especially for low depolarizations because of the slow activation rate at these potentials. In figure III.5B, the current-voltage (IV) and FV relationships acquired with voltage ramp were compared to those of the step depolarization. The FV trace overlapped largely between both voltage protocols. The IV trace also overlapped well before $-10 \mathrm{mV}$ whereas the IV from step depolarizations had larger inward (negative) current than the ramp IV. This difference of the ion currents between two protocols might attribute to the contribution of other ions such as $\mathrm{Cs}^{+}$and $\mathrm{K}^{+}$. Nevertheless, the overlapping of both IV and FV traces before $-10 \mathrm{mV}$, where more than $90 \%$ of the $\mathrm{Ca}^{2+}$ current is usually activated, ensured sufficient time for the voltage-dependent activation of the $\mathrm{Ca}_{v} 1.3$ in this ramp protocol $(1 \mathrm{mV} / \mathrm{ms})$. Therefore, we used a speed of $1 \mathrm{mV} / \mathrm{ms}$ for the subsequent experiments.

Figure III.5A demonstrates the experimental protocol and an example of recorded FV and IV traces. They were subsequently divided by the traces which estimate the full activated current/fluorescence by extrapolate the linear portion between $0 \mathrm{mV}$ to $+20 \mathrm{mV}$ in the $\mathrm{FV}$ or IV trace to every corresponding voltage and thereby obtain their fractional activation traces. (See material and method for details). These fractional activation trace were fitted by Boltzmann equation to estimate two fitting parameters, the half-maximal activation voltage $\left(\mathrm{V}_{0.5}\right)$ and the slope-factor $(\mathrm{k})$.

The comparison of average fractional activation curves for current and $\mathrm{Ca}^{2+}$ indicator fluorescence is displayed in figure III.5C. The activation properties of $\mathrm{Ca}^{2+}$ microdomains (225 $\mathrm{Ca}^{2+}$ microdomains in 21 cells) differed than those of the whole cell $\mathrm{Ca}^{2+}$ currents of the same cells. The $\mathrm{V}_{0.5}$ of $\mathrm{Ca}^{2+}$ microdomains was $-23.6 \mathrm{mV}$, which was about $6 \mathrm{mV}$ more positive than that of the $\mathrm{Ca}^{2+}$ current $(-29.8 \mathrm{mV})\left(\mathrm{P}_{\alpha}<0.001\right)$. The slope factor $(\mathrm{k})$ of $\mathrm{Ca}^{2+}$ 
microdomains was about $1 \mathrm{mV}$ lower than whole cell $\mathrm{Ca}^{2+}$ current $(6.7 \mathrm{mV}$ for hotspots, 7.5 for current, $\mathrm{P}_{\alpha}<0.001$ ) (Figure III.5C). Furthermore, voltage-activation of $\mathrm{Ca}^{2+}$ microdomains was more variable, the standard deviation of $\mathrm{V}_{0.5}$ and $\mathrm{k}$ were \pm 4.2 and $\pm 1.2 \mathrm{mV}$, respectively in comparison with \pm 2.3 and $\pm 0.5 \mathrm{mV}$ in the activation of $\mathrm{Ca}^{2+}$ currents (Figure III.5D,E). Since the whole cell current simply discloses the response of all $\mathrm{Ca}^{2+}$ channels in an $\mathrm{IHC}$, the jitter of the $V_{0.5}$ of ion current among cells is expected to be lower than that of $V_{0.5}$ of the individual $\mathrm{Ca}^{2+}$ microdomains. I conclude that there is substantial variability in the voltage-dependence of $\mathrm{Ca}^{2+}$ current activation among the active zones of an IHC.

In order to inspect whether the maximal intensity of $\mathrm{a} \mathrm{Ca}^{2+}$ microdomain is correlated with its voltage-dependence of activation, I related the $\mathrm{V}_{0.5}$ and $\mathrm{k}$ acquired in the ramp-IV protocol (Figure III.5A) to the baseline-normalized fluorescence change in the $\mathrm{Ca}^{2+}$ microdomain center in response to step depolarization to -7 measured in the experiment of figure III.4. $-7 \mathrm{mV}$ instead of the maximum intensity of depolarization (around $-17 \mathrm{mV}$ ) was used to ensure the full activation of $\mathrm{Ca}^{2+}$ channels at the active zone. As shown in figure III.5F and III.5G, the $\mathrm{V}_{0.5}$ and $\mathrm{k}$ of the strong $\mathrm{Ca}^{2+}$ microdomains were mainly close to the mean of the population, whereas weaker ones had higher or lower $\mathrm{V}_{0.5}$ and $\mathrm{k}$.

The relation of $V_{0.5}$ and $k$ is displayed in figure $111.5 \mathrm{H} . V_{0.5}$ and $k$ exhibited a moderate positive correlation (correlation coefficient: 0.47 ). This result indicates that active zones that activate at the most negative $V_{0.5}$ also display the steepest voltage dependence of activation, suggesting that they drive the most sensitive synapses. 
A
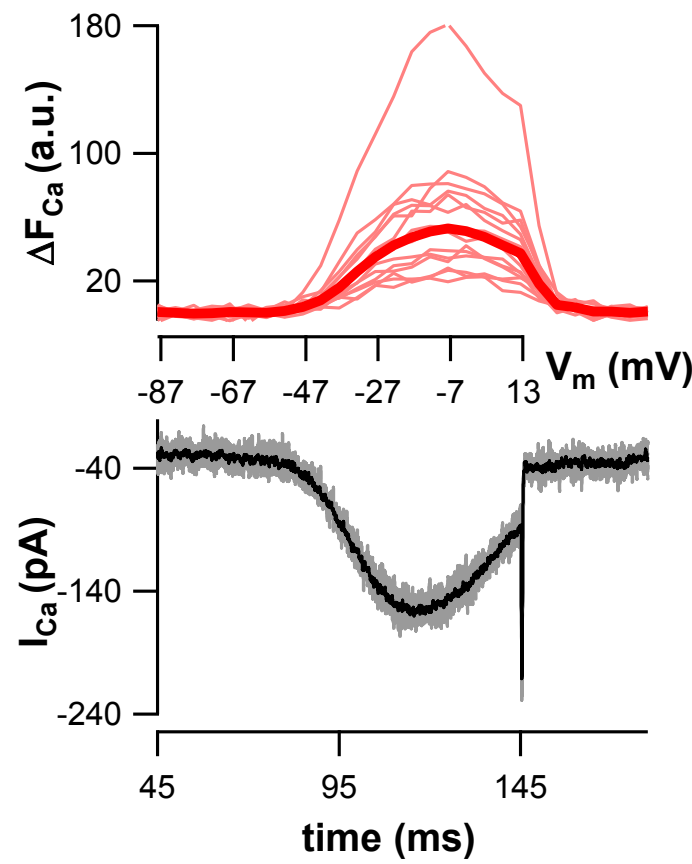

C

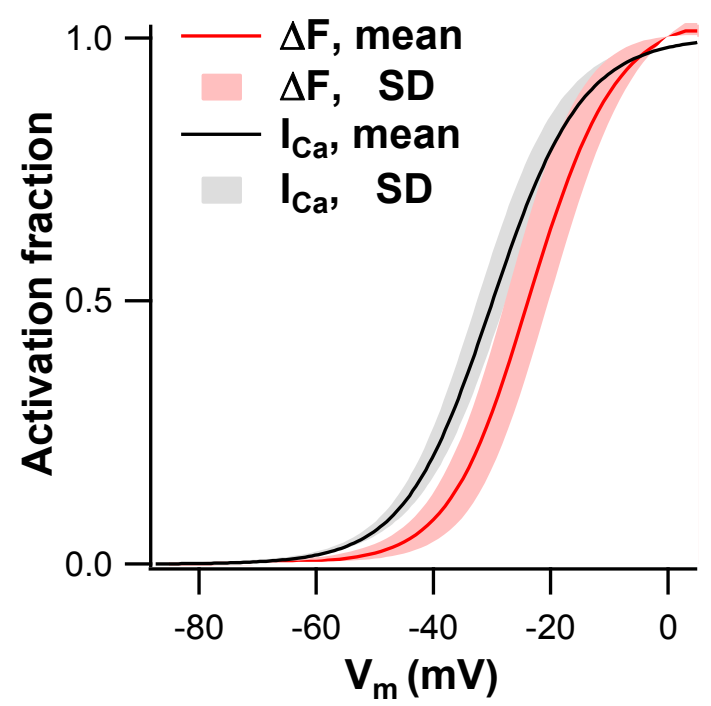

B

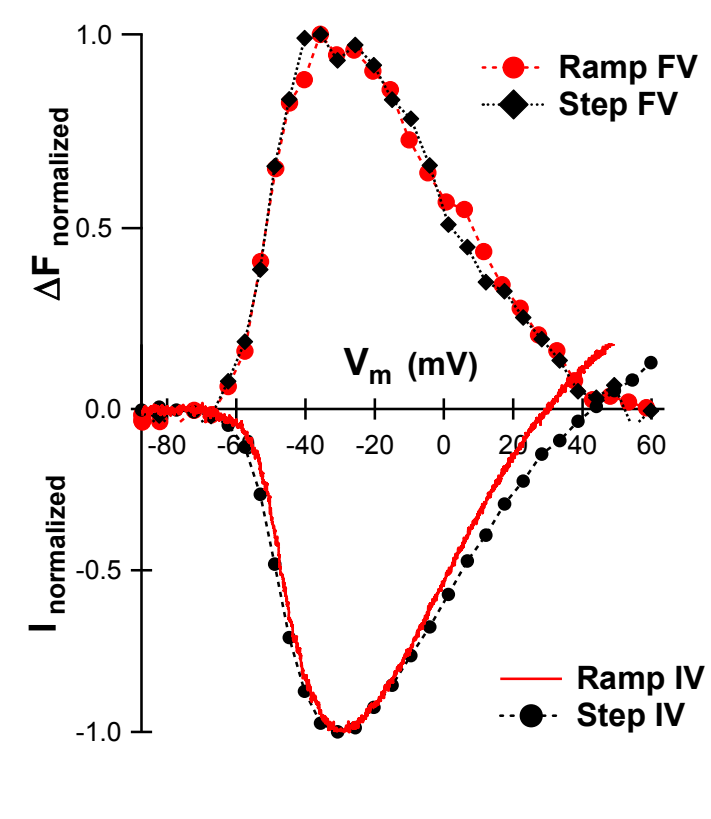


D

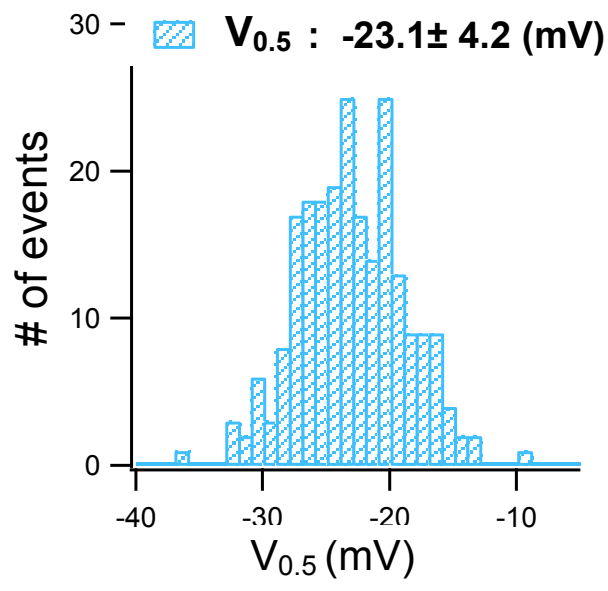

F

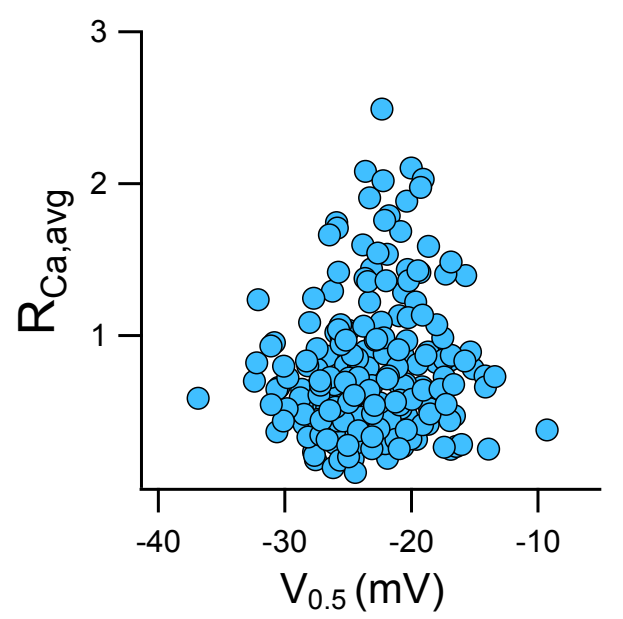

E

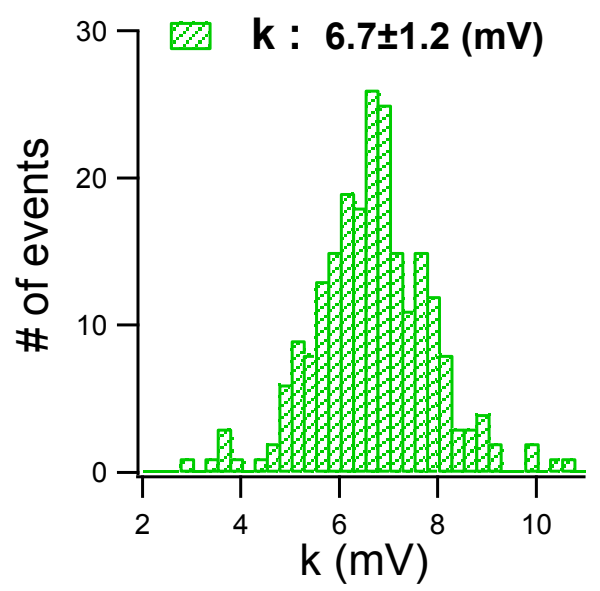

G

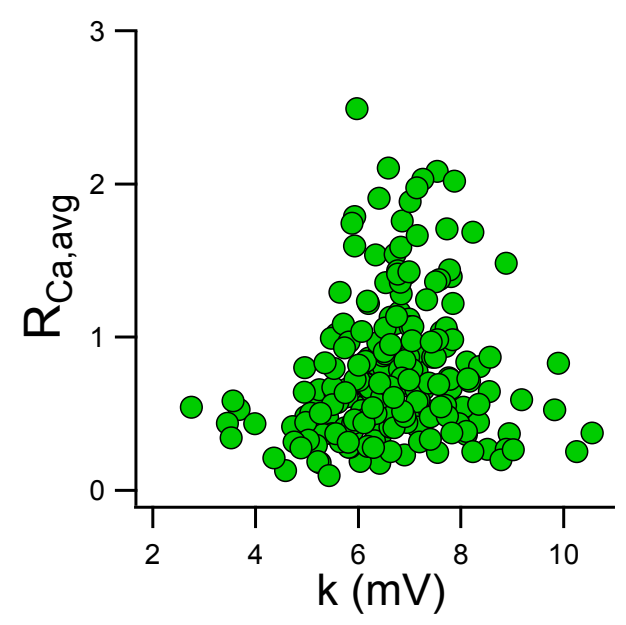

H

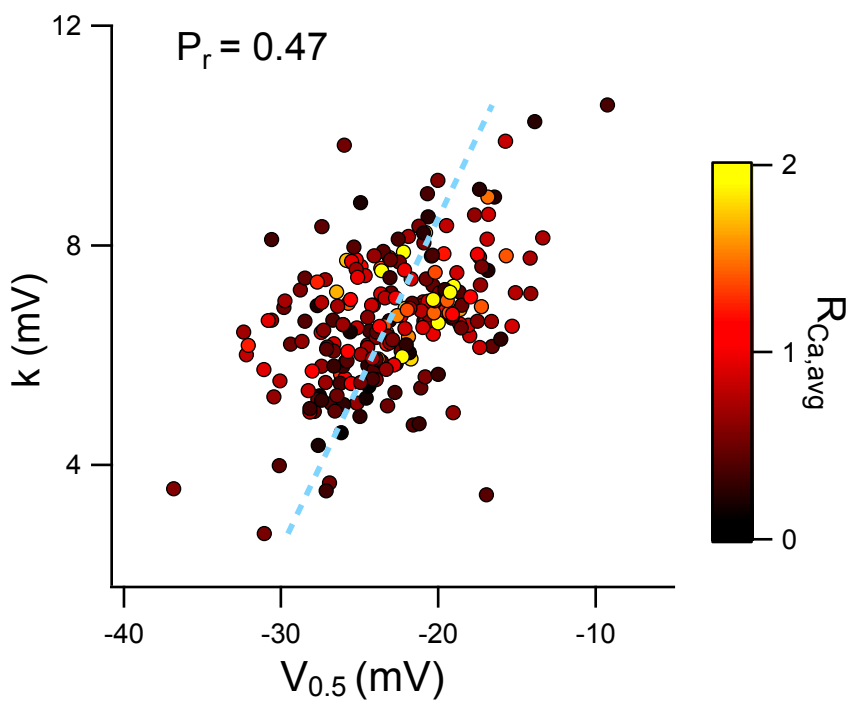




\section{Figure III.5}

\section{Properties of the activation of $\mathrm{Ca}^{2+}$ microdomains}

$\mathrm{Ca}^{2+}$ microdomains were activated by the ramp depolarization from $-87 \mathrm{mV}$ to $+13 \mathrm{mV}$ in $100 \mathrm{~ms}$, and the images were acquired at a frame rate of $200 \mathrm{~Hz}$ (5 ms exposure per frame). (A) Demonstration of a representative cell. The grey and light red traces represent the individual $\mathrm{Ca}^{2+}$ currents and $\mathrm{Ca}^{2+}$ fluorescence traces (of the individual hotspots), and the black and red traces are the respective averages. (B) Comparison of the IV and FV traces of step and ramp depolarization protocol. Both the ramp and step depolarized the cell from -87 to $+63 \mathrm{mV}$. The voltage step size in step protocol was $5 \mathrm{mV}$, and the frame rate of ramp protocol was $200 \mathrm{~Hz}$. (C) Black and red traces are the mean IV from 21 cells and FV from 225 hotspots, respectively. Both $\mathrm{V}_{0.5}$ and $\mathrm{k}$ of the activation of $\mathrm{Ca}^{2+}$ microdomain were significantly different to the $\mathrm{Ca}^{2+}$ current $\left(\mathrm{P}_{\alpha}<0.001\right)$. The shaded areas represent the standard deviation of IV and FV. The detailed distribution of $\mathrm{V}_{0.5}$ and $\mathrm{k}$ are shown in (D) and (E). (F), (G) Plots of the relationship between $\mathrm{V}_{0.5}$ and $\mathrm{k}$ to intensity of $\mathrm{Ca}^{2+}$ microdomains, $\mathrm{R}_{\text {avg }}$. The intensities of $\mathrm{Ca}^{2+}$ microdomains were taken from the step depolarization which is shown on figure III.4. $(\mathbf{H})$ Plot of $\mathrm{V}_{0.5}$ vs. $\mathrm{k}$ for all $\mathrm{Ca}^{2+}$ microdomains showing a moderate linear correlation with the correlation coefficient $\mathrm{P}_{\mathrm{r}}=0.47$. The color codes for the intensity of $\mathrm{Ca}^{2+}$ microdomains. 


\section{Table III.2}

Summary of the activation analysis in all individual cells

Columns " $\mathrm{V}_{0.5}$ " and " $\mathrm{k}$ " show the average of the $\mathrm{V}_{0.5}$ and $\mathrm{k}$ of within a cell. Notice that the value in "Nr of Spot" is different from table III.1 because in the activation experiments, some spots could not be detected owing to dimmer signal and shorter frame acquisition time (5 ms).

\begin{tabular}{|c|cccccc|}
\hline Cell & Nr. Of Spots & $\mathrm{R}_{\text {avg }}$ & $\mathrm{V}_{0.5}(\mathrm{mV})$ & S.D. of $\mathrm{V}_{0.5}$ & $\mathrm{k}(\mathrm{mV})$ & S.D. of $\mathrm{k}$ \\
\hline \hline$\# 01$ & 14 & 1.05 & -19.53 & 2.50 & 6.57 & 0.59 \\
$\# 02$ & 10 & 0.48 & -25.86 & 2.12 & 6.37 & 0.80 \\
$\# 03$ & 13 & 0.69 & -25.94 & 1.41 & 5.47 & 0.38 \\
$\# 04$ & 14 & 1.14 & -22.79 & 2.30 & 7.39 & 0.52 \\
$\# 05$ & 6 & 0.71 & -21.64 & 2.62 & 6.45 & 0.94 \\
\hline$\# 06$ & 14 & 0.3 & -24.47 & 1.78 & 5.67 & 0.60 \\
$\# 08$ & 12 & 0.78 & -24.28 & 2.87 & 6.40 & 0.65 \\
$\# 10$ & 3 & 0.35 & -18.77 & 1.87 & 7.42 & 0.61 \\
$\# 11$ & 13 & 1.05 & -24.77 & 2.09 & 6.20 & 1.08 \\
$\# 12$ & 12 & 0.59 & -26.68 & 3.58 & 7.39 & 1.17 \\
\hline$\# 14$ & 12 & 0.93 & -18.50 & 3.04 & 6.52 & 0.58 \\
$\# 15$ & 6 & 0.66 & -18.68 & 1.40 & 7.83 & 0.83 \\
$\# 17$ & 13 & 0.63 & -23.27 & 2.37 & 6.68 & 0.82 \\
$\# 18$ & 5 & 0.27 & -19.02 & 5.51 & 7.78 & 1.96 \\
$\# 19$ & 9 & 0.58 & -17.83 & 2.46 & 6.30 & 1.92 \\
\hline$\# 20$ & 8 & 0.55 & -19.83 & 5.66 & 6.86 & 2.05 \\
$\# 21$ & 14 & 0.53 & -25.17 & 2.51 & 7.35 & 0.95 \\
$\# 23$ & 10 & 1.08 & -21.22 & 3.08 & 7.22 & 0.38 \\
$\# 25$ & 12 & 1.16 & -21.27 & 3.12 & 7.18 & 0.75 \\
$\# 28$ & 12 & 0.74 & -30.87 & 2.65 & 5.91 & 1.88 \\
\hline$\# 29$ & 13 & 0.58 & -23.70 & 2.87 & 6.56 & 1.27 \\
\hline
\end{tabular}




\section{Heterogeneous size of synaptic ribbons in single inner hair cell}

The structure of synapses has been found to be related to their physiological performance (Holderith et al., 2012). Moreover, Calcium channels are highly colocalized with the presynaptic ribbon structure (Brandt et al., 2005; Frank et al., 2010). It's would be interesting to investigate the relationship between ribbon sizes and their strength of depolarization-evoked $\mathrm{Ca}^{2+}$ microdomains. Indeed, in a previous study the fluorescence intensity of peptide-bound synaptic ribbons, which were utilized to approximate the size of ribbon, was shown to vary among active zones (C.V. of approximately 0.7$)$ and to correlate with strength of the $\mathrm{Ca}^{2+}$ signal (Frank et al., 2009), suggesting that the strength of $\mathrm{Ca}^{2+}$ microdomains increases with the number of $\mathrm{Ca}^{2+}$ channels at the larger active zones. To further test this hypothesis, we also used a similar ribbon reporter (TAMRA-conjugated C-terminal binding protein 2 (CtBP2) binding peptide) to identify the ribbons and approximate their size in ruptured-whole cell patch clamp recordings and performed confocal $\mathrm{Ca}^{2+}$ imaging as described above. CtBP2 is the major component of synaptic ribbons (Schmitz et al., 2000; Uthaiah and Hudspeth, 2010b). Larger ribbons are supposed to have more CtBP2 thereby should bind more peptides. Therefore, it is reasonable to estimate the size of ribbons by measuring the intensities of the fluorescent puncta which were revealed by ribbon-binding peptides. These puncta were further confirmed to be synaptic ribbons by their corresponding evoked $\mathrm{Ca}^{2+}$ microdomains. The benefit of this method is that peptide labels the ribbon in the living cell in comparison with immunohistochemistry or electron microscopy, which needs to fix tissues. However, due to the diffraction-limited resolution of confocal microscopy the structure of the small IHC ribbons could not be investigated.

Figure III.6A demonstrates an image of a cell filled with the TAMRA-conjugated CtBP2-binding peptide. The bright puncta represent synaptic ribbons. Both measured 
intensity $\left(F_{\text {ribbon }}\right)$ of fluorescence and the background-normalized intensity $\left(F_{\text {ribbon }} / F_{\text {nearby }}\right)$ of fluorescence were used to estimate the intensity of ribbon staining. Pixels which were 2 to 3 pixels (around $500 \mathrm{~nm}$ ) away from the border of visible brighter puncta toward the intracellular direction were averaged (the left arrowhead in figure III.6A) as a background for individual staining puncta. In figure III.6B, the fluorescence intensity of labeled ribbons is displayed versus their corresponding background fluorescence $\left(F_{\text {nearby }}\right)$, which was positively correlated $F_{\text {nearby. }}$. Hence, the fluorescence intensity of the ribbon was divided by their background to obtain the normalized intensity $\left(F_{\text {ribbon }} / F_{\text {nearby }}\right)$.

Figure III.6C and III.6E showed the population distribution of 217 spots from 19 cells. Both the distribution of $F_{\text {ribbon }}$ and $F_{\text {ribbon }} / F_{\text {nearby }}$ displayed strong heterogeneity, but still less than that of $\mathrm{Ca}^{2+}$ microdomain (figure III.4A). This heterogeneity also exists within single cells (figure III.6D,F). The extent of heterogeneity was different among cells, some cells had strong heterogeneity (\#21, C.V. is 0.64 in $D$ and 0.48 in $E$ ), but there were also less heterogeneous cells, of which the C.V. were not larger than 0.2 (\#24 in III.6D). 
A

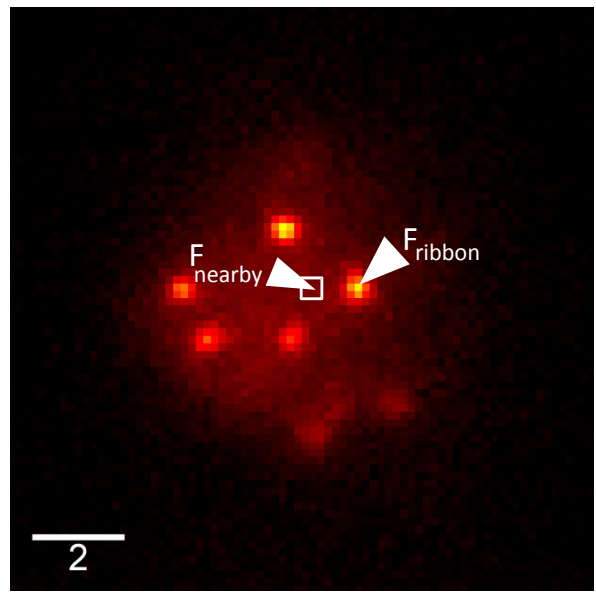

C

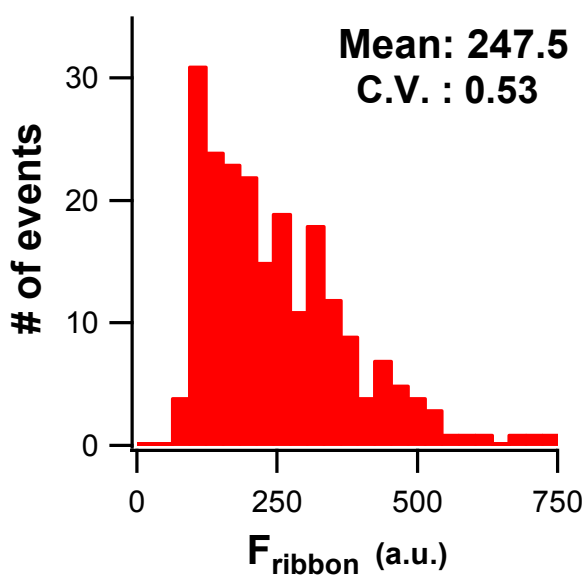

E

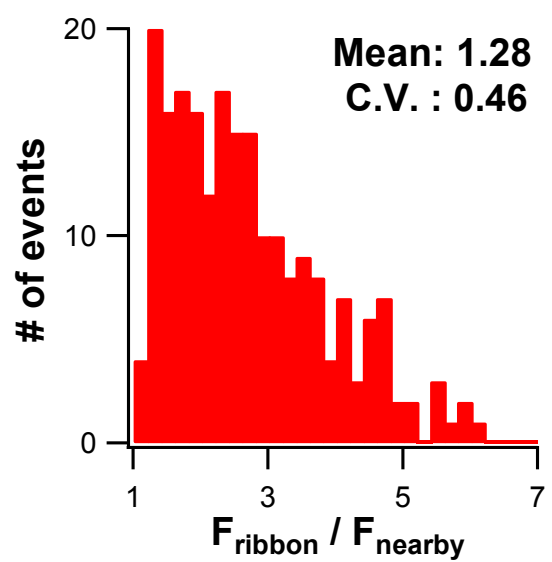

B

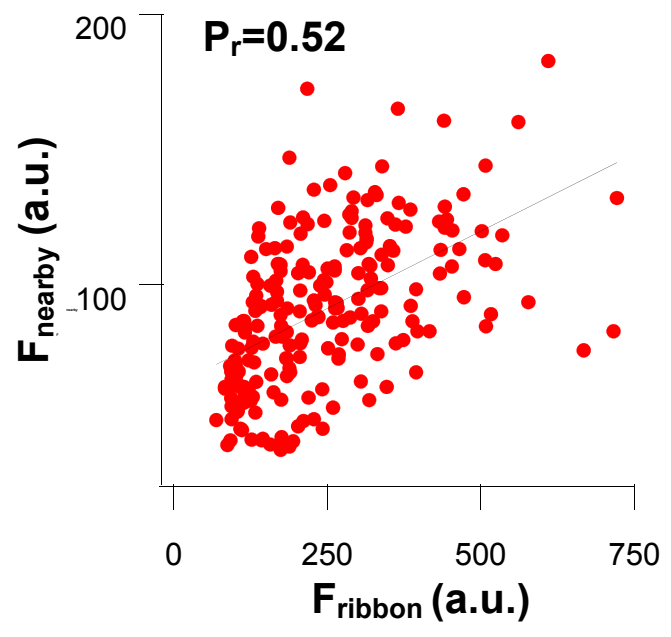

D

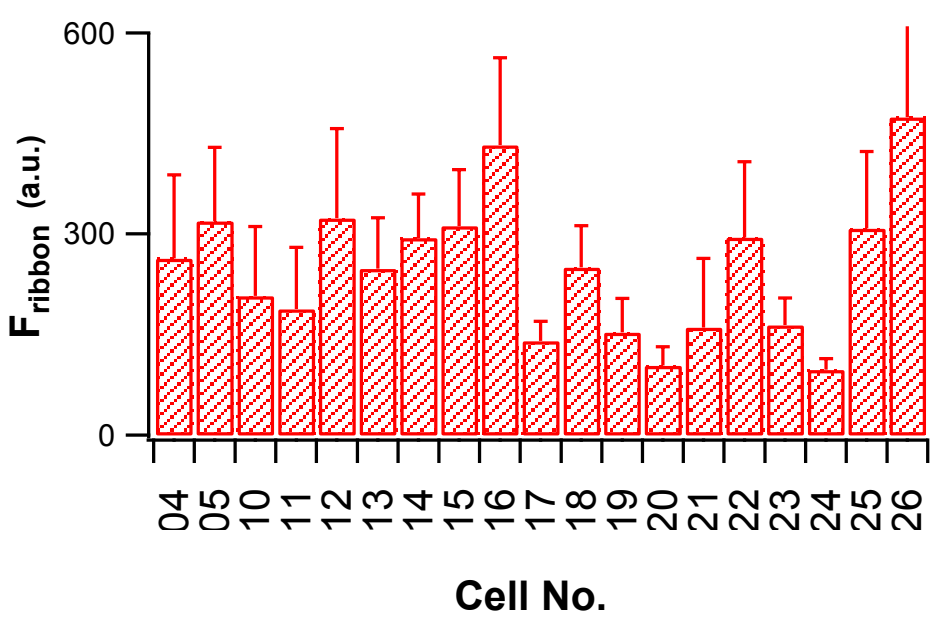

F

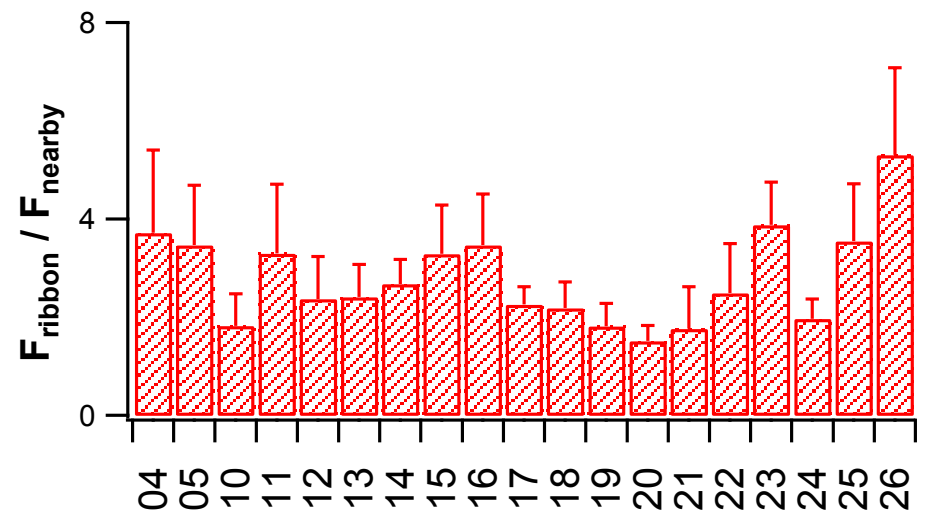

Cell No. 


\section{Figure III.6}

\section{Population properties of the ribbon fluorescence}

(A) Demonstration of one sample image of TAMRA-conjugated CtBP2-binding peptide. The brighter spots identified here are recognized as synaptic ribbons. The intensity of ribbon staining was picked up from the intensity of brightest pixel in the center of every individual punctum $\left(\mathrm{F}_{\text {ribbon }}\right)$, which is pointed by right arrowhead. Every punctum was specified a reference background region containing 9 pixels, which is pointed out by the left arrowhead. These background regions were located 2 to 3 pixels away from the visible area of puncta in the direction toward the center of the cell. The average of these 9 pixels was taken as the background intensity

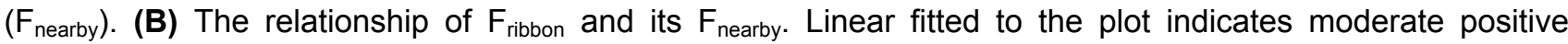
relationship with correlation coefficient $P_{r}=0.52$. (C), (E) The distribution of measured $\left(F_{\text {ribbon }}\right)$ and the normalized $\left(F_{\text {ribbon }} / F_{\text {nearby }}\right)$ intensity of ribbons across 217 puncta. (D),(F) show the mean+S.D. of ribbon puncta in 19 cells.

\section{Positive correlation of ribbon fluorescence and $\mathrm{Ca}^{2+}$ microdomain intensity}

The intensities of $\mathrm{Ca}^{2+}$ microdomains were compared to the fluorescence of the corresponding ribbons in order to investigate the relation of the strength of $\mathrm{Ca}^{2+}$ microdomains with the size of corresponding synaptic ribbons. Because other $\mathrm{Ca}^{2+}$ indicators were reported to bind the presynaptic dense body in frog hair cells (Issa and Hudspeth, 1996), it is crucial to inspect whether the $\mathrm{Ca}^{2+}$ indicator Fluo-8FF also gathered on presynaptic synaptic ribbons of mouse IHCs. Figure III.7A displays the correlation of the

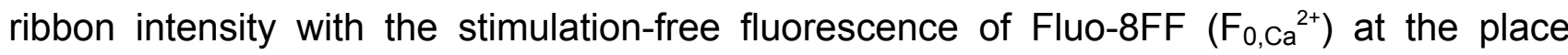
where the $\mathrm{Ca}^{2+}$ microdomains appeared. The positive correlation indicates that the resting Fluo-8FF fluorescence was unevenly distributed probably due to binding of $\mathrm{Ca}^{2+}$ indicator to the synaptic ribbon. Other possible explanation includes the position of focus plane in the cell. Illumination on the apical plane can collect more photons because the longitudinal point spread function of excitation light excited fluorophores in both upper and lower juxta-focal planes. On the other hand, in the basal end of hair cell, the upper juxta-focal plane contained fluorophores but lower juxta-focal plane did not, so it resulted to weaker cumulative intensity 
of emission light. To further examine these ideas, I compared the $F_{0}$ to the $F_{\text {nearby }}$ (Figure III.7B), which was defined in figure III.6. In figure III.7B the weak positive correlation of $\mathrm{F}_{0, \mathrm{Ca}}{ }^{2+}$ to $\mathrm{F}_{\text {nearby }}$ implied that the possible mechanism of the uneven $\mathrm{F}_{0}$ might result from the binding of $\mathrm{Ca}^{2+}$ indicators to the synaptic ribbons. Certainly, to avoid this problem, the normalized $\Delta \mathrm{F} / \mathrm{F}_{0}$ was preferred to be used to estimate the intensity of $\mathrm{Ca}^{2+}$ hotspots.

Figure III.7C-F demonstrate the relationship of ribbon fluorescence and the background-normalized intensity of the $\mathrm{Ca}^{2+}$ microdomains $\left(\mathrm{R}_{\mathrm{avg}}, \mathrm{R}_{\max }\right)$. Both the measured intensity $\left(F_{\text {ribbon }}\right)$ (Figure III.7C,E) and normalized intensity $\left(F_{\text {ribbon }} / F_{\text {nearby }}\right)$ (Figure III.7D,F) of ribbon were compared to $R_{\text {avg }}$ and $R_{\max }$. The $R_{\text {avg }}$ was more strongly positive correlated to $F_{\text {ribbon }} / F_{\text {neraby }}$ (correlation coefficient $\left.\left(P_{r}\right)=0.55\right)$ than to the measured intensity $F_{\text {ribbon }}\left(P_{r}=\right.$ 0.33). I further checked the $P_{r}$ in all individual cells. The red dots in figure III.7C to $E$ are the synapses of the cell with strongest positive correlation and the blue dots are those of the weakest positive-correlated cells. Among all of the cells, only one cell was observed to exhibit negative correlation between the intensity of the $\mathrm{Ca}^{2+}$ microdomain and that of the stained ribbons, all others showed positive correlation, although the correlation was variable among cells. $\mathrm{Ca}^{2+}$ microdomains always resided on the border of a cell. The fluorescent point spread functions of the excitation light might excite the volume inside and outside of a cell. Hence, the averaged intensity $\mathrm{R}_{\mathrm{avg}}$ might underestimate real intensities of $\mathrm{Ca}^{2+}$ microdomains, therefore the intensities of central pixel $\left(R_{\max }\right)$ were compared too. As shown in panels $E$ and $F$, the result was similar to that found with $R_{a v g}$ (panels $C$ and $D$ ).

Regarding the positive correlation shown in figure III.6B, the result of $F_{\text {ribbon }} / F_{\text {nearby }}$ was favored to be used because it might avoid the plane effect mentioned above. In conclusion, I propose that the larger ribbons might have stronger $\mathrm{Ca}^{2+}$ microdomains probably due to the presence of more $\mathrm{Ca}^{2+}$ channels. 
A

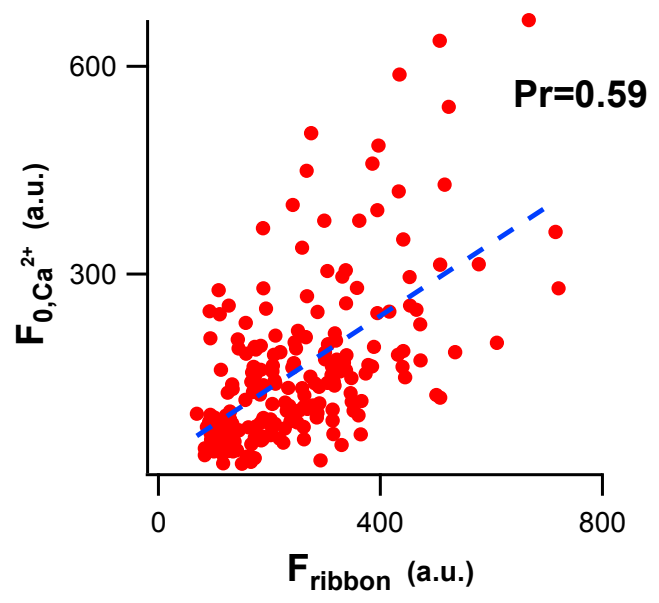

C

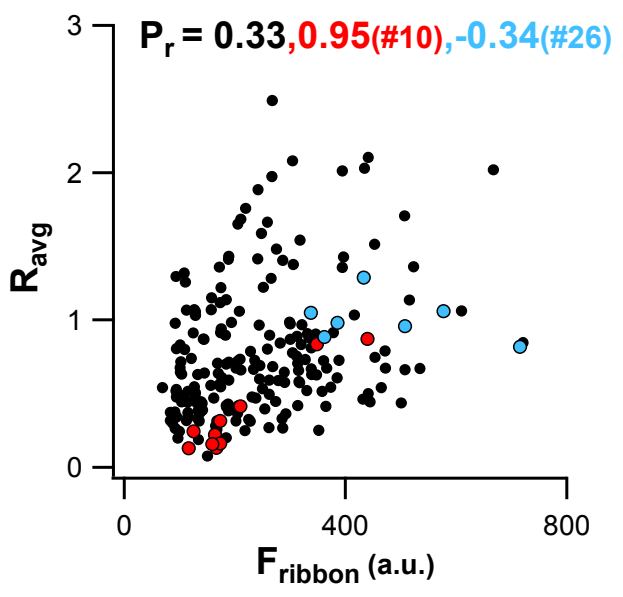

E

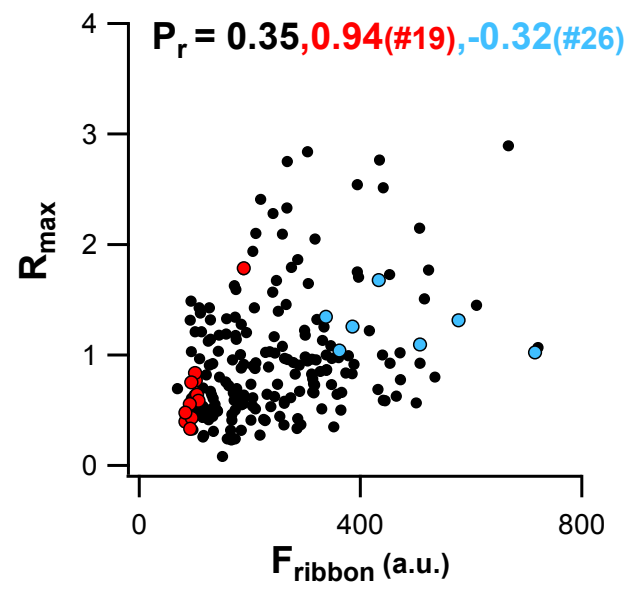

B

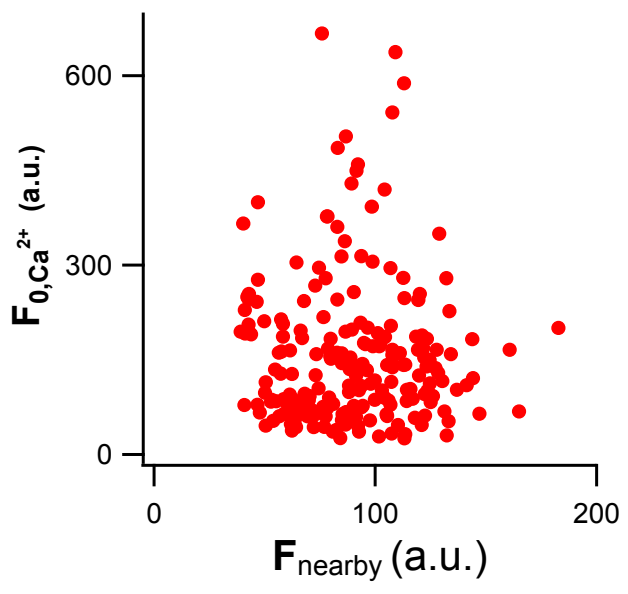

D

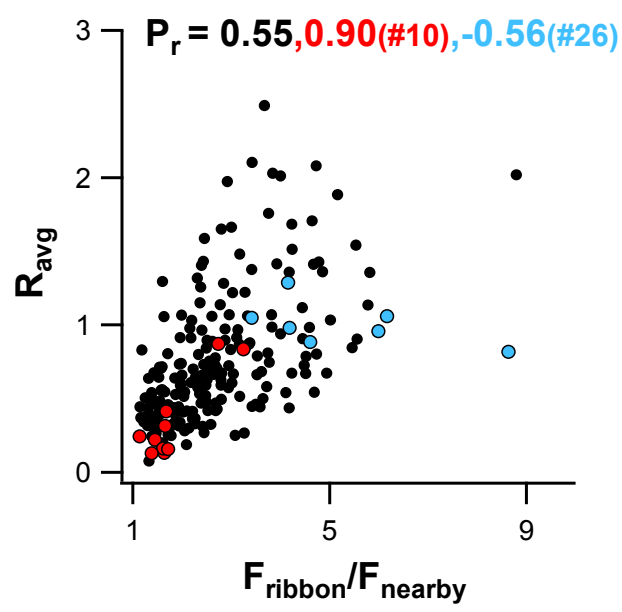

F

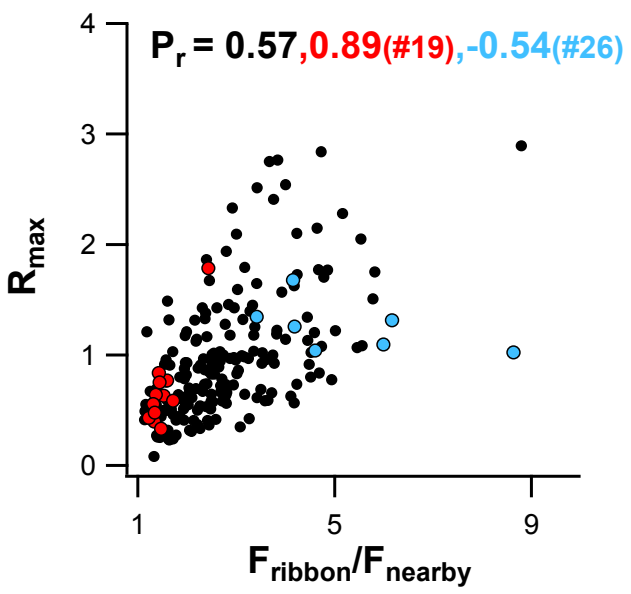




\section{Figure III.7}

\section{Relationship of the "size" of synaptic ribbons and the corresponding $\mathrm{Ca}^{2+}$ microdomains}

(A) The relationship of intensity of ribbons and their respective $\mathrm{Ca}^{2+}$ microdomains before depolarization. The fluorescence intensity $\left(\mathrm{F}_{0, \mathrm{Ca}}{ }^{2+}\right)$ of the region where $\mathrm{Ca}^{2+}$ microdomains reveal was positive correlated with the intensity of the labeled ribbon $\left(F_{\text {ribbon }}\right)$ with $P_{r}=0.59$, whereas $(B)$ the relationship with nearby background fluorescence $\left(F_{\text {nearby }}\right)$ did not display any obvious correlation with $F_{0, \mathrm{Ca}}{ }^{2+}\left(P_{r}=-0.05\right)$. (C) and (D) The relationship of $\mathrm{R}_{\text {avg }}$ of $\mathrm{Ca}^{2+}$ microdomains with related ribbon staining. The fluorescent intensity of stained ribbon was estimated by measured intensity $F_{\text {ribbon }}$ in $(C)$ and normalized intensity $F_{\text {ribbon }} / F_{\text {nearby }}$ in (D). The red points and text demonstrate every hotspots and correlation coefficient from strongest positive correlative cell among all. The light blue points and text demonstrate the weakest positive-correlating cell among all. (E) and $(F)$ show the same concept of $(C)$ and $(D)$ instead that the normalized maximum intensity of $\mathrm{Ca}^{2+}\left(\mathrm{R}_{\max }\right)$ was taken rather than the normalized mean intensity.

\section{The properties of microdomains and ribbons exhibit polar gradient}

Functional and structural heterogeneity extensively exist in hair cell afferent synapses. Measuring the response of the SGNs to sound amplitude in vivo and their spontaneous action potential firing rate also manifested various characteristics. Interestingly, Liberman indicated the SR of auditory nerves changed around the circumstance of IHCs (Liberman, 1982; Merchan-Perez and Liberman, 1996). He also pointed out that the auditory nerve fibers with larger caliber tended to have high SR (Liberman, 1982) and the high SR fibers were further reported to have lower auditory threshold for sound-evoked AP firing. One might think that this phenomenon could be reasonably explained by cable theory: a thicker fiber has higher conductance to efficiently transmit the depolarizing potential, thereby has lower failure rate of AP firing. However, both the first seminodes of Ravier located nearby the IHCs and the small membrane area (Hossain et al., 2005) of the postsynaptic bouton (Rutherford et al., 2012) enable that the AP can fire proximal to the IHCs. Therefore, the caliber of fiber might not be the most important factor to determine the efficiency of AP firing. On the other hand, forward masking experiments indicated that the availability of readily 
releasable vesicles at the IHC AZ influences the efficiency of AP firing (Harris and Dallos, 1979; Spassova et al., 2004). The variability of the auditory threshold as well as SR may be releated to the heterogeneity of evoked $\mathrm{Ca}^{2+}$ microdomains. Different characteristics of $\mathrm{Ca}^{2+}$ microdomains could result in different sensitivities of synaptic transmission to the membrane potential changes thereby contribute the various auditory thresholds. Based on the observed hair cell polarity of $S R$, it is certainly feasible to examine whether the heterogeneous $\mathrm{Ca}^{2+}$ microdomains have the spatial preference around the IHC.

The primary goal of my $\mathrm{PhD}$ work was to study the position-dependence of synapse properties in functional imaging experiments. In case I found spatial gradients, these should be compared to previous findings on position-dependent active zone morphology and functional properties of auditory nerve fibers (see introduction). While direct optical assays of transmitter release or postsynaptic firing have not yet been established, I argue that imaging the presynaptic $\mathrm{Ca}^{2+}$ signal is instrumental, because its results can be interpreted within the straight forward framework of stimulus-secretion coupling at this synapse (Brandt et al., 2005; Frank et al., 2010).

To understand the position-dependence of the synapse in IHCs, the positions of every synapse should be first determined. The circumferential position of every IHC thereby should be clarified in order to assign the orientations of the IHCs. According to the circumferential positions, the IHC can be partitioned by 2 orthogonal axes, the modiolar-pillar axis and the tonotopical axis whereby the polarities "modiolar side", "pillar side" or "tonotopical apical", "tonotopical basal" side are given to the cell. This definition was widely used in previous studies (Liberman, 1982; Merchan-Perez and Liberman, 1996; Meyer et al., 2009). By means of this definition, the position of the synapses can be demonstrated as a polar chart in cylindrical coordinates because of the cylinder-like basal 
part of the cell. The advantage of this polar demonstration is that it can faithfully represent these "modiolar-pillar" and "tonotopical apical-basal" polarity by the coordinate of angle.

To follow this definition of cell's position, the shape of every IHC is required to describe the relative position of every $\mathrm{Ca}^{2+}$ microdomain/synaptic ribbon inside a cell. Therefore, after the acquisition of all evoked $\mathrm{Ca}^{2+}$ microdomains, the morphologies of IHCs were depicted by the cytosolic fluorescence of TAMRA-conjugated ribbon-staining peptides. Comparing to the previous studies where the organ of Corti was fixed and mechanically constrained by the coverslip in immunostaining experiments, observing the morphologies of alive $\mathrm{IHCs}$ during patch-clamp can provide both physiological and morphological information, and it might also better preserve the original shapes of IHCs.

The main difficulty to define the cell's geometry in this study is that the membrane of the patched IHCs was affected by the patch pipette to some extent (pulling or twisting). In addition, during the cleaning procedure of the organ of Corti before patch-clamp, removal of supporting cells could also cause drifting, shifting or twisting IHCs, which changed the relative circumferential position of these IHCs in the tissue. Therefore, the cell's geometry could not be easily and generally assumed. Therefore, I established a new procedure to define the geometry based on the morphology of individual patched IHCs. The basic concept of this method is to find the "plane of symmetry" which can divide the 3D images of acquired IHCs to two mapping images. Because this plane was supposed to be perpendicular to the alignment of the stereocilia, the normal vector of this "plane of symmetry" $\left(\mathrm{V}_{\text {sym }}\right)$ (Figure III.8A) can be used to be the surrogate of the tonotopical axis.

To transfer the acquired images from canonical Cartesian coordinate into self-defined cylindrical coordinate, 3 orthogonal reference axes and one reference point are required to 
be defined. After obtaining the plane of symmetry, the second axis and the reference point were assigned on this plane. The contacting outline of the pillar cell to IHC was then used to be second axis $\left(V_{z}\right)$ (Figure III.8B). Because this line lay on the plane of symmetry, it was orthogonal to $\mathrm{V}_{\text {sym }}$. Then the $\mathrm{IHC}$ was resampled into sequential parallel sections along the axis of the $V_{z}$. Then the center of mass of the largest fluorescent section (Figure III.8B) in the resample images was assigned as the center point (reference point) of the cell. The third axis $V_{m p}$ (Figure III.8C) was then the cross product of $V_{\text {sym }}$ and $V_{z}$. By $V_{z}, V_{s y m}, V_{m p}$, and the center point, the Cartesian $(x, y, z)$ coordinate can be transferred to cylindrical $(r, \theta, h)$ coordinate where $r$ represents the radius of the synapse, $\theta$ represents the polarity as described above, and $\mathrm{h}$ represents the altitudinal position of the synapses (Figure III.8C).
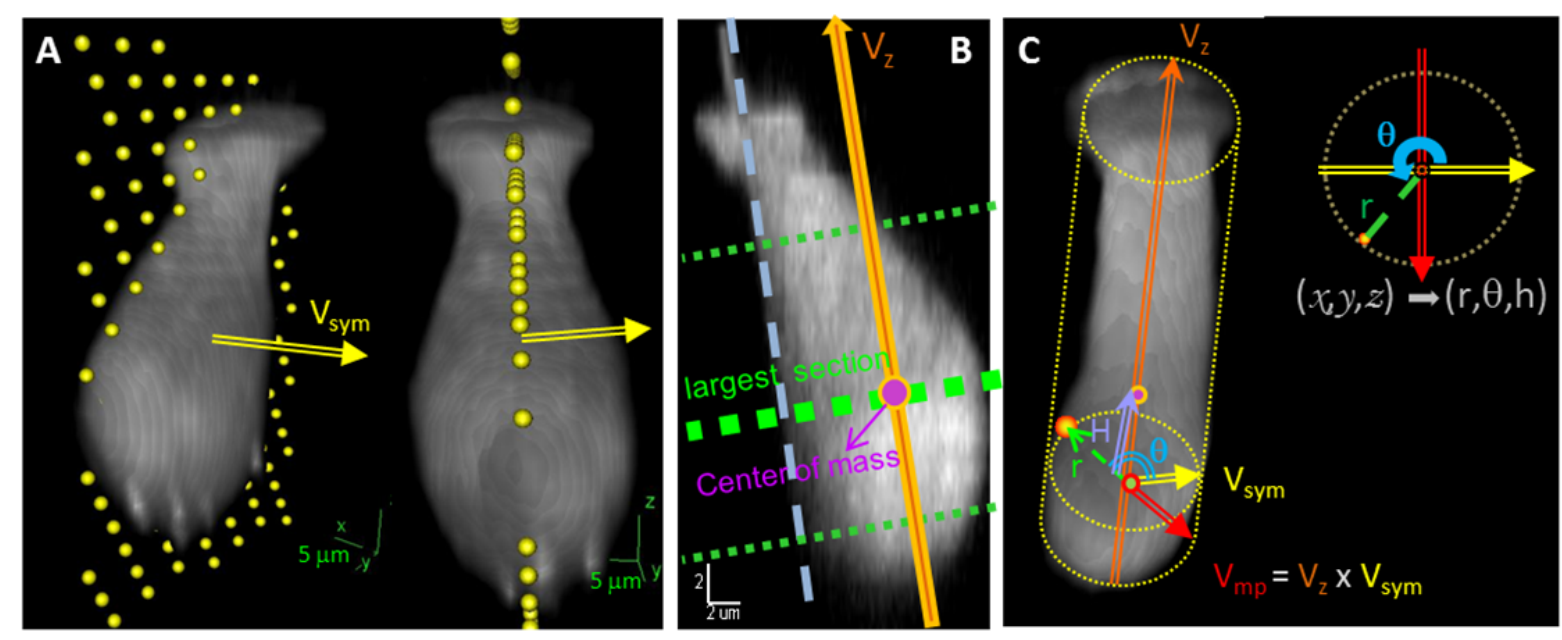

\section{Figure III.8}

\section{The definition of the cylindrical coordinate within IHCs}

(A) The dot plane is the plane of symmetry, which should separate the cells into 2 equal half parts. The yellow vector " $\mathrm{V}_{\text {sym }}$ " is the vector for the plane of symmetry. The right part is the modiolar view of the cell. This panel simply visualizes the goodness of the separation. (B) The view at the plane of symmetry. The blue line represents for the contact of $\mathrm{IHC}$ to the pillar cell. The orange arrow is the central axis " $\mathrm{V}_{\mathrm{z}}$ ". The thick green dashed line represents for the largest cutting section. The central purple dot is the center of mass of the largest section. (C) Three orthogonal axes of $\mathrm{IHCs}$ are $\mathrm{V}_{\mathrm{z}}, \mathrm{V}_{\text {sym }}$ and $\mathrm{V}_{\mathrm{mp}}$. The transferred coordinate $\mathrm{r}, \mathrm{H}$, and $\theta$ are represented by their symbols in this figure. The right part represents the convention of polar charts used in figure III.9. 
Figure III.9B demonstrates the spatial distribution of the intensity of stained ribbon. The $F_{\text {ribbon }} / F_{\text {nearby }}$ had stronger intensities in modiolar parts, which might mean that the ribbon size is larger in the modiolar surface of IHC. This result is also consistent with previous study which estimated the size of the synaptic ribbons by measuring the intensity of labeled ribbons in immunostaing experiments (Liberman et al., 2011). Despite the modiolar part displays stronger intensities of stained ribbons, there were still quite some numbers of stained ribbons with weak and middle intensities.

Figure III.9A demonstrates the spatial distributions of $\mathrm{Ca}^{2+}$ microdomain intensity. The intensity was stronger in modiolar part than in pillar part when examining the $\Delta \mathrm{F}_{\text {avg }}$ (Figure III.9A $A_{7}$. In contrast, the $R_{a v g}$ did not exhibit a significant spatial preference in the modiolar-pillar direction (Figure III.9A $\mathrm{A}_{3}$. However, in figure III.9A 2 , the distribution in modiolar part shows comparably obvious more microdomains in strong intensities in than those in the pillar part. Therefore, I tried to judge whether the variation of distribution was different in modiolar-pillar direction. The Kruska-Wallis test was therefore applied to test the differences. However the result still did not report significant differences either. Because the intensities of resting $\mathrm{Ca}^{2+}$ indicator fluorescence $\left(\mathrm{F}_{0, \mathrm{Ca}^{2+}}\right.$ in figure III.7A) in the place where the $\mathrm{Ca}^{2+}$ microdomain appeared were linearly positive to the intensities $\left(F_{\text {ribbon }}\right)$ of the labeled ribbons (Figure III.7A), and the size of synaptic ribbons $\left(\mathrm{F}_{\text {ribbon }} / \mathrm{F}_{\text {nearby }}\right)$ were larger in modiolar part as well, the statistical difference in III.9A $\mathrm{A}_{7}$ might attribute to the stronger resting fluorescence of $\mathrm{Ca}^{2+}$ indicator in the synapses of the modiolar part thereby resulting more fluorescence when the $\mathrm{Ca}^{2+}$ microdomain was evoked.

The spatial distribution of voltage-activation properties, $\mathrm{V}_{0.5}$ and $\mathrm{k}$, are demonstrated in figure III.9C and D. $V_{0.5}$ was shown to be statistically more negative in pillar part than in modiolar part. This result might imply stronger voltage sensitivity of $\mathrm{Ca}_{v} 1.3$ in the pillar part 
which is possible to result in lower acoustic threshold when the connected SGN is stimulated by sound levels. As well, even though the statistical difference was observed, there were still a considerable number of the $\mathrm{Ca}^{2+}$ microdomains with moderate $\mathrm{V}_{0.5}$ both in modiolar and pillar parts. Combining the result of figure $I I I .9 A_{3}, B_{3}$, and $C_{3}$, although the spatial preferences of ribbon size and voltage-activation of $\mathrm{Ca}^{2+}$ microdomains were observed, there is no clear division in $\mathrm{IHC}$ to spatially identify different groups of these synaptic properties according to their intensities. This observation is different from what were observed of previous studies where an obvious division can be found to spatially separate different intensities of the stained ribbons into two groups (Liberman et al., 2011; Lin et al., 2011). Spatial comparison of the rate constant $k$ was examined (Figure $\left.I I I .9 D_{3}\right)$, however there was no spatial preference exhibiting. In figure III.9D ${ }_{1}$ and $D_{2}$ the $k$ of modiolar part also displays similar distribution with pillar parts.

The spatial preference in the direction of tonotopical axis was also investigated in this study, which has not yet been examined before. In figure III.9A $\mathrm{A}_{4}, \mathrm{~B}_{4}, \mathrm{C}_{4}$ and $\mathrm{D}_{4}$, I compared the distribution in tonotopical apical and basal part of $\mathrm{Ca}^{2+}$ microdomain intensity, stained ribbon intensity, $\bigvee_{0.5}$ and $k$ respectively. However, no any statistical differences were observed, and the distributions of these properties in both parts look similar too. 

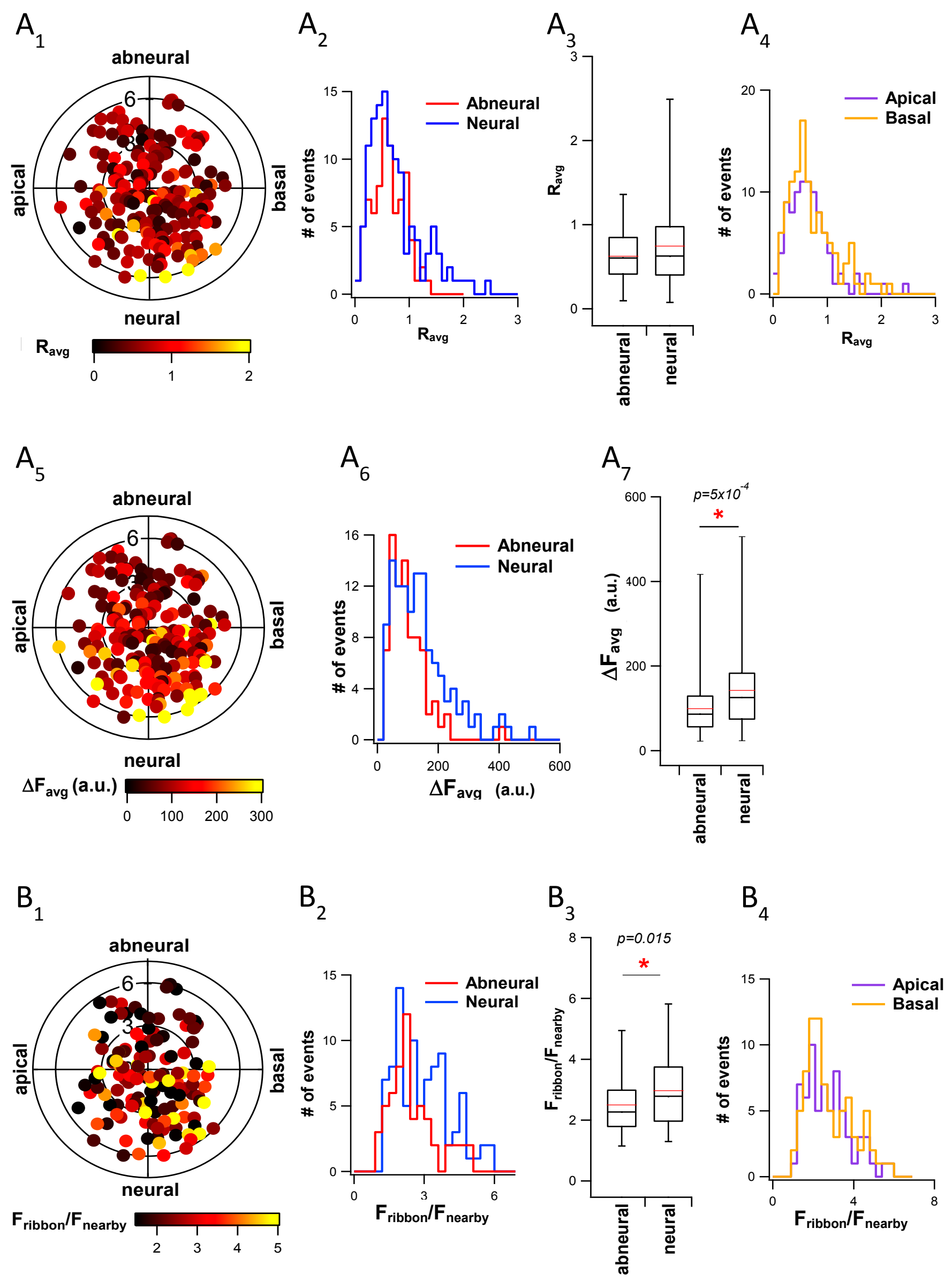

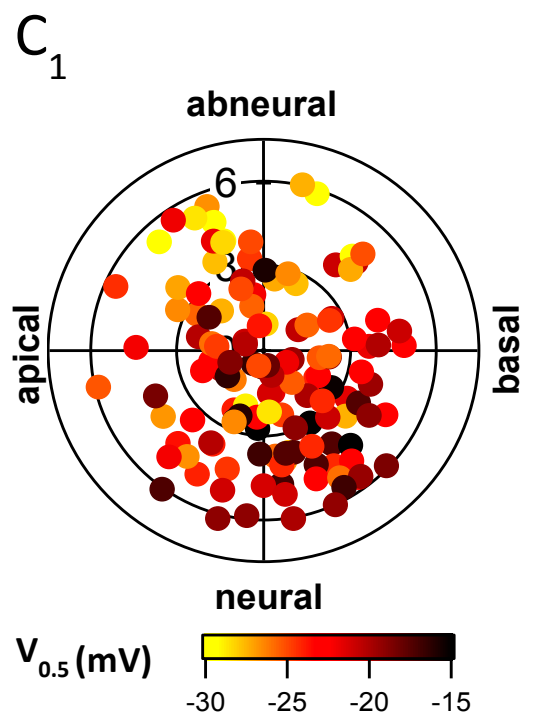

$D_{1}$

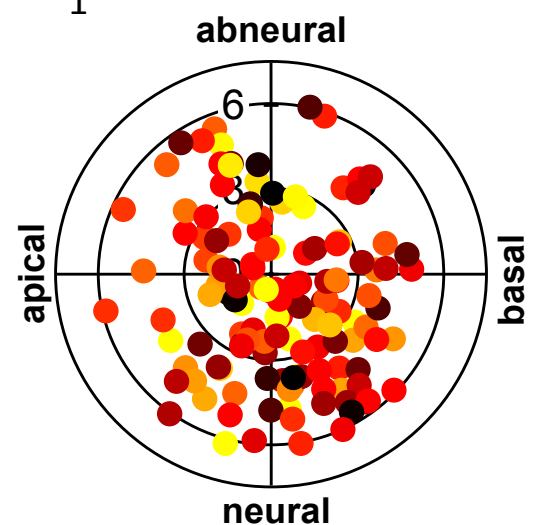

k (mV)
$C_{2}$

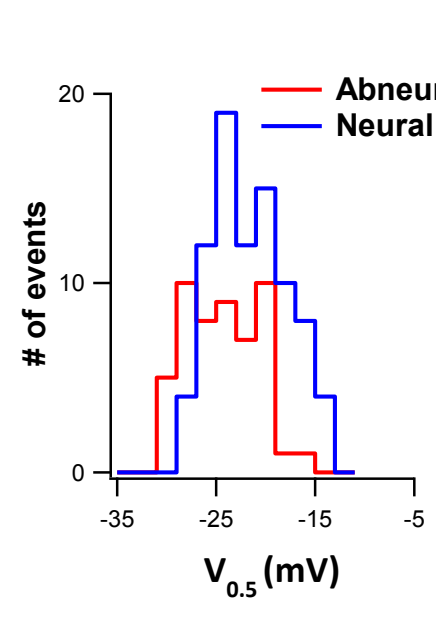

$D_{2}$

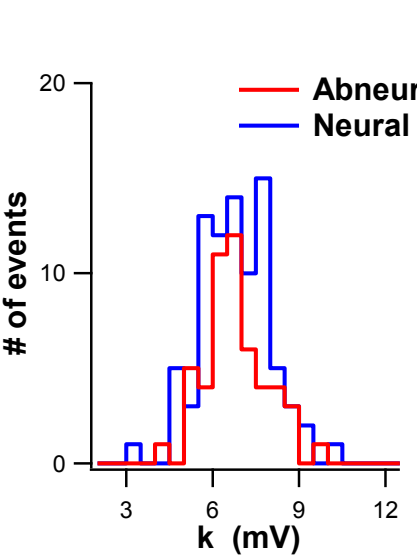

$\mathrm{C}_{3}$

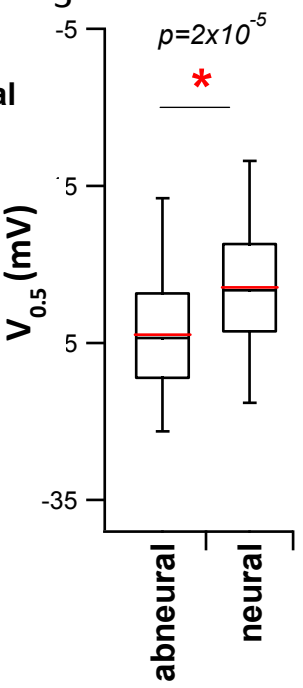

$D_{3}$

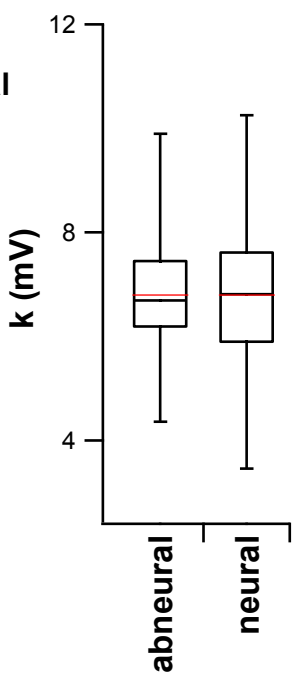

$\mathrm{C}_{4}$

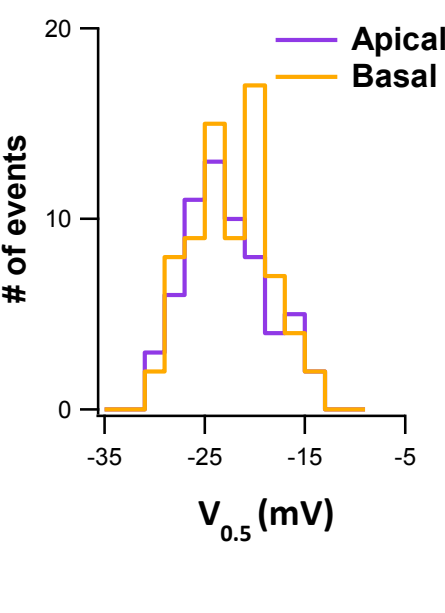

$\mathrm{D}_{4}$

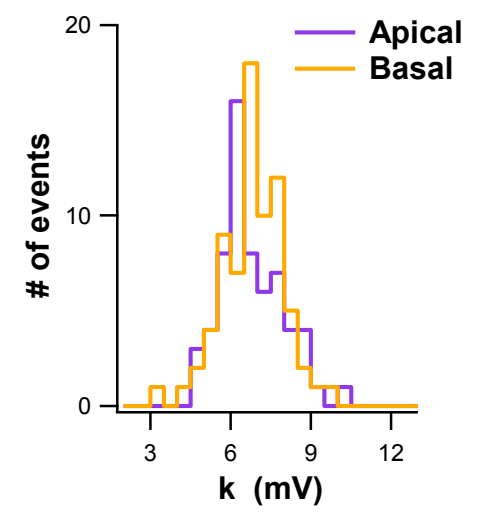




\section{Figure III.9}

\section{Polar distribution of properties of $\mathrm{Ca}^{2+}$ microdomains and ribbons}

(A) illustrates the polar distribution of the $\mathrm{Ca}^{2+}$ microdomain intensities. $\mathrm{A}_{1}$ to $\mathrm{A}_{4}$ list different distributions of normalized intensity $R_{\text {avg }} . A_{5}$ to $A_{7}$ list those of measured intensity $\Delta F_{\text {avg. }} . A_{1}$ an $A_{5}$ display the overview of the spatial distribution of $\mathrm{Ca}^{2+}$ microdomain intensity in polar charts. $A_{2}$ and $A_{6}$ display the distribution in both modiolar and pillar parts. $A_{3}$ and $A_{7}$ compare their statistics. Notice that the statistical difference only exists in $A_{7}$. $A_{4}$ display the distribution in both tonotopical apical and basal part. There is no statistical difference for the $\mathrm{Ca}^{2+}$ microdomain intensity tonotopically so the statistical comparison is not shown here. From (B) to (D) illustrate the polar properties of the intensities of labeled ribbons $\left(F_{\text {ribbon }} / F_{\text {nearby }}\right), V_{0.5}$, and $k$ respectively. $B_{1}, C_{1}$, and $D_{1}$ display the overview of spatial distribution these properties. $B_{2}, C_{2}$ and $D_{2}$ display the distribution of them in both modiolar parts and pillar parts. $B_{3}, C_{3}$, and $D_{3}$ compare the difference of these properties from modiolar part to pillar part. $B_{4}, C_{4}$ and $D_{4}$ display the distribution of these properties in tonotopical axis. The box plot in $A_{3}, A_{7}, B_{3}, C_{3}$ and $D_{3}$ represent for $0,25 \%, 50 \%, 75 \%$ and $100 \%$ of the distribution in $A_{2}, A_{6}, B_{2}, C_{2}$, and $D_{2}$ respectively. The red lines in these box plots represent for the mean of these distributions. Notice that the statistical differences exist in $\mathrm{B}_{3}$ and $\mathrm{C}_{3}$, and the statistical difference were confirmed by nonparametric 2 tail Wilcoxin-Rank test under the confidence level $P_{\alpha}<0.05$. There is also no statistical difference for all the properties tonotopically in $\mathrm{B}_{4}, \mathrm{C}_{4}$, and $\mathrm{D}_{4}$.

\section{Longitudinal properties of microdomains and ribbons do not show differences}

Previous work had identified anatomical differences among the postsynaptic boutons of spiral ganglion neurons which innervate the IHC at different positions (Francis et al., 2004). Simple and smaller boutons were located basally whereas larger boutons with folded structure resided more apically. Except for that, little is known about the characteristics of synaptic ribbons, $\mathrm{Ca}^{2+}$ microdomains and the physiological properties of postsynaptic SGNs along the long axis a hair cells. Here all four properties, maximum intensity $\left(R_{\text {avg }}\right)$, ribbon fluorescence, $\mathrm{V}_{0.5}$ and $\mathrm{k}$ were inspected as a function of position on the long axis of the IHC. Figurelll.10A to $\mathrm{D}$ displays the respective relationships: none of the four properties showed a significant correlation with the long axis. 

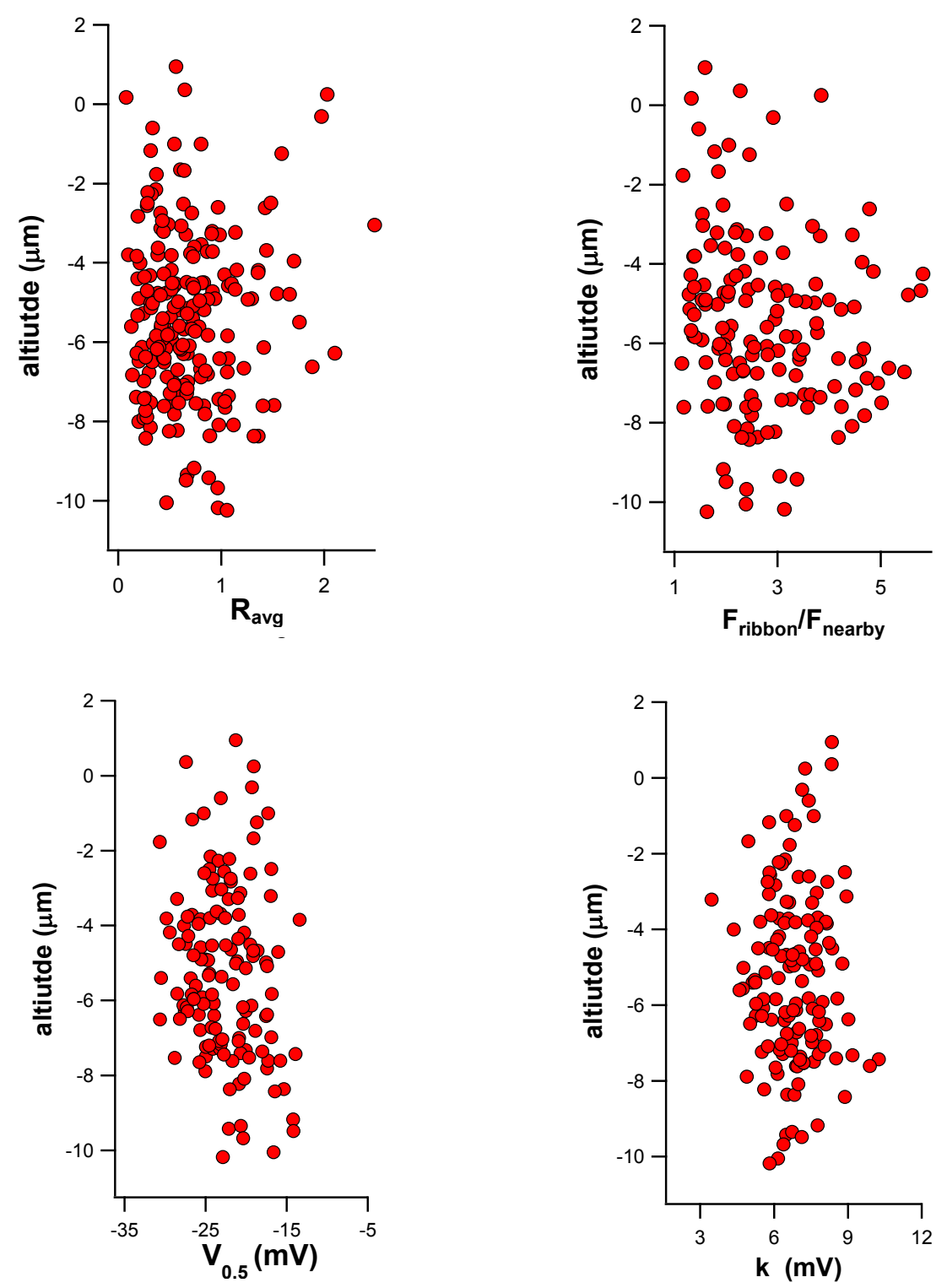

Figure III.10

Altitudinal distribution of properties of $\mathrm{Ca}^{2+}$ microdomains and ribbons

(A),(B),(C),(D) list the relationship between altitude and relative properties, $R_{\text {avg }}, F_{\text {ribbon }} / F_{\text {nearby }}, V_{0.5}, k$ respectively. According to the cylindrical definition of cells, the 0 in altitude axis was set to be the center of mass of the largest section along longitudinal axis $\mathrm{V}_{\mathrm{z}}$ (Figure III.8B), and negative value indicated the position more basal than largest section. In this figure all four variables do not show a significant positive or negative relationship with the altitude. 


\section{Discussion}

\section{Heterogeneity of presynaptic $\mathrm{Ca}^{2+}$ signals within and across IHCs}

In this study, I demonstrated diversity of $\mathrm{Ca}^{2+}$ microdomains in numerous properties. Comparing to the previous study (Frank et al., 2009), which displayed heterogeneous maximal intensities and voltage-dependencies of $\mathrm{Ca}^{2+}$ microdomains across several cells and indicated heterogeneity also within individual cells, my work extended this notion by observing all $\mathrm{Ca}^{2+}$ microdomain of an individual cell. Indeed, using a different imaging approach and focusing on "within cell" comparisons I found marked heterogeneity of maximal intensities and voltage-dependencies of $\mathrm{Ca}^{2+}$ microdomains. The C.V. of the $\Delta \mathrm{F}$ distribution among all spots was comparable to the previous study $(0.70$, figure III.4A, as compared to 0.66 (Frank et al., 2009)). However, the $\Delta \mathrm{F}$ variance differed between the individual IHCs. The grand average of the C.V. of all cells was 0.54 , whereby the largest and smallest C.V. were 1.00 and 0.14 , respectively. Different extents of heterogeneity among the cells may imply that, in addition to the most likely comparable external regulation, cells may also use cell-autonomous mechanisms to determine the heterogeneity among their synapses.

In addition to the $\Delta \mathrm{F}$, the ratio of $\Delta \mathrm{F}$ to rest fluorescence, $\mathrm{R}_{\mathrm{avg}}$, of evoked $\mathrm{Ca}^{2+}$ microdomains needed to be assessed, because background fluorescence in resting conditions differed among the active zones. This variable background might result from slight deviations of dye concentration, incomplete loading of the cell or inhomogeneous dye concentration within the cytosol, or different contributions of out-of-focus fluorescence. The $\mathrm{Ca}^{2+}$ indicator Fluo-3 was found to accumulate at synaptic ribbons (Issa and Hudspeth, 1996). The $\mathrm{Ca}^{2+}$ indicator used in these experiments (Fluo-8FF) is a derivate of Fluo-3, so it appears possible that Fluo-8FF also attached to the synaptic ribbon. Indeed, the resting $\mathrm{Ca}^{2+}$ indicator's fluorescence at the 
synapse was positively correlated to the fluorescence of the corresponding peptide-stained ribbons (Fig III.7A), but not to the fluorescence of the nearby regions which surrounded the corresponding ribbons in the colour channel of the fluorescent peptide $\left(F_{\text {nearby, }}\right.$ fig III.7B). Hence, $\mathrm{R}_{\text {avg }}$ instead of $\Delta \mathrm{F}_{\text {avg }}$ was preferred for assessing the intensity of $\mathrm{Ca}^{2+}$ microdomains. Although the global variability of $R_{a v g}$ is smaller than $\Delta F_{a v g}$, the distribution of $R_{a v g}$ still displayed high diversity with a C.V. of approximately 0.6. Again C.V.s varied among the cells (mean: 0.47 , largest: 0.81 smallest: 0.15 ), but not as much as for $\Delta F_{\text {avg. }}$ In summary, spinning disk confocal $\mathrm{Ca}^{2+}$ imaging corroborates the previous finding of a stark heterogeneity of the intensity of presynaptic $\mathrm{Ca}^{2+}$ microdomains.

Several plausible mechanisms can be considered to contribute the heterogeneous intensities of $\mathrm{Ca}^{2+}$ microdomains. First of all, variable numbers of $\mathrm{Ca}_{\mathrm{v}} 1.3$ at individual ribbon synapses can result in different intensities of $\mathrm{Ca}^{2+}$ influx. Measuring the ion current on the small presynaptic membrane patch can obtain an estimate of the channel number. However, the active zone is usually covered by postsynaptic boutons. Moreover, without the information of the position of synaptic ribbons even makes it more difficult to precisely patch-clamp the $\mathrm{Ca}^{2+}$ channel cluster at the active zone. Instead, in the ruptured whole-cell patch-clamp condition, the positions of synapses can be confirmed by the ribbon staining peptide. Subsequent measurement of the reduction of whole-cell $\mathrm{Ca}^{2+}$ current while selectively blocking the $\mathrm{Ca}^{2+}$ influx at a single microdomain is probably practical to estimate the numbers of channels on site.

\section{Mechanisms for the heterogeneity of voltage-dependent activation of $\mathrm{Ca}^{2+}$ microdomains}

Besides the different maximal intensities of $\mathrm{Ca}^{2+}$ microdomains, I also found the voltage dependence of activation to differ among the $\mathrm{Ca}^{2+}$ microdomains (Fig. III.5). Interestingly, 
the mean $\mathrm{V}_{0.5}$ of the evoked $\mathrm{Ca}^{2+}$ microdomains in fig. III.5 is around $6 \mathrm{mV}$ more positive than that of the whole cell $\mathrm{Ca}^{2+}$ current (Fig III.5C). The more positive $\mathrm{V}_{0.5}$ might be due to the high $\left[\mathrm{Cs}^{+}\right]$inside the patch pipette. $\mathrm{Cs}^{+}$has been shown to permeate L-type $\mathrm{Ca}^{2+}$ channels in cardiomyocytes (Hess et al., 1986). In my experiments, the ratio of internal to external $\left[\mathrm{Cs}^{+}\right]$is larger than 100 , which can result in a negative shift of the current's reversal potential. In addition, the slow-activated $\mathrm{Ca}^{2+}$ dependence $\mathrm{K}+$ channels (SK), which cannot be blocked thoroughly by $\mathrm{Cs}^{+}$and tetraethlyammonium (TEA), might also be activated in the later part of the ramp-depolarization protocol because of the $\mathrm{Ca}^{2+}$ influx thereby increasing $\mathrm{K}^{+}$conductance in the whole cell ion current. Hence, the whole-cell currents measured in the patch-clamp experiments might not be carried by $\mathrm{Ca}^{2+}$ alone, but it is also possible that an additional $\mathrm{Cs}^{+}$or $\mathrm{K}^{+}$current were measured. As a result, the whole-cell $\mathrm{V}_{0.5}$ could be negatively shifted because of the negative reversal potential of $\mathrm{Cs}^{+}$, while the $\mathrm{V}_{0.5}$ measured by $\mathrm{Ca}^{2+}$ imaging is not affected by $\mathrm{K}^{+}$and $\mathrm{Cs}^{+}$currents In this point of view, the voltage-dependent activation of $\mathrm{Ca}^{2+}$ channels can be better estimated by measuring the evoked fluorescence of $\mathrm{Ca}^{2+}$ microdomain than by the whole-cell ion current.

In addition to the reason described above, the estimation of $V_{0.5}$ could also have deviated owing to the error in determining the reversal potential of $\mathrm{Ca}^{2+}$ for the estimation of the voltage dependence of the activation of $R_{\text {avg. }}$. The reversal potential should have been determined empirically by linear extrapolation. The fluorescence/current traces in the range from 0 to $15 \mathrm{mV}$ were usually linear. We estimated the reversal potential from the crossing of the extrapolated line with the resting signal. However, the ramp depolarization protocol used here only went up to potentials of maximally $+13 \mathrm{mV}$, which is not sufficiently positive to estimate the reversal potential through the extrapolation. Therefore in this experiment the reversal potential was estimated in the step IV curves, which were recorded in the beginning of the whole-cell patch-clamp recording. The difference of the resulting reversal potentials 
between cells was not larger than $5 \mathrm{mV}$. In addition, the noisy background fluorescence in resting (stimulation-free) condition can cause uncertainty in the estimation of the true fluorescence change. Both reasons can also result in the deviation of the estimation of $V_{0.5}$ from the $\mathrm{Ca}^{2+}$ fluorescence. Despite the difference of $\mathrm{V}_{0.5}$ between ion current and $\mathrm{Ca}^{2+}$ fluorescence, since the reversal potential was fixed in the range between $+37 \mathrm{mV}$ to $+42 \mathrm{mV}$ among cells, analyzing the variability of $\mathrm{V}_{0.5}$ and $\mathrm{k}$ is still practical in this study.

This difference in $V_{0.5}$ might originate from different molecular mechanisms regulating the properties of $\mathrm{Ca}_{v} 1.3$. As mentioned in the introduction, numerous alternative splice variants of $\mathrm{Ca}_{\mathrm{v}} 1.3$ were identified in the nervous system as well as the organ of Corti (Tan et al., 2011b). In HEK cells, most of these splice variants have been shown to affect the activation and inactivation of $\mathrm{Ca}_{v} 1.3$. For example, one variant of $\mathrm{Ca}_{v} 1.3$ with a deletion of the IQ domain in the C-terminal region has been observed in OHCs, and suggested to be responsible for the reduced amount of CDI (Shen et al., 2006). Hence, concomitant expression of several splice variants within a single $\mathrm{IHC}$ and their specific trafficking/stabilization at the various active zones might be a mechanism to explain the heterogeneity of channel activation.

The characteristics of voltage-dependent activation of one single splice variant should be conserved, which means a stable coefficient of $\mathrm{V}_{0.5}$ and $\mathrm{k}$. So if a $\mathrm{Ca}^{2+}$ microdomain uses a single splice variant but splice variants differ between the microdomains in an IHC, observation of a multi-Gaussian distribution of the $\mathrm{V}_{0.5}$ and $\mathrm{k}$ could be expected, whereby the different modes of the distribution may resemble the characteristic $V_{0.5}$ values of the $C a_{v} 1.3$ splice variants. However, a single normal distribution was observed (Fig. III.5D,E). Therefore, I favor the view that the $\mathrm{Ca}^{2+}$ channel cluster of each ribbon synapse is composed of a combination of several splice variants of the $\alpha_{1 D}$ subunit. 
Those $\mathrm{Ca}^{2+}$ microdomains with stronger intensities tend to manifest moderate $\mathrm{V}_{0.5}$ and $\mathrm{k}$. Weaker $\mathrm{Ca}^{2+}$ microdomains exhibit more extreme $\mathrm{V}_{0.5}$ and $\mathrm{k}$, either more negative/faster or positive/slower (Fig. III.5F,G). The majority of the $\mathrm{Ca}^{2+}$ microdomains presented with moderate $V_{0.5}$ and $k$, which might mean that there is one main splice variant of the $\alpha_{1 D}$ subunit with moderate $\mathrm{V}_{0.5}$ and $\mathrm{k}$ expressed in IHCs. Assuming that the probability of expressing a specific isoform of $\alpha_{1 \mathrm{D}}$ in a $\mathrm{Ca}^{2+}$ channel cluster is independent to other isoforms or channels, the expression of this main isoform would dominate the characteristics of the voltage-dependent activation of every $\mathrm{Ca}^{2+}$ channel cluster and thereby reduce the variability of the $\mathrm{V}_{0.5}$ and $\mathrm{k}$ of the evoked $\mathrm{Ca}^{2+}$ microdomains. It is more difficult than to explain why the weaker $\mathrm{Ca}^{2+}$ microdomain tend to have extreme $\mathrm{V}_{0.5}$ and $\mathrm{k}$ in figure III.5F,G.

On the contrary, the other possible mechanism could be that there are two major spice variants of which one exhibits extremely negative $V_{0.5}$ and small $k$ and the other one is opposite. The composition of a single microdomain could thereby be interpreted by the binomial distribution

$$
f(x)=\left(\begin{array}{l}
n \\
x
\end{array}\right) p^{x}(1-p)^{n-x}
$$

Here, $\mathrm{n}$ represents for the total channel number of $\mathrm{a} \mathrm{Ca}^{2+}$ channel cluster, which could be estimated by the intensity of $\mathrm{Ca}^{2+}$ microdomain. $\mathrm{x}$ represents the number of one specific splice variant of the $n$ channels thereby the number of the other variant is $n-x$. The parameter $p$ should be interpreted as expression probability of one variant. If the expression probability of both variants is the same, the value $p$ would be $0.5 . f(x)$ thereby represents for the probability to observe a $\mathrm{Ca}^{2+}$ channel cluster containing $\mathrm{x}$ number of one isoform from the cluster containing $\mathrm{n}$ channels. In this distribution, the weaker microdomains which are supposed to have fewer channels (small $n$ ) would exhibit a broad distribution where the microdomains with extreme $\mathrm{V}_{0.5}$ have high chances to be manifested. In contrast, a stronger 
$\mathrm{Ca}^{2+}$ microdomain which has large $\mathrm{n}$ would present a narrow distribution whereby fewer chances to observe a cluster nearly composing of one specific variant. Therefore, the binominal distribution is consistent with the finding that the $\mathrm{Ca}^{2+}$ microdomain with extreme $\mathrm{V}_{0.5}$ and $\mathrm{k}$ has are associated with low intensity. To accomplish the observation of figure III.5F,G, the expression of both isoforms (p) should be nearly the same to comply with the symmetric pattern in figure III.5F,G.

What if the expression of one channel splice variant in a cluster is not independent from the expression of other splice variants? In figure III.9C, the spatial distribution of $\mathrm{V}_{0.5}$ displays a gradient which has more negative $V_{0.5}$ in pillar end and the value of the $V_{0.5}$ increase toward modiolar end. This observation indicates that the expression of specific isoforms of Cav1.3 $3_{\alpha 1 \mathrm{D}}$ might be under control of external mechanisms or be regulated by the polarity of cell. Both environmental and intracellular regulation of the expression of splice variants would influence and determine where and how one $\mathrm{Ca}^{2+}$ channel will be expressed and result in the variability of the characteristics of the ribbon synapse and its $\mathrm{Ca}^{2+}$ microdomain. The concentration gradient of growth factors has been suggested to regulate organ growth (Chisholm and Firtel, 2004; Schwank and Basler, 2010). This spatial gradient of channel activation characteristics could also be explained by the concentration gradient of some growth factors which exhibit the gradient along the modiolar-pillar axis of IHCs.

Additionally, there is still no detailed investigation about the single-channel properties of different splice variants of the $\alpha 1 \mathrm{D}$ subunit. Whether the opening probability or single channel conductance is different also determine the intensity of $\mathrm{Ca}^{2+}$ influx and thereby could result in different intensities of $\mathrm{Ca}^{2+}$ microdomains. Therefore, detailed investigations of the diversity of $\mathrm{Ca}_{v} 1.3$ are necessary to understand the mechanisms of $\mathrm{Ca}^{2+}$ microdomain heterogeneity. 
Finally, the difference of $\mathrm{V}_{0.5}$ could explain the wide dynamic range of the sound-intensity encoding of the auditory system. A sound with an amplitude of around $100 \mathrm{~dB}$ can increase the receptor potential of IHCs to approximately $20 \mathrm{mV}$ (Dallos, 1985, 1986). The $4 \mathrm{mV}$ differences found for the $\mathrm{V}_{0.5}$ values in $\mathrm{Ca}^{2+}$ microdomains from different synapses are sufficiently high to endow the synapses with different operation ranges to collectively encode wide ranges of sound amplitude. Therefore, different synapses might utilize different operational ranges of the voltage activation of their presynaptic $\mathrm{Ca}^{2+}$ influx to encode different sound intensity ranges in the postsynaptic SGNs.

\section{The sizes of synaptic ribbons correlate with the intensities of $\mathrm{Ca}^{2+}$ microdomains}

The size of the synaptic ribbon dominates the function of the ribbon synapses. A big ribbon can hold more readily releasable vesicles (Khimich et al., 2005; Graydon et al., 2011), and probably more $\mathrm{Ca}^{2+}$ channels which was demonstrated by measurements of $\mathrm{Ca}^{2+}$ microdomains (Fig.III.7) and fluorescent-peptide labeling of the ribbons in this study. While I cannot provide a direct investigation of the relationship between the $\mathrm{Ca}^{2+}$ channel number and the RRP pool size in this study, it is very likely that the output of the synapse is governed by the number of functional release sites ("slots") composed of vesicular release sites and few nearby $\mathrm{Ca}^{2+}$ channels (Brandt et al., 2005; Goutman and Glowatzki, 2007; Graydon et al., 2011). Therefore, a positive correlation of the $\mathrm{Ca}^{2+}$ microdomain with the ribbon size likely also predicts more exocytosis at the larger ribbon synapses. I used the fluorescent CtBP2-binding peptide (Zenisek et al., 2003; Francis et al., 2011) to infer ribbon size. This seems valid because the number of RIBEYE molecules available for binding the peptide is expected to increase with size of the ribbon. Our results display a positive correlation of the 
intensity of ribbon staining to the fluorescence intensity of evoked $\mathrm{Ca}^{2+}$ microdomains at that synapse with $\mathrm{P}_{\mathrm{r}}=0.55$ (Fig.III.7D). This result suggests a larger ribbon could cluster more $\mathrm{Ca}^{2+}$ channels. Although this positive correlation is basically exhibited in most cells, the extent differed of the correlation between the cells. Potential reasons for these differences include i) the possibility that the some of the $\mathrm{Ca}^{2+}$ microdomain may have resulted from $\mathrm{Ca}^{2+}$ influx at neighboring ribbon-type active zones, of which only one was included into the intensity analysis, ii) that the fluorescent peptide binding did not always faithfully predict the active zones area or number of $\mathrm{Ca}^{2+}$ channels and iii) a contribution of mechanisms such as $\mathrm{Ca}^{2+}$ induced $\mathrm{Ca}^{2+}$ release to the $\mathrm{Ca}^{2+}$ microdomain (Frank et al., 2009).

Nevertheless, our current result does not provide information on how release of synaptic vesicles relates to the strength of $\mathrm{Ca}^{2+}$ microdomains. Visualizing synaptic vesicle numbers is difficult in the normal light microscopy where $\mathrm{Ca}^{2+}$ imaging and patch-clamp recording are performed. Applying electron microscopy subsequent to the $\mathrm{Ca}^{2+}$ imaging and the patch-clamp recording might be a possible solution to compare the physiological features with the corresponding presynaptic ultrastructure. In addition to the RRP, the application of the $\mathrm{Ca}^{2+}$ chelators EGTA or BAPTA were also proven to affect the rate of slow exocytosis in the measurement of cell membrane capacitance changes (Moser and Beutner, 2000; Goutman and Glowatzki, 2007), which implicated the $\mathrm{Ca}^{2+}$ also play some roles to influence the other vesicle pools or the sustained vesicle supplying in the ribbon synapses. The positive correlation shown in figure III.7 in this viewpoint might be able to suppose robust vesicle recruitment at large ribbons due to their stronger $\mathrm{Ca}^{2+}$ microdomains.

\section{Properties of $\mathrm{Ca}^{2+}$ microdomains exhibit spatial preference within IHCs}

A spatial preference of the SGNs with different functional properties has been revealed for the cat cochlea (Liberman, 1982; Merchan-Perez and Liberman, 1996) related to a spatial 
segregation of morphological synapse features: SGNs with high SR are more likely to innervate the pillar (neural) surfaces of IHCs, and to have larger diameters of the dendritic neurites but smaller synaptic ribbons (Merchan-Perez and Liberman, 1996). In agreement with the notion of smaller ribbons on the pillar side, immunostainings of IHC synaptic ribbons displayed lower fluorescence intensities on the pillar side of IHCs than on the modiolar side (Liberman et al., 2011).

In my experiments, I estimated the size of the synaptic ribbons by analyzing the fluorescence of peptide-labeled ribbons and reconstructed the shapes of living IHCs. Owing to my experimental requirement, I generated a definition based on the morphology of the cell to elucidate the orientation of the tested IHCs (Fig.III.8). The definition used in previous studies establishes the orientation of an IHC based on the circumferential landmarks (Liberman, 1982; Merchan-Perez and Liberman, 1996; Liberman et al., 2011). However, because the shape and position of each hair cell could be changed cell by cell and sample by sample, the resulting orientation of every IHC could be different from each other. A gentle movement of the cell can cause the deviation of the position determination from it should have been. How to deal with this error seemed not be clear described in those previous studies, however, in my study they were considered cell-by-cell.

In my analysis, stronger intensities of stained ribbons and more depolarized $\mathrm{V}_{0.5}$ of the $\mathrm{Ca}^{2+}$ microdomains were found on the modiolar surfaces (fig III.9B,C). While stronger $\mathrm{Ca}^{2+}$ microdomains have been shown at the modiolar side in a previous study (Meyer et al., 2009), I did not find a significant spatial gradient in my experiments but there was a corresponding trend. As described in the result, the distribution of the $\mathrm{Ca}^{2+}$ microdomain intensity on the modiolar side displays more events with large intensity than on the pillar side (Fig.III.9A 2 ). The stronger intensity of stained-ribbons in the modiolar side (Fig.III.9B 3 ) and the positive correlation of the ribbon intensity with the $\mathrm{Ca}^{2+}$ microdomain intensity (Fig.III.7) indicate that the larger ribbons on the modiolar side are likely having stronger $\mathrm{Ca}^{2+}$ microdomain. 
In spite of the observed spatial preference of ribbon size and $V_{0.5}$, a clear separation like Liberman et al., 2011 were not seen in this study, for either synapse property: there are still quite some weak-intensity ribbons and low- $\mathrm{V}_{0.5} \mathrm{Ca}^{2+}$ microdomains at the modiolar side and vice versa.

\section{Presynaptic and postsynaptic mechanisms of diverse spontaneous rate in SGNs}

Spontaneous firing of SGN is likely driven by $\mathrm{Ca}^{2+}$ influx driven exocytosis of IHCs (Sewell, 1984; Robertson and Paki, 2002). However, as mentioned, those SGNs with high SR have been suggested to preferentially innervate the pillar side, which is in contradiction with the spatial distribution of the intensities of $\mathrm{Ca}^{2+}$ microdomains in my study as well as our previous study (Meyer et al., 2009) where the stronger $\mathrm{Ca}^{2+}$ microdomain preferred to appear in modiolar side and probably did not present a clear cut of spatial segregation of $\mathrm{Ca}^{2+}$ microdomain intensity (Fig.III.9A). Interestingly, my findings now revealed a more negative $\mathrm{V}_{0.5}$ of the activation of $\mathrm{Ca}^{2+}$ microdomains on the pillar side which probably results in higher $\mathrm{Ca}^{2+}$ influx at the same membrane potential and thereby seems able to attribute the lower threshold of voltage-dependent channel activation and resulting high SR to the presynaptic voltage sensitivities.

Liberman et al. tried to explain the heterogeneity of spontaneous activities as well as the acoustic threshold of SGNs by the observation of larger sizes of postsynaptic glutamate receptor clusters and of thicker calibers of the fibers of SGNs (Liberman, 1982; Liberman et al., 2011). They also observed that the degeneration of SGNs following noise trauma was mostly affecting the SGNs innervating the modiolar side of IHCs and might be the cause of the elevation of the acoustic threshold in auditory brainstem response (Lin et al., 2011). However, the small size of the SGNs' postsynaptic boutons and the close position from the IHC to the heminode of Ranvier are probably more important to determine the generation of 
action potentials (Hossain et al., 2005; Rutherford et al., 2012) than the size of nerve fibers. Furthermore, spontaneous EPSPs were shown to be easily capable to evoke action potentials, which also indicates a low threshold and high accessibility for action potentials (Siegel, 1992). Similar results were also shown in patch-clamp recordings at the dendritic boutons of SGNs with slow spontaneous firing rates (Rutherford et al., 2012).

Does the number of glutamate receptors determine the spontaneous rates? Given the same amount of presynaptic glutamate release, the bouton with fewer glutamate receptors is likely to fire slower because these receptors are saturated and cannot respond to more released glutamate. However, every synapse can certainly fire faster than the SR, which means more availability of receptors for neurotransmitters. On the other hand, given the same volume of synaptic cleft, the bouton with more glutamate receptors is likely to result in more ion influx than that with few receptors. However, Liberman et al. demonstrated stronger glutamate receptor staining with weaker ribbon staining. The latter likely translates into fewer synaptic vesicles released at this synapse, which might attenuate the AP firing rate.

Furthermore, the different sizes of stained glutamate receptor clusters might indicate differences in the volume or surface area of boutons. As mentioned before, the size of the bouton might influence the membrane charging speed which can determine the AP firing rate. Indeed, there are two different morphologies found in electron microscopy studies of SGN boutons (Francis et al., 2004). Those boutons with smaller volume and simple structure have been found to innervate the basal part of IHCs, and those with larger volume as well as folded structure innervate the apical parts. No comparison has been made between these two morphologies in the immunostaining of glutamate receptors, and more detailed studies regarding the function of this difference are still absent. Is the size of synaptic ribbon related to the size of the postsynaptic bouton? In this study, I found no differences in the intensities 
of $\mathrm{Ca}^{2+}$ microdomains and the stained ribbons along the altitudinal axis of the $\mathrm{IHC}$ (figllI.10A,B). The current result thereby cannot relate the size of the ribbon to that of the bouton.

In summary, my study indicates a role of different presynaptic voltage dependence of $\mathrm{Ca}^{2+}$ channel activation for determining the spontaneous firing of the postsynaptic SGN. Moreover, I observed a preference of larger ribbons to reside on the modiolar face of the IHC and a corresponding tendency of $\mathrm{Ca}^{2+}$ microdomain strength. Future experiments will need to directly assess postsynaptic firing in combination with measurements and manipulations of presynaptic properties to directly probe the relationship between presynaptic $\mathrm{Ca}^{2+}$ signaling and SGN function.

\section{Mechanism for the encoding of sound amplitude in IHCs}

My finding of a more hyperpolarized voltage-dependent activation of the presynaptic $\mathrm{Ca}^{2+}$ channels at the pillar side indicated that the acoustic threshold of the postsynaptic SGN may be lower. Indeed, manipulating the L-type $\mathrm{Ca}^{2+}$ channel by applying its blocker nimodipine or its agonist BayK8644 can result in changes of acoustic threshold and spontaneous rates in SGNs (Robertson and Paki, 2002) and forward masking paradigms also can cause a brief elevation of the acoustic threshold in single fiber recordings (Harris and Dallos, 1979; Spassova et al., 2004). These results all implicate the importance of the regulation of $\mathrm{Ca}^{2+}$-dependent exocytosis in the determination of acoustic thresholds. Furthermore, a linear relationship has been demonstrated between $\mathrm{V}_{0.5}$ and $\mathrm{k}$ in this study (Fig.III.5H). The $\mathrm{Ca}^{2+}$ microdomains with more negative $\mathrm{V}_{0.5}$ also exhibit smaller $\mathrm{k}$, which indicates that those microdomains with lower thresholds also are more sensitive to changes in membrane potential. 
Although the dynamic range of a SGN could be explained by its acoustic threshold, as well as the basilar membrane nonlinearities (for detail, see Sachs and Abbas, 1974), the variability of slope-factor of voltage-dependent activation of $\mathrm{Ca}^{2+}$ channels, $\mathrm{k}$, and the number of functional release sites seem to be the other mechanisms to regulate the sensitivity of the sound amplitude encoding in SGNs.

In summary, the voltage sensitivity of the presynaptic $\mathrm{Ca}^{2+}$ channel cluster might play an important role to determine the auditory threshold as well as the dynamic range of its connecting auditory nerve. 


\section{References}

Altier, C., Garcia-Caballero, A., Simms, B., You, H., Chen, L., Walcher, J., Tedford, H.W., Hermosilla, T., and Zamponi, G.W. (2010). The Cav[beta] subunit prevents RFP2-mediated ubiquitination and proteasomal degradation of L-type channels. Nature Neuroscience 14, 173-180.

Berridge, M.J., Bootman, M.D., and Roderick, H.L. (2003). Calcium signalling: dynamics, homeostasis and remodelling. Nature Reviews Molecular Cell Biology 4, 517-529.

Brandt, A., Khimich, D., and Moser, T. (2005). Few CaV1. 3 channels regulate the exocytosis of a synaptic vesicle at the hair cell ribbon synapse. J Neurosci 25, 11577.

Calin-Jageman, I., and Lee, A. (2008). Cav1 L-type Ca2+ channel signaling complexes in neurons. Journal of Neurochemistry 105, 573-583.

Cheatham, M.A. (2004). Cochlear function in Prestin knockout mice. The Journal of Physiology 560, 821-830.

Chisholm, R.L., and Firtel, R.A. (2004). Insights into morphogenesis from a simple developmental system. Nature Reviews Molecular Cell Biology 5, 531-541.

Cui, G., Meyer, A.C., Calin-Jageman, I., Neef, J., Haeseleer, F., Moser, T., and Lee, A. (2007). Ca2+-binding proteins tune Ca2+-feedback to Cav1. 3 channels in mouse auditory hair cells. The Journal of Physiology 585, 791-803.

Dallos, P. (1985). Response characteristics of mammalian cochlear hair cells. J. Neurosci. 5, 1591-1608.

Dallos, P. (1986). Neurobiology of cochlear inner and outer hair cells: intracellular recordings. Hearing Research 22, 185-198.

Dick, O., Hack, I., Altrock, W.D., Garner, C.C., Gundelfinger, E.D., and Brandstätter, J.H. (2001). Localization of the presynaptic cytomatrix protein Piccolo at ribbon and conventional synapses in the rat retina: Comparison with Bassoon. The Journal of Comparative Neurology 439, 224-234.

Tom Dieck, S., Altrock, W.D., Kessels, M.M., Qualmann, B., Regus, H., Brauner, D., Fejtová, A., Bracko, O., Gundelfinger, E.D., and Brandstätter, J.H. (2005). Molecular dissection of the photoreceptor ribbon synapse: physical interaction of Bassoon and RIBEYE is essential for the assembly of the ribbon complex. J. Cell Biol 168, 825-836.

Fettiplace, R. (2006). Active hair bundle movements in auditory hair cells. The Journal of Physiology 576, 2936. 
Fettiplace, R., and Hackney, C.M. (2006). The sensory and motor roles of auditory hair cells. Nat Rev Neurosci 7, 19-29.

Francis, A.A., Mehta, B., and Zenisek, D. (2011). Development of new peptide-based tools for studying synaptic ribbon function. J Neurophysiol 106, 1028-1037.

Francis, H.W., Rivas, A., Lehar, M., and Ryugo, D.K. (2004). Two types of afferent terminals innervate cochlear inner hair cells in C57BL/6J mice. Brain Research 1016, 182-194.

Frank, T., Khimich, D., Neef, A., and Moser, T. (2009). Mechanisms contributing to synaptic Ca2+ signals and their heterogeneity in hair cells. Proceedings of the National Academy of Sciences 106, 4483.

Frank, T., Rutherford, M.A., Strenzke, N., Neef, A., Pangršič, T., Khimich, D., Fejtova, A., Gundelfinger, E.D., Liberman, M.C., Harke, B., et al. (2010). Bassoon and the Synaptic Ribbon Organize Ca2+ Channels and Vesicles to Add Release Sites and Promote Refilling. Neuron 68, 724-738.

Gebhart, M., Juhasz-Vedres, G., Zuccotti, A., Brandt, N., Engel, J., Trockenbacher, A., Kaur, G., Obermair, G.J., Knipper, M., Koschak, A., et al. (2010). Modulation of Cav1.3 Ca2+ channel gating by Rab3 interacting molecule. Molecular and Cellular Neuroscience 44, 246-259.

Geisler, C.D. (1998). From Sound to Synapse (New York: Oxford University Press).

Glowatzki, E., and Fuchs, P.A. (2002). Transmitter release at the hair cell ribbon synapse. Nat. Neurosci 5, 147-154.

Goutman, J.D., and Glowatzki, E. (2007). Time course and calcium dependence of transmitter release at a single ribbon synapse. Proceedings of the National Academy of Sciences 104, 16341-16346.

Gräf, R., Rietdorf, J., and Zimmermann, T. (2005). Live Cell Spinning Disk Microscopy. In Microscopy Techniques, J. Rietdorf, ed. (Springer Berlin / Heidelberg), pp. 1311-1315.

Grant, L., Yi, E., and Glowatzki, E. (2010). Two Modes of Release Shape the Postsynaptic Response at the Inner Hair Cell Ribbon Synapse. J. Neurosci. 30, 4210-4220.

Graydon, C.W., Cho, S., Li, G.-L., Kachar, B., and Von Gersdorff, H. (2011). Sharp Ca2+ Nanodomains beneath the Ribbon Promote Highly Synchronous Multivesicular Release at Hair Cell Synapses. J. Neurosci. 31, 16637-16650.

Griesinger, C.B., Richards, C.D., and Ashmore, J.F. (2005). Fast vesicle replenishment allows indefatigable signalling at the first auditory synapse. Nature 435, 212-215.

Hackney, C.M., Mahendrasingam, S., Penn, A., and Fettiplace, R. (2005). The concentrations of calcium buffering proteins in mammalian cochlear hair cells. The Journal of Neuroscience 25, 7867. 
Harris, D.M., and Dallos, P. (1979). Forward masking of auditory nerve fiber responses. J. Neurophysiol. 42, 1083-1107.

Hess, P., Lansman, J.B., and Tsien, R.W. (1986). Calcium channel selectivity for divalent and monovalent cations. Voltage and concentration dependence of single channel current in ventricular heart cells. J Gen Physiol 88, 293-319.

Holderith, N., Lorincz, A., Katona, G., Rózsa, B., Kulik, A., Watanabe, M., and Nusser, Z. (2012). Release probability of hippocampal glutamatergic terminals scales with the size of the active zone. Nature Neuroscience 15, 988-997.

Hoppa, M.B., Lana, B., Margas, W., Dolphin, A.C., and Ryan, T.A. (2012). $\alpha 2 \delta$ expression sets presynaptic calcium channel abundance and release probability. Nature 486, 122-125.

Hossain, W.A., Antic, S.D., Yang, Y., Rasband, M.N., and Morest, D.K. (2005). Where is the spike generator of the cochlear nerve? Voltage-gated sodium channels in the mouse cochlea. The Journal of Neuroscience 25, 6857 .

Howard, J., and Hudspeth, A.J. (1988). Compliance of the hair bundle associated with gating of mechanoelectrical transduction channels in the bullfrog's saccular hair cell. Neuron 1, 189-199.

Hudspeth, A.J. (2008). Making an Effort to Listen: Mechanical Amplification in the Ear. Neuron 59, 530-545.

Issa, N., and Hudspeth, A. (1996). Characterization of fluo-3 labelling of dense bodies at the hair cell's presynaptic active zone. Journal of Neurocytology 25, 257-266.

Jenkins, M.A., Christel, C.J., Jiao, Y., Abiria, S., Kim, K.Y., Usachev, Y.M., Obermair, G.J., Colbran, R.J., and Lee, A. (2010). Ca2+-dependent facilitation of Cav1.3 Ca2+ channels by densin and Ca2+/calmodulin-dependent protein kinase II. J. Neurosci. 30, 5125-5135.

Juhasz-Vedres, G., Gebhart, M., Scharinger, A., Jangsangthong, W., Busquet, P., Poggiani, C., Sartori, S., Mangoni, M.E., Sinnegger-Brauns, M.J., Herzig, S., et al. (2011). Functional properties of a newly identified C-terminal splice variant of Cav1.3 L-type Ca2+ channels. Journal of Biological Chemistry.

Juusola, M., French, A.S., Uusitalo, R.O., and Weckström, M. (1996). Information processing by graded-potential transmission through tonically active synapses. Trends in Neurosciences 19, 292-297.

Khimich, D., Nouvian, R., Pujol, R., Tom Dieck, S., Egner, A., Gundelfinger, E.D., and Moser, T. (2005). Hair cell synaptic ribbons are essential for synchronous auditory signalling. Nature 434, 889-894.

Koschak, A. (2001). alpha 1D (Cav1.3) Subunits Can Form L-type Ca2+ Channels Activating at Negative Voltages. Journal of Biological Chemistry 276, 22100-22106. 
Koschak, A. (2010). Impact of gating modulation in Cav1.3 L-type calcium channels. Channels 4, 52-51.

Legan, P.K., Lukashkina, V.A., Goodyear, R.J., Kössl, M., Russell, I.J., and Richardson, G.P. (2000). A Targeted Deletion in $\alpha$-Tectorin Reveals that the Tectorial Membrane Is Required for the Gain and Timing of Cochlear Feedback. Neuron 28, 273-285.

Lenzi, D., Crum, J., Ellisman, M.H., and Roberts, W.M. (2002). Depolarization redistributes synaptic membrane and creates a gradient of vesicles on the synaptic body at a ribbon synapse. Neuron 36, 649-659.

Lenzi, D., Runyeon, J.W., Crum, J., Ellisman, M.H., and Roberts, W.M. (1999). Synaptic vesicle populations in saccular hair cells reconstructed by electron tomography. The Journal of Neuroscience 19, 119.

Liberman, L.D., Wang, H., and Liberman, M.C. (2011). Opposing Gradients of Ribbon Size and AMPA Receptor Expression Underlie Sensitivity Differences among Cochlear-Nerve/Hair-Cell Synapses. J. Neurosci. 31, 801808.

Liberman, M. (1982). Single-neuron labeling in the cat auditory nerve. Science 216, $1239-1241$.

Lin, H.W., Furman, A.C., Kujawa, S.G., and Liberman, M.C. (2011). Primary neural degeneration in the Guinea pig cochlea after reversible noise-induced threshold shift. J. Assoc. Res. Otolaryngol 12, 605-616.

Lipscombe, D., Pan, J., and Gray, A. (2002). Functional diversity in neuronal voltage-gated calcium channels by alternative splicing of Cava1; Molecular Neurobiology 26, 21-44.

Magupalli, V.G., Schwarz, K., Alpadi, K., Natarajan, S., Seigel, G.M., and Schmitz, F. (2008). Multiple RIBEYE-RIBEYE interactions create a dynamic scaffold for the formation of synaptic ribbons. J. Neurosci 28 , 7954-7967.

Matthews, G., and Fuchs, P. (2010). The diverse roles of ribbon synapses in sensory neurotransmission. Nat. Rev. Neurosci 11, 812-822.

Merchan-Perez, A., and Liberman, M.C. (1996). Ultrastructural differences among afferent synapses on cochlear hair cells: correlations with spontaneous discharge rate. J. Comp. Neurol 371, 208-221.

Meyer, A.C., Frank, T., Khimich, D., Hoch, G., Riedel, D., Chapochnikov, N.M., Yarin, Y.M., Harke, B., Hell, S.W., Egner, A., et al. (2009). Tuning of synapse number, structure and function in the cochlea. Nat Neurosci $12,444-453$.

Meyer, A.C., Neher, E., and Schneggenburger, R. (2001). Estimation of Quantal Size and Number of Functional Active Zones at the Calyx of Held Synapse by Nonstationary EPSC Variance Analysis. The Journal of Neuroscience 21, $7889-7900$.

Moser, T., and Beutner, D. (2000). Kinetics of exocytosis and endocytosis at the cochlear inner hair cell afferent 
synapse of the mouse. Proceedings of the National Academy of Sciences of the United States of America 97, 883.

Moser, T., Brandt, A., and Lysakowski, A. (2006a). Hair cell ribbon synapses. Cell Tissue Res 326, 347-359.

Moser, T., Neef, A., and Khimich, D. (2006b). Mechanisms underlying the temporal precision of sound coding at the inner hair cell ribbon synapse. The Journal of Physiology 576, 55-62.

Muresan, V., Lyass, A., and Schnapp, B.J. (1999). The Kinesin Motor KIF3A Is a Component of the Presynaptic Ribbon in Vertebrate Photoreceptors. J. Neurosci. 19, 1027-1037.

Naraghi, M., and Neher, E. (1997). Linearized buffered Ca2+ diffusion in microdomains and its implications for calculation of [Ca2+] at the mouth of a calcium channel. The Journal of Neuroscience 17, 6961.

Neef, J., Gehrt, A., Bulankina, A.V., Meyer, A.C., Riedel, D., Gregg, R.G., Strenzke, N., and Moser, T. (2009). The Ca2+ Channel Subunit beta2 Regulates Ca2+ Channel Abundance and Function in Inner Hair Cells and Is Required for Hearing. The Journal of Neuroscience 29, 10730.

Nouvian, R., Beutner, D., Parsons, T.D., and Moser, T. (2006). Structure and Function of the Hair Cell Ribbon Synapse. J Membrane Biol 209, 153-165.

Oliveria, S.F., Dell'Acqua, M.L., and Sather, W.A. (2007). AKAP79/150 Anchoring of Calcineurin Controls Neuronal L-Type Ca2+ Channel Activity and Nuclear Signaling. Neuron 55, 261-275.

Peterson, B.Z., DeMaria, C.D., Adelman, J.P., and Yue, D.T. (1999). Calmodulin is the Ca2+ sensor for Ca2+ -dependent inactivation of L-type calcium channels. Neuron 22, 549-558.

Platzer, J., Engel, J., Schrott-Fischer, A., Stephan, K., Bova, S., Chen, H., Zheng, H., and Striessnig, J. (2000). Congenital deafness and sinoatrial node dysfunction in mice lacking class D L-type Ca2+ channels. Cell 102, 89-97.

Roberts, W.M., Jacobs, R.A., and Hudspeth, A.J. (1990). Colocalization of ion channels involved in frequency selectivity and synaptic transmission at presynaptic active zones of hair cells. J. Neurosci 10, 3664-3684.

Robertson, D., and Paki, B. (2002). Role of L-Type Ca2+ Channels in Transmitter Release From Mammalian Inner Hair Cells. II. Single-Neuron Activity. Journal of Neurophysiology 87, 2734 -2740.

Robles, L., and Ruggero, M.A. (2001). Mechanics of the Mammalian Cochlea. Physiol Rev 81, 1305-1352.

Roux, I., Hosie, S., Johnson, S.L., Bahloul, A., Cayet, N., Nouaille, S., Kros, C.J., Petit, C., and Safieddine, S. (2009). Myosin VI is required for the proper maturation and function of inner hair cell ribbon synapses. Hum. Mol. Genet. 18, 4615-4628. 
Ruggero, M.A., Rich, N.C., Recio, A., Narayan, S.S., and Robles, L. (1997). Basilar-membrane responses to tones at the base of the chinchilla cochlea. The Journal of the Acoustical Society of America 101, 2151-2163.

Rutherford, M.A., Chapochnikov, N.M., and Moser, T. (2012). Spike Encoding of Neurotransmitter Release Timing by Spiral Ganglion Neurons of the Cochlea. J. Neurosci. 32, 4773-4789.

Sachs, M.B., and Abbas, P.J. (1974). Rate versus level functions for auditory-nerve fibers in cats: tone-burst stimuli. The Journal of the Acoustical Society of America 56, 1835-1847.

Schmitz, F., Königstorfer, A., and Südhof, T.C. (2000). RIBEYE, a component of synaptic ribbons: a protein's journey through evolution provides insight into synaptic ribbon function. Neuron 28, 857-872.

Schrauwen, I., Helfmann, S., Inagaki, A., Predoehl, F., Tabatabaiefar, M.A., Picher, M.M., Sommen, M., Seco, C.Z., Oostrik, J., Kremer, H., et al. (2012). A Mutation in CABP2, Expressed in Cochlear Hair Cells, Causes Autosomal-Recessive Hearing Impairment. Am. J. Hum. Genet.

Schwank, G., and Basler, K. (2010). Regulation of Organ Growth by Morphogen Gradients. Cold Spring Harb Perspect Biol 2,.

Schwarz, K., Natarajan, S., Kassas, N., Vitale, N., and Schmitz, F. (2011). The synaptic ribbon is a site of phosphatidic acid generation in ribbon synapses. J. Neurosci. 31, 15996-16011.

Sewell, W.F. (1984). The relation between the endocochlear potential and spontaneous activity in auditory nerve fibres of the cat. The Journal of Physiology 347, $685-696$.

Shen, Y., Yu, D., Hiel, H., Liao, P., Yue, D.T., Fuchs, P.A., and Soong, T.W. (2006). Alternative splicing of the $\mathrm{Ca}(\mathrm{v}) 1.3$ channel IQ domain, a molecular switch for Ca2+-dependent inactivation within auditory hair cells. J. Neurosci 26, 10690-10699.

Siegel, J.H. (1992). Spontaneous synaptic potentials from afferent terminals in the guinea pig cochlea. Hearing Research 59, 85-92.

Singh, A., Gebhart, M., Fritsch, R., Sinnegger-Brauns, M.J., Poggiani, C., Hoda, J.-C., Engel, J., Romanin, C., Striessnig, J., and Koschak, A. (2008). Modulation of Voltage- and Ca2+-dependent Gating of CaV1.3 L-type Calcium Channels by Alternative Splicing of a C-terminal Regulatory Domain. Journal of Biological Chemistry 283, 20733-20744.

Spassova, M.A., Avissar, M., Furman, A.C., Crumling, M.A., Saunders, J.C., and Parsons, T.D. (2004). Evidence that rapid vesicle replenishment of the synaptic ribbon mediates recovery from short-term adaptation at the hair cell afferent synapse. J. Assoc. Res. Otolaryngol 5, 376-390.

Sterling, P., and Matthews, G. (2005). Structure and function of ribbon synapses. Trends in Neurosciences 28, 
20-29.

Südhof, T.C. (2012). The Presynaptic Active Zone. Neuron 75, 11-25.

Tan, B.Z., Jiang, F., Tan, M.Y., Yu, D., Huang, H., Shen, Y., and Soong, T.W. (2011a). Functional characterization of alternative splicing in the C-terminus of L-type CaV1.3 channels. Journal of Biological Chemistry.

Tan, B.Z., Jiang, F., Tan, M.Y., Yu, D., Huang, H., Shen, Y., and Soong, T.W. (2011b). Functional characterization of alternative splicing in the C-terminus of L-type CaV1.3 channels. Journal of Biological Chemistry.

Usukura, J., and Yamada, E. (1987). Ultrastructure of the synaptic ribbons in photoreceptor cells of Rana catesbeiana revealed by freeze-etching and freeze-substitution. Cell and Tissue Research 247, 483-488.

Uthaiah, R.C., and Hudspeth, A.J. (2010a). Molecular Anatomy of the Hair Cell's Ribbon Synapse. J. Neurosci. 30, 12387-12399.

Uthaiah, R.C., and Hudspeth, A.J. (2010b). Molecular Anatomy of the Hair Cell's Ribbon Synapse. J. Neurosci. 30, 12387-12399.

Winter, I.M., Robertson, D., and Yates, G.K. (1990). Diversity of characteristic frequency rate-intensity functions in guinea pig auditory nerve fibres. Hearing Research 45, 191-202.

Xu, W., and Lipscombe, D. (2001). Neuronal CaV1.3a1 L-Type Channels Activate at Relatively Hyperpolarized Membrane Potentials and Are Incompletely Inhibited by Dihydropyridines. J. Neurosci. 21, 5944-5951.

Yang, P.S., Alseikhan, B.A., Hiel, H., Grant, L., Mori, M.X., Yang, W., Fuchs, P.A., and Yue, D.T. (2006). Switching of $\mathrm{Ca} 2+-$ dependent inactivation of $\mathrm{Ca}(\mathrm{v}) 1.3$ channels by calcium binding proteins of auditory hair cells. J. Neurosci 26, 10677-10689.

Zampini, V., Johnson, S.L., Franz, C., Lawrence, N.D., Münkner, S., Engel, J., Knipper, M., Magistretti, J., Masetto, S., and Marcotti, W. (2010). Elementary properties of CaV1.3 Ca(2+) channels expressed in mouse cochlear inner hair cells. J. Physiol. (Lond.) 588, 187-199.

Zenisek, D., Davila, V., Wan, L., and Almers, W. (2003). Imaging calcium entry sites and ribbon structures in two presynaptic cells. J. Neurosci 23, 2538-2548.

Zheng, J., Shen, W., He, D.Z.., Long, K.B., Madison, L.D., and Dallos, P. (2000). Prestin is the motor protein of cochlear outer hair cells. Nature 405, 149-155. 


\section{List of Abbreviation}

AKAP79/150

AP

AZ

BAPTA

C.M.

C.V.

CaBP

CaMKII

CCD

CDF

CDI

CF

$\mathrm{C}_{\text {fast }}$

$\mathrm{C}_{\text {slow }}$

CtBP2

CTM

EGTA

EPSC

EPSP

FRET

FV

HEK

IHC

IV

$\mathrm{k}$

MET

myo6

$\mathrm{OHC}$

$\mathrm{P}_{\alpha}$
A-kinase anchoring protein

Action potential

Active zone

1,2-bis(o-aminophenoxy)ethane-N,N,N',N'-tetraacetic acid

Center of Mass

Coefficient of variation

calmodulin-like $\mathrm{Ca}^{2+}$-binding protein

$\mathrm{Ca}^{2+} /$ calmodulin-dependent protein kinase II

Charge-coupled device

$\mathrm{Ca}^{2+}$ dependent facilitation

$\mathrm{Ca}^{2+}$ dependent inactivation

characteristic frequency

fast-kinetic capacitance

slow-kinetic capacitance

C terminal binding protein 2

C-terminal modulator

ethylene glycol tetraacetic acid

evoked postsynaptic current

evoked postsynaptic potential

Fluorescence resonance energy transfer

Fluorescence-voltage relationship

Human Embryonic Kidney 293 cells

Inner hair cell

Current-voltage relationship

slope factor of voltage dependent activation

Mechanoelectrotransduction

Myosin VI

Outer hair cell

$\mathrm{p}$-value for hypothesis testing 
$\mathrm{Pr}_{\mathrm{r}}$

PSCM

RIM

rpm

$R_{\text {seal }}$

$R_{\text {series }}$

S.D.

SDCM

SGN

SK

SR

TAMRA

TEA

$\mathrm{V}_{0.5}$
Correlation Coefficient

Point scanning confocal microscopy

Rab3-interacting molecule

Rounds per minute

Seal resistance

Series resistance

Standard deviation

Spinning disk confocal microscopy

Spiral ganglion neuron

Slow-activated $\mathrm{Ca}^{2+}$ dependence $\mathrm{K}^{+}$channel

Spontaneous rate

Tetramethylrhodamine

Tetraethylammonium

Voltage for half activation 


\section{Acknowledgement}

I am grateful to have following people to accomplish this doctor thesis, without them, it could be a very difficult task.

I honestly thank for my supervisor, Dr. Tobias Moser. Thanks him to give me a chance to study here with this project. In these years, he usually came with good ideas, helpful discussion, encouragement, and was glad to provide much assistance on my experiments. I also need to thank some of my colleagues, Dr. Thomas Frank, who taught me how to initiate my PhD work and a lot of help about $\mathrm{Ca}^{2+}$ imaging and confocal system. Dr. Jakob Neef helped me to fix many problems of my patch-clamp setup and other electronic instruments. I also thank for the discussions with him about the ion channels and currents, they helped me a lot. I specially thank him for proofreading some part of this thesis. Thanks for Aaron Beson Wong and Dr. Mark Rutherford. They were enthusiastic at listen my findings, ridiculous ideas, and still provide me their opinions. I also thank for Dr. Fred Wolf and my colleague, Dr. Nikolai Chapochnikov. They provided me a lot of inspiring thinking and knowledge of mathematical and physical viewpoints. I specially thank for some technicians for assisting me to fix my complicated spinning disk setup. Mr. Joachim Niechoy from the workshop of university medical center, he has an excellent skill to produce every unique pieces for my setup and experiments. Mr. Mizuho Shimizu from Yokogawa company, he always responded to me immediately and gave me correct solutions when I met troubles of my spinning disk. Ms. Chun Xiao Bleau from Redshirt Imaging, she and her husband help me to fix problems of the CCD camera on line, and modified the firmware for me to accommodate my requirements.

Finally I want to thank for my wife, Linda. Thanks for her companion in these 4 years. My $\mathrm{PhD}$ career is not an easy time for her. Thanks for her to sacrifice her time on waiting and tolerating for my endless work. 


\section{Curriculum Vitae}

\section{Personal data}

Name:

Date of Birth:

Place of Birth:

Nationality:

Contact:
Tzu-Lun Wang

30.08.1982

Kaohsiung, Taiwan

Taiwan

Inner Ear Lab, Dept. of Otolaryngology,

University Medical Center of Göttingen,

Robert-Koch-Str. 40,

D-37075, Göttingen,

Germany

E-mail: twang@gwdg.de

TEL : +495513922843

\section{Education}

09.2001-06.2006

Bachelor Degree, Department of Zoology, National Taiwan University, Taipei, Taiwan

08.2006-07.2008 Master Degree, Institute of Zoology, National Taiwan University, Taipei, Taiwan

11.2008-present PhD studies, with Prof. Dr. Tobias Moser, University of Göttingen, Göttingen, Germany.

\section{Master Thesis}

"The Effects of Dynasore on Exocytosis and Endocytosis in Bovine Chromaffin Cells" Supervised by Dr. Chien-Yuan Pan

in the Institute of Zoology, National Taiwan University, Taipei, Taiwan

\section{Scholarship}

11.2009-10.2012

DAAD stipendium 